\title{
WEIGHTED NORM INEQUALITIES ON MORREY SPACES
}

\author{
SHOHEI NAKAMURA AND YOSHIHIRO SAWANO
}

\begin{abstract}
In this paper, we shall investigate weighted Morrey spaces by employing the sparse family. Particularly, we shall discuss the boundedness of the Hardy-Littlewood maximal operator and the singular integral operators on weighted Morrey spaces. In addition, we shall discuss the local sharp maximal inequalities on wighted Morrey spaces. Our results will cover Morrey spaces of Samko type and Komori-Shirai type.
\end{abstract}

\section{INTRODUCTION}

The Muckenhoupt class is sufficient for the Hardy-Littlewood maximal operator to be bounded on weighted Morrey spaces. In this paper, we will give better sufficient conditions on weights. In addition, we will observe that there exists an essential difference between the behavior of the Hardy-Littlewood maximal operator and the one of the singular integral operators on weighted Morrey spaces of Samko type; see Corollaries 1.19 and 1.20

First of all, we fix our notations. We denote the all Lebesgue measurable functions by $L^{0}\left(\mathbb{R}^{n}\right)$. By $\mathcal{Q}=\mathcal{Q}\left(\mathbb{R}^{n}\right)$, we mean the all cubes in $\mathbb{R}^{n}$ whose sides parallel to the coordinate axes. We denote the family of all dyadic cubes by $\mathcal{D}=\mathcal{D}\left(\mathbb{R}^{n}\right)$ and the family of all dyadic cubes with respect to $Q \in \mathcal{Q}$ by $\mathcal{D}(Q)$. A weight $w$ is a locally integrable function on $\mathbb{R}^{n}$ such that $w(x)>0$ for almost everywhere $x \in \mathbb{R}^{n}$. For weight $w$ and measurable set $E \subset \mathbb{R}^{n}$, we denote $w(E):=\int_{E} w(x) d x$. In addition, we denote a weighted measure by $d w$, that is, $d w(x)=w(x) d x$.

We define the weighted Morrey space $\mathcal{M}_{q}^{p}\left(w_{1}, w_{2}\right)$, where $0<q \leq p<\infty$ and $w_{1}, w_{2}$ are weights. The weighted Morrey space $\mathcal{M}_{q}^{p}\left(w_{1}, w_{2}\right)$ is the set of all functions $f \in L_{\text {loc }}^{q}\left(w_{2}\right)$ for which the quasi-norm

$$
\|f\|_{\mathcal{M}_{q}^{p}\left(w_{1}, w_{2}\right)}:=\sup _{Q \in \mathcal{Q}} w_{1}(Q)^{\frac{1}{p}-\frac{1}{q}}\left(\int_{Q}|f(x)|^{q} d w_{2}(x)\right)^{\frac{1}{q}}
$$

is finite. When $w_{1}=w_{2}=w, \mathcal{M}_{q}^{p}\left(w_{1}, w_{2}\right)$ corresponds to the weighted Morrey space $\mathcal{M}_{q}^{p}(w, w)$ introduced by Komori and Shirai in [23]. Meanwhile, when $w_{1}=d x$ and $w_{2}=w, \mathcal{M}_{q}^{p}\left(w_{1}, w_{2}\right)$ corresponds to the weighted Morrey space $\mathcal{M}_{q}^{p}(d x, w)$ introduced by Samiko in 33 .

In [30, we introduced the weight class $\mathcal{B}_{p, q}$ and the weighted integral condition in the context of the boundedness of the Hardy-Littlewood maximal operator on weighted Morrey spaces of Samko type.

Definition 1.1 ([30]). Let $0<q \leq p<\infty$ and $w$ be a weight.

2010 Mathematics Subject Classification. Primary 42B20; Secondary 42B25, 46E30.

Key words and phrases. Weighted Morrey spaces, Maximal operator, Singular integral operators, Local sharp maximal operator. 
(1) One says that a weight $w$ is in the class $\mathcal{B}_{p, q}$ if there exists $C_{p, q}>0$ such that for any $Q_{0} \in \mathcal{Q}$,

$$
\sup _{Q \in \mathcal{Q}: Q \subset Q_{0}} \Phi_{p, q, w}(Q) \leq C_{p, q} \Phi_{p, q, w}\left(Q_{0}\right)
$$

or equivalently,

$$
\left\|\chi_{Q_{0}}\right\|_{\mathcal{M}_{q}^{p}(d x, w)} \sim \Phi_{p, q, w}\left(Q_{0}\right)
$$

hold, where we defined

$$
\Phi_{p, q, w}(Q):=|Q|^{\frac{1}{p}}\left(\frac{w(Q)}{|Q|}\right)^{\frac{1}{q}} \quad(Q \in \mathcal{Q}) .
$$

(2) The weighted integral condition for $p, q$ and $w$ holds, if there exists a constant $C>0$ such that

$$
\int_{1}^{\infty} \frac{1}{\Phi_{p, q, w}(s Q)} \frac{d s}{s} \leq \frac{C}{\Phi_{p, q, w}(Q)} \quad(Q \in \mathcal{Q})
$$

holds.

Note that the weighted integral condition (2) for $p, q$ and $w$ implies $w \in \mathcal{B}_{p, q}$; see [30] for details. Using these two notions, we aim to give the sufficiently conditions of the boundedness of the Hardy-Littlewood maximal operator and singular integral operator. To this end, we first recall the definition of the Hardy-Littlewood maximal operator. By $M$, we mean the (unweighted) Hardy-Littlewood maximal operator:

$$
M f(x):=\sup _{Q \in \mathcal{D}} \frac{1}{|Q|} \int_{Q}|f(y)| d y \cdot \chi_{Q}(x) .
$$

Recall the boundedness results of the Hardy-Littlewood maximal operator on weighted Morrey spaces. To this end, we first recall the Muckenhoupt class $A_{q}$. For $1<q<\infty$, a weight $w$ belongs to the class $A_{q}$ if

$$
[w]_{A_{q}}:=\sup _{Q \in \mathcal{Q}}\left(\frac{w(Q)}{|Q|}\right)\left(\frac{1}{|Q|} \int_{Q} w(x)^{-\frac{1}{q-1}} d x\right)^{q-1}<\infty .
$$

For the case of $q=1$, we define

$$
[w]_{A_{1}}:=\operatorname{esssup}_{x \in \mathbb{R}^{n}} \frac{M w(x)}{w(x)},
$$

and denote the set of all weights $w$ for which $[w]_{A_{1}}$ is finite by $A_{1}$. Moreover, we set $A_{\infty}:=$ $\bigcup_{q \in[1, \infty)} A_{q}$.

Here we give a sufficient conditions for the boundedness of $M$ on weighted Morrey spaces.

Theorem 1.2. Let $1<q \leq p<\infty$ and $w \in A_{q}$.

(1) (23] We have that

$$
\|M f\|_{\mathcal{M}_{q}^{p}(w, w)} \lesssim_{p, q, w}\|f\|_{\mathcal{M}_{q}^{p}(w, w)}, \quad\left(f \in \mathcal{M}_{q}^{p}(w, w)\right) .
$$

(2) (30]) In addition, we assume $w \in \mathcal{B}_{p, q}$. Then we have that

$$
\|M f\|_{\mathcal{M}_{q}^{p}(d x, w)} \lesssim p, q, w\|f\|_{\mathcal{M}_{q}^{p}(d x, w)}, \quad\left(f \in \mathcal{M}_{q}^{p}(d x, w)\right) .
$$

Now, let us state our first main theorem: the necessary conditions for the boundedness of these two operators. Note that we say that the weight $w$ satisfies the doubling condition if there exists a constant $C>0$ such that for any $Q \in \mathcal{Q}, w(2 Q) \leq C w(Q)$ holds. 
Theorem 1.3. Let $1<q \leq p<\infty$ and $w$ be a weight. Assume that $M$ is bounded on $\mathcal{M}_{q}^{p}(d x, w)$. Then we have the following:

(1) $w \in \mathcal{B}_{p, q} \cap A_{q+1}$.

(2) $w^{-\frac{1}{q-1}} \in \mathcal{B}_{p, q}$ implies $w \in A_{q}$.

(3) $w^{-\frac{1}{q-1}} \in A_{\infty}$ is equivalent to $w \in A_{q}$.

Recently, Tanaka characterized the boundedness of $M$ on $\mathcal{M}_{q}^{p}\left(d x, w_{\alpha}\right)$ with the power weight $w_{\alpha}(x):=|x|^{\alpha}$ in his nice paper [41, Proposition 4.2]. His works mainly depend on the results obtained by Adams and Xiao in [1. To obtain the characterization, he focused on the dual equation of Morrey spaces. Let us recall the Köthe dual spaces of Morrey spaces and overview his works. Let $0<\alpha \leq n$ and $E \subset \mathbb{R}^{n}$. Then the $\alpha$-dimensional Hausdorff content of $E$ is defined by

$$
H^{\alpha}(E):=\inf \left\{\sum_{j} \ell\left(Q_{j}\right)^{\alpha}:\left\{Q_{j}\right\}_{j} \subset \mathcal{Q}, \bigcup_{j} Q_{j} \supset E\right\} .
$$

For a non-negative function $\phi \geq 0$, the Choquet integral of $\phi$ with respect to the Hausdorff content $H^{\alpha}$ is defined by

$$
\int_{\mathbb{R}^{n}} \phi d H^{\alpha}:=\int_{0}^{\infty} H^{\alpha}\left(\left\{x \in \mathbb{R}^{n}: \phi(x)>t\right\}\right) d t .
$$

Note that by the definition, it follows that

$$
\int_{\mathbb{R}^{n}}|f(x)|^{q} \phi(x) d x \leq\|f\|_{\mathcal{M}_{q}^{p}(d x, d x)}^{q} \int_{\mathbb{R}^{n}} \phi d H^{n(1-q / p)} .
$$

See [1] or [41, (2.3)] for the detail.

Definition 1.4 ([1]). Define $\mathfrak{B}_{\alpha}:=\left\{b \in A_{1}: \int_{\mathbb{R}^{n}} b d H^{\alpha} \leq 1\right\}$ for $0<\alpha<n$.

A typical example of $\mathfrak{B}_{\alpha}$ is

$$
b_{Q}:=\frac{\left(M \chi_{Q}\right)^{\alpha / n+\varepsilon}}{\ell(Q)^{\alpha}}
$$

where $Q \in \mathcal{Q}$ and $\varepsilon \in\left(0,1-\frac{\alpha}{n}\right)$ are arbitrary. Then for $1<q \leq p<\infty$, the space $H^{q^{\prime}, n(1-q / p)}(d x, d x)=H^{q^{\prime}, n(1-q / p)}$ is defined by the set of all measurable functions $f$ for which norm

$$
\|f\|_{H^{q^{\prime}, n(1-q / p)}}:=\inf _{b \in \mathfrak{B}_{n(1-q / p)}}\left(\int_{\mathbb{R}^{n}}|f(x)|^{q^{\prime}} b(x)^{-\frac{q^{\prime}}{q}} d x\right)^{\frac{1}{q^{\prime}}}
$$

is finite.

The following are proved in [1] or [41, (2.5)]: For $1<q \leq p<\infty$, we have

$$
\left|\int_{\mathbb{R}^{n}} f(x) g(x) d x\right| \leq C\|f\|_{\mathcal{M}_{q}^{p}(d x, d x)}\|g\|_{H^{q^{\prime}, n(1-q / p)}},
$$

and that

$$
\|g\|_{H^{q^{\prime}, n(1-q / p)}} \sim \sup \left\{\|f \cdot g\|_{L^{1}}: f \in \mathcal{M}_{q}^{p}(d x, d x):\|f\|_{\mathcal{M}_{q}^{p}(d x, d x)} \leq 1\right\},
$$

for any measurable function $g$.

In this paper, we denote all weights such that the Hardy-Littlewood maximal operator $M$ is bounded on $\mathcal{M}_{q}^{p}(d x, w)$ by $H L M_{q}^{p}$, namely,

$$
H L M_{q}^{p}:=\left\{w: \text { weight, } M: \mathcal{M}_{q}^{p}(d x, w) \rightarrow \mathcal{M}_{q}^{p}(d x, w)\right\} .
$$


Theorem 1.5. 41, Thoerem 3.1] Let $1<q \leq p<\infty$ and $w$ be a weight. Consider the following four statements:

(1) $w$ is in the class $H L M_{q}^{p}$.

(2) For any cube $Q \in \mathcal{Q}$, we have

$$
\frac{1}{|Q|}\left\|w^{\frac{1}{q}} \chi_{Q}\right\|_{\mathcal{M}_{q}^{p}(d x, d x)}\left\|w^{-\frac{1}{q}} \chi_{Q}\right\|_{H^{q^{\prime}, n(1-q / p)}} \lesssim 1 .
$$

(3) For any $Q_{0} \in \mathcal{Q}$, we have

$$
\inf _{b \in \mathfrak{B}_{n(1-q / p)}}\left(\sup _{\substack{Q \in \mathcal{Q}: \\ Q \subset Q_{0}}} \frac{1}{\sigma(Q)} \int_{Q} M\left[\sigma \chi_{Q}\right](x)^{q} w(x) d x\right) \lesssim \ell\left(Q_{0}\right)^{n(1-q / p)},
$$

where $\sigma:=(b w)^{-\frac{q^{\prime}}{q}}$.

(4) There exists a constant $a>1$ such that for any $Q_{0} \in \mathcal{Q}$, we have

$$
\inf _{b \in \mathfrak{B}_{n(1-q / p)}}\left(\sup _{\substack{Q \in \mathcal{Q}: \\ Q \subset Q_{0}}} \frac{w(Q)}{|Q|}\left(\frac{1}{|Q|} \int_{Q}[b(x) w(x)]^{-\frac{a q^{\prime}}{q}} d x\right)^{\frac{q}{a q^{\prime}}}\right) \lesssim \ell\left(Q_{0}\right)^{n(1-q / p)} .
$$

Then it holds that

- 1 implies 2 .

- 2 and 3 imply 1 .

- 2 and 4 imply 1 .

The condition (77) is natural in view of the dual inequality (57). In addition, the condition (17) corresponds to the $A_{p}$ condition in the case of Lebesgue setting: $p=q$. However, we need additional conditions (8) or (9) for the boundedness of $M$ in Theorem 1.5. This is a serious problem. When $w(x)=w_{\alpha}(x):=|x|^{\alpha}$, as is shown in [11, Proposition 4.2], the necessary condition (7) is equivalent to

$$
-\frac{q}{p} n \leq \alpha<n\left(q-\frac{q}{p}\right)
$$

which implies the sufficient condition (9). That is, $M$ is bounded on $\mathcal{M}_{q}^{p}\left(d x, w_{\alpha}\right)$ if and only if $\alpha$ satisfies (10).

Let us consider what can we say from the condition (7). Since we may assume $w \in \mathcal{B}_{p, q}$ when we consider the boundedness of $M$ by Theorem 1.3, the condition (7) turns into

$$
\frac{1}{\left|Q_{0}\right|}\left|Q_{0}\right|^{\frac{1}{p}}\left(\frac{w\left(Q_{0}\right)}{\left|Q_{0}\right|}\right)^{\frac{1}{q}}\left\|w^{-\frac{1}{q}} \chi_{Q_{0}}\right\|_{H^{q^{\prime}, n(1-q / p)}} \leq C_{0} .
$$

Moreover, if we recall the definition of $H^{q^{\prime}, n(1-q / p)}$, then the condition (7) yields that for any $Q_{0} \in \mathcal{Q}$, there exists $b_{Q_{0}} \in \mathfrak{B}_{n(1-q / p)}$ such that

$$
\frac{1}{\left|Q_{0}\right|} \Phi_{p, q, w}\left(Q_{0}\right)^{q}\left(\frac{1}{\left|Q_{0}\right|} \int_{Q_{0}}\left[b_{Q_{0}}(x) w(x)\right]^{-\frac{1}{q-1}} d x\right)^{q-1} \leq C_{0}^{q} .
$$

The equation (11) seems to be a $A_{q}$ condition, but the difficulty is that the equation (11) holds for only $Q_{0}$. That is, there are no information of $b_{Q_{0}}$ on $Q \in \mathcal{D}\left(Q_{0}\right)$. Motivated by such situation, by imposing additional assumption for $b_{Q_{0}}$, we obtain another sufficient condition for 
the weak type boundedness of $M$. This is our next main theorem. The weighted weak Morrey space of Samko type $w \mathcal{M}_{q}^{p}(d x, w)$ is the set of all $f \in L_{\mathrm{loc}}^{q}(w)$ for which

$$
\|f\|_{w \mathcal{M}_{q}^{p}(d x, w)}:=\sup _{Q \in \mathcal{D}} \sup _{t>0}|Q|^{\frac{1}{p}-\frac{1}{q}} t w(\{x \in Q:|f(x)|>t\})^{\frac{1}{q}}
$$

is finite.

Theorem 1.6. Let $1<q \leq p<\infty$ and $w \in \mathcal{B}_{p, q}$. Assume that for any $Q_{0} \in \mathcal{D}$, there exists $b_{Q_{0}} \in \mathfrak{B}_{n(1-q / p)}$ such that

$$
\frac{1}{|Q|} \Phi_{p, q, w}(Q)^{q}\left(\frac{1}{|Q|} \int_{Q}\left[b_{Q_{0}}(x) w(x)\right]^{-\frac{1}{q-1}} d x\right)^{q-1} \leq C\left(\frac{\left|Q_{0}\right|}{|Q|}\right)^{1-\frac{q}{p}}
$$

holds for all $Q \in \mathcal{D}\left(Q_{0}\right)$. Then we have

$$
\|M f\|_{w \mathcal{M}_{q}^{p}(d x, w)} \leq C\|f\|_{\mathcal{M}_{q}^{p}(d x, w)}, \quad\left(f \in \mathcal{M}_{q}^{p}(d x, w)\right) .
$$

Two important remarks may be in order.

\section{Remark 1.7.}

(1) If one assumes that $w \in A_{q} \cap \mathcal{B}_{p, q}$, then one can construct $b_{Q_{0}} \in \mathfrak{B}_{n(1-q / p)}$ satisfying (12) in Theorem 1.6. In fact, one has only to take

$$
b_{Q_{0}}(x)=\frac{\left(M \chi_{Q_{0}}(x)\right)^{1-\frac{q}{p}+\varepsilon}}{\left|Q_{0}\right|^{1-\frac{q}{p}}}
$$

as in (4). Hence, Theorem 11 improves Theorem 1.2 in the case of weak type boundedness. Moreover, if $w$ is in $A_{q} \cap \mathcal{B}_{p, q}$, then $w$ satisfies the sufficient conditions (8) and (9) in Theorem [1.5.

(2) In particular, in the case of $w=w_{\alpha}$ with (10), one can find the $b_{Q_{0}} \in \mathfrak{B}_{n(1-q / p)}$ satisfying (12); see Lemma 3.1. Hence, one can reprove the weak type boundedness of $M$ on $\mathcal{M}_{q}^{p}\left(d x, w_{\alpha}\right)$ in an optimal range (10).

By virtue of the additional assumption (12), the assumption in Theorem 1.6 is stronger than (77). Hence, the problem of the characterization of the boundedness of $M$ on $\mathcal{M}_{q}^{p}(d x, w)$ for a general weight is still open.

Next our main theorem deals with the local sharp maximal inequalities on the weighted Morrey spaces. We recall the local sharp maximal operator introduced in [19, 42].

Definition $1.8([13,26])$. Let $f \in L^{0}\left(\mathbb{R}^{n}\right)$ and $Q \in \mathcal{Q}$.

(1) The decreasing rearrangement of $f$ on $\mathbb{R}^{n}$ is defined by

$$
f^{*}(t):=\left\{\rho>0:\left|\left\{x \in \mathbb{R}^{n}:|f(x)|>\rho\right\}\right|<t\right\}, \quad(0<t<\infty) .
$$

(2) The local mean oscillation of $f$ on $Q$ is defined by

$$
\omega_{\lambda}(f ; Q):=\inf _{c \in \mathbb{C}}\left((f-c) \chi_{Q}\right)^{*}(\lambda|Q|), \quad\left(\lambda \in\left(0,2^{-1}\right)\right) .
$$

(3) Assume that the function $f$ is real-valued. The median of $f$ over $Q$ denoted by $m_{f}(Q)$ is a real number satisfying that

$$
\left|\left\{x \in Q: f(x)>m_{f}(Q)\right\}\right|, \quad\left|\left\{x \in Q: f(x)<m_{f}(Q)\right\}\right| \leq \frac{1}{2} .
$$

Note that the median $m_{f}(Q)$ is possibly non-unique. 
For $\lambda \in\left(0,2^{-1}\right)$ and $Q_{0} \in \mathcal{Q}$, the dyadic local sharp maximal operator $M_{\lambda ; Q_{0}}^{\sharp, d}$ is defined by

$$
M_{\lambda ; Q_{0}}^{\sharp, d} f(x):=\sup _{Q \in \mathcal{D}\left(Q_{0}\right)} \omega_{\lambda}(f ; Q) \chi_{Q}(x), \quad\left(x \in \mathbb{R}^{n}, f \in L^{0}\left(\mathbb{R}^{n}\right)\right) .
$$

In this paper, we are interested in the following sharp maximal operator:

$$
M_{\lambda}^{\sharp, d} f(x):=\sup _{Q_{0} \in \mathcal{Q}} \sup _{Q \in \mathcal{D}\left(Q_{0}\right)} \omega_{\lambda}(f ; Q) \chi_{Q}(x) \quad\left(x \in \mathbb{R}^{n}, f \in L^{0}\left(\mathbb{R}^{n}\right)\right) .
$$

As is well known, the Fefferman-Stein sharp maximal operator defined by

$$
f^{\sharp, \eta}(x):=\sup _{Q \in \mathcal{Q}}\left(\frac{1}{|Q|} \int_{Q}\left|f(x)-f_{Q}\right|^{\eta} d x\right)^{\frac{1}{\eta}},
$$

where $\eta>0$ and $f_{Q}:=\frac{1}{|Q|} \int_{Q} f(x) d x$, is a useful tool to estimate the oscillation of the function $f$. When $\eta=1$, we abbreviate $f^{\sharp, 1}$ to $f^{\sharp}$. There exists a close relation between these two types of the sharp maximal operators provided by Jawerth and Torchinsky in [18]:

$$
M^{(\eta)} M_{\lambda}^{\sharp, d} f(x) \sim_{\eta, \lambda} f^{\sharp, \eta}(x),
$$

for sufficiently small $\lambda$, where $M^{(\eta)}$ denotes the powered Hardy-Littlewood maximal operator defined by

$$
M^{(\eta)} f(x):=\sup _{Q \in \mathcal{Q}}\left(\frac{1}{|Q|} \int_{Q}|f(x)|^{\eta} d x\right)^{\frac{1}{\eta}} \chi_{Q}(x) .
$$

When $\eta=1, M^{(1)}$ is the original Hardy-Littlewood maximal operator.

In [26], Lerner proved the following theorem:

Theorem $1.9([26])$. Let $f \in L^{0}\left(\mathbb{R}^{n}\right)$ and $Q_{0} \in \mathcal{Q}$. Then there exists a sparse family of $\left\{Q_{j}^{k}\right\}_{k \in \mathbb{N}_{0}, j \in J_{k}} \subset \mathcal{D}\left(Q_{0}\right)$ such that for a.e. $x \in Q_{0}$,

$$
\left|f(x)-m_{f}\left(Q_{0}\right)\right| \leq 4 M_{\lambda_{n} ; Q_{0}}^{\sharp, d} f(x)+2 \sum_{k \in \mathbb{N}_{0}} \sum_{j \in J_{k}} \omega_{\lambda_{n}}\left(f ; Q_{j}^{k}\right) \chi_{Q_{j}^{k}}(x) .
$$

Here, $\lambda_{n}:=2^{-n-2}$.

This theorem is a starting point of the next topic. Here, we say that the family $\left\{Q_{j}^{k}\right\}_{k \in \mathbb{N}_{0}, j \in J_{k}}$ is a sparse family if the following properties hold:

(1) for each fixed $k \in \mathbb{N}_{0}$, the cubes $\left\{Q_{j}^{k}\right\}_{j \in J_{k}}$ are disjoint;

(2) if $\Omega_{k}:=\bigcup_{j \in J_{k}} Q_{j}^{k}$, then $\Omega_{k+1} \subset \Omega_{k}$;

(3) $\left|\Omega_{k+1} \cap Q_{j}^{k}\right| \leq \frac{1}{2}\left|Q_{j}^{k}\right|$ for all $j \in J_{k}$.

Now, we state our next main theorem in this paper. Throughout this paper, for $w \in A_{\infty}$, we fix the parameter $\lambda_{w}^{\prime}$ determined by $w$ so that $\lambda_{w}^{\prime}<2^{-1-2^{n+3}[w]_{A_{\infty}}}$ and let $\lambda_{w}:=2^{-n-2} \lambda_{w}^{\prime}$, where

$$
[w]_{A_{\infty}}:=\lim _{r \downarrow 0}[w]_{A_{r}} .
$$

Theorem 1.10. Let $0<s \leq q \leq p<\infty$ and $w \in A_{\infty}$.

(1) (Komori-Shirai type) For all $f \in L^{0}\left(\mathbb{R}^{n}\right)$, it holds that

$$
\|f\|_{\mathcal{M}_{q}^{p}(w, w)} \sim_{p, s, q, w}\left\|M_{\lambda_{w}}^{\sharp, d} f\right\|_{\mathcal{M}_{q}^{p}(w, w)}+\|f\|_{\mathcal{M}_{s}^{p}(w, w)} .
$$


(2) (Samko type) Additionally, we assume $w \in \mathcal{B}_{p, q}$. Then for all $f \in L^{0}\left(\mathbb{R}^{n}\right)$, it holds that

$$
\begin{aligned}
\|f\|_{\mathcal{M}_{q}^{p}(d x, w)} \quad \sim_{p, s, q, w} & \left\|M_{\lambda_{w}}^{\sharp, d} f\right\|_{\mathcal{M}_{q}^{p}(d x, w)} \\
& +\sup _{Q \in \mathcal{Q}} \Phi_{p, q, w}(Q)\left(\frac{1}{w(Q)} \int_{Q}|f(x)|^{s} d w(x)\right)^{\frac{1}{s}} .
\end{aligned}
$$

The last term in the right-hand side of (14) is not so artificial in view of [36, (44)]. By the Hölder inequality, it is easy to see

$$
\sup _{Q \in \mathcal{Q}} \Phi_{p, q, w}(Q)\left(\frac{1}{w(Q)} \int_{Q}|f(x)|^{s} d w(x)\right)^{\frac{1}{s}} \leq\|f\|_{\mathcal{M}_{q}^{p}(d x, w)}
$$

for all $f \in L^{0}\left(\mathbb{R}^{n}\right)$. This corresponds to the relation:

$$
\|f\|_{\mathcal{M}_{s}^{p}(w, w)} \leq\|f\|_{\mathcal{M}_{q}^{p}(w, w)}
$$

for all $f \in L^{0}\left(\mathbb{R}^{n}\right)$.

In the original sharp maximal inequality, we need to assume some integrability for $f$; see Theorem 4.7 for example. However, in Theorem 1.10, we can apply the sharp maximal inequality for any measurable functions. Note that the same refinement for the unweighted case (but the inhomogeneous setting) is obtained in [37, Theorem 1.3]; see Theorem 4.6 in the present paper for the precise formulation.

If we impose a suitable condition for $f$, then we can recover the original version of the sharp maximal inequality, see Corollaries 1.13 and 4.5 as well.

Theorem 1.11. Let $0<s \leq q \leq p<\infty, w \in A_{\infty}$.

(1) (Komori-Shirai type) Assume that $f \in L^{0}\left(\mathbb{R}^{n}\right)$ satisfies

$$
m_{f}\left(2^{l} Q\right) \rightarrow 0
$$

as $l \rightarrow \infty$ for any $Q \in \mathcal{Q}$ and for some medians $\left\{m_{f}\left(2^{l} Q\right)\right\}_{l \in \mathbb{N}_{0}}$. Then we have that

$$
\|f\|_{\mathcal{M}_{s}^{p}(w, w)} \lesssim_{p, s, w}\left\|M_{\lambda_{w}}^{\sharp, d} f\right\|_{\mathcal{M}_{s}^{p}(w, w)} \leq\left\|M_{\lambda_{w}}^{\sharp, d} f\right\|_{\mathcal{M}_{q}^{p}(w, w)} .
$$

(2) (Samko type) We assume the weighted integral condition (2) for $p, q$ and $w$. Then for any $f \in L^{0}\left(\mathbb{R}^{n}\right)$ satisfying (17) for any $Q \in \mathcal{Q}$ and for some medians $\left\{m_{f}\left(2^{l} Q\right)\right\}_{l \in \mathbb{N}}$, we have that

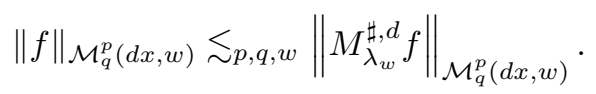

Remark 1.12. In the case of the weighted Morrey space $\mathcal{M}_{q}^{p}(d x, w)$ of Samko type, the weighted integral condition (2) for $p, q$, and $w$ and (17) are natural in view of the following example. In fact, if we take $0<q<p<\infty, f_{0} \equiv 1$ and $w_{0}(x):=|x|^{-\frac{q}{p} n} \in A_{\infty} \cap \mathcal{B}_{p, q}$, then we know that $M f_{0} \equiv 1, f_{0}^{\sharp}=0$ and $0<\left\|M f_{0}\right\|_{\mathcal{M}_{q}^{p}\left(d x, w_{0}\right)}=\left\||x|^{-\frac{n}{p}}\right\|_{\mathcal{M}_{q}^{p}(d x, d x)}<\infty$, which implies that the inequality

$$
\|f\|_{\mathcal{M}_{q}^{p}\left(d x, w_{0}\right)} \lesssim\left\|f^{\sharp}\right\|_{\mathcal{M}_{q}^{p}\left(d x, w_{0}\right)}
$$

fails even when $f$ satisfies $M f \in \mathcal{M}_{q}^{p}\left(d x, w_{0}\right)$. One can notice that for $w_{0}(x)=|x|^{-\frac{q}{p} n}$, the weighted integral condition (2) for $p, q$ and $w_{0}$ fails to hold; see [30.

We note that for $0<s \leq q \leq p<\infty$,

$$
\sup _{Q \in \mathcal{Q}} \Phi_{p, q, w}(Q)\left(\frac{1}{w(Q)} \int_{Q}|f(x)|^{s} d w(x)\right)^{\frac{1}{s}} \leq\|f\|_{\mathcal{M}_{q}^{p}(d x, w)}
$$

holds by Hölder's inequality. Hence, combining Theorems 1.10 and 1.11, we obtain the following: 
SHOHEI NAKAMURA AND YOSHIHIRO SAWANO

Corollary 1.13. Let $0<q \leq p<\infty, w \in A_{\infty}$. Assume that $f \in L^{0}\left(\mathbb{R}^{n}\right)$ satisfies (17) for any $Q \in \mathcal{Q}$ and for some medians $\left\{m_{f}\left(2^{l} Q\right)\right\}_{l \in \mathbb{N}_{0}}$. Then

$$
\|f\|_{\mathcal{M}_{q}^{p}(w, w)} \sim_{p, q, w}\left\|M_{\lambda_{w}}^{\sharp, d} f\right\|_{\mathcal{M}_{q}^{p}(w, w)} .
$$

Moreover, if the weighted integral condition (2) for $p, q$ and $w$ holds, then we have that

$$
\|f\|_{\mathcal{M}_{q}^{p}(d x, w)} \sim_{p, q, w}\left\|M_{\lambda_{w}}^{\sharp, d} f\right\|_{\mathcal{M}_{q}^{p}(d x, w)} .
$$

Finally, we apply Theorem 1.11 to obtain the the boundedness of the singular integral operators and commutators on $\mathcal{M}_{q}^{p}(d x, w)$. First, we recall the definitions of the singular integral operators and commutators together with their recent results. A singular integral operator $T$ is a bounded linear operator on the unweighted $L^{2}$ space, for which there exists a kernel $K$ on $\mathbb{R}^{n} \times \mathbb{R}^{n}$ satisfying the following conditions:

(1) (Size condition) There exists $C>0$ such that $|K(x, y)| \leq \frac{C}{|x-y|^{n}}$ for any $x \neq y$.

(2) (Hörmander condition) For some $\theta \in(0,1]$,

$$
|K(x+h, y)-K(x, y)|+|K(x, y+h)-K(x, y)| \leq \frac{C|h|^{\theta}}{|x-y|^{n+\theta}}, \quad \text { if }|x-y|>2|h| .
$$

(3) If $f \in L_{\mathrm{c}}^{\infty}$, the set of all compactly supported $L^{\infty}$-functions, then

$$
T f(x)=\int_{\mathbb{R}^{n}} K(x, y) f(y) d y \quad(x \notin \operatorname{supp}(f)) .
$$

As Coifman and Fefferman showed in [4, Theorems I and III], $T$ is well defined as a bounded operator on $L^{q}(w)$, when $w \in A_{q}$ with $1<q<\infty$.

The commutator of a singular integral operator $T$ with a locally integrable function $b$ is initially defined by

$$
[b, T] f:=b \cdot T f-T(b \cdot f),
$$

for $f \in L_{\mathrm{c}}^{\infty}$. A locally integrable function $b$ is said to be a BMO function, if

$$
\|b\|_{\mathrm{BMO}}:=\left\|b^{\sharp}\right\|_{L^{\infty}}<\infty \text {. }
$$

When $b$ is a BMO function, we can extend the commutator $[b, T]$ as a bounded linear operator on $L^{p}\left(\mathbb{R}^{n}\right)$ for all $1<p<\infty$; see [10, Theorem 3.5.6] for example. We also note that in the endpoint case: $p=1$, the commutators are more singular than the singular integral operators. We refer [35] for the result of the commutator in the endpoint case: $p=1$.

In [30, we proved the following:

Theorem 1.14 (30]). Let $1<q \leq p<\infty$ and $w \in A_{q}$ satisfy the weighted integral condition (2) for $p, q$ and $w$. Then we can extend the singular integral operator $T$ to a bounded linear operator on $\mathcal{M}_{q}^{p}(d x, w)$ :

$$
\|T f\|_{\mathcal{M}_{q}^{p}(d x, w)} \leq C\|f\|_{\mathcal{M}_{q}^{p}(d x, w)}, \quad\left(f \in \mathcal{M}_{q}^{p}(d x, w)\right) .
$$

In particular, if we recall that the weighted integral condition (2) for $p, q$ and $w_{\alpha}$ is equivalent to $\alpha>-\frac{q}{p} n$; see [30, then the sufficient condition in Theorem 1.14 turns into

$$
-\frac{q}{p} n<\alpha<n(q-1)
$$

in the case of power weights $w_{\alpha}$. Meanwhile, in 33, Samko obtained the characterization of the boundedness of the Hilbert transform in the case of power weights. The Hilbert transform 
$H$ is a singular integral operator defined by

$$
H f(x):=\lim _{\varepsilon \rightarrow 0} \int_{\{y \in \mathbb{R}:|x-y|>\varepsilon\}} \frac{f(y)}{x-y} d y \quad\left(f \in L^{2}(\mathbb{R})\right) .
$$

Theorem 1.15 (33]). Let $1<q \leq p<\infty$ and $\alpha>-1$. The Hilbert transform $H$ is bounded on $\mathcal{M}_{q}^{p}\left(d x, w_{\alpha}\right)$, if and only if

$$
-\frac{q}{p}<\alpha<q-\frac{q}{p}
$$

By comparing (20) and (21), we see that the $A_{q}$ condition is too strong in the frame work of $\mathcal{M}_{q}^{p}(d x, w)$. In addition, we see that by comparing Theorems 1.5 with (10) and Theorem 1.15 . a gap exists between the boundedness of the Hardy-Littlewood maximal operator and the one of the singular integral operators: $\alpha=-\frac{q}{p} n$. We will observe that such phenomenon comes from the weighted integral condition (2) for $p, q$ and $w$ in Corollaries 1.19 and 1.20.

Before we state our next theorem related to the boundedness of $T$ on $\mathcal{M}_{q}^{p}(d x, w)$, we recall some examples of the singular integral operators.

Definition 1.16 ([3]). A singular integral operator $T$ is called a genuine singular integral operator if there exist constants $C, \theta>0$ and $R \in O(n)$ such that for any $x, y \in \mathbb{R}^{n}$ satisfying $x-y \in V_{\theta, R}:=R\left\{u=\left(u^{\prime}, u_{n}\right) \in \mathbb{R}^{n}:\left|u^{\prime}\right|<\theta\left|u_{n}\right|\right\}$, the integral kernel $K$ satisfies

$$
K(x, y) \geq \frac{C}{|x-y|^{n}}
$$

where $O(n)$ denotes the set of all orthogonal matrices in $\mathbb{R}^{n}$.

A typical example of the genuine singular integral operator is the Riesz transform defined by

for $i=1, \ldots, n$.

$$
R_{i} f(x):=\lim _{\varepsilon \rightarrow 0} \int_{\left\{y \in \mathbb{R}^{n}:\left|x_{i}-y_{i}\right|>\varepsilon\right\}} \frac{x_{i}-y_{i}}{|x-y|^{n+1}} f(y) d y \quad\left(f \in L^{2}\left(\mathbb{R}^{n}\right)\right),
$$

Now, we state the boundedness result of the singular integral operators.

Theorem 1.17. Let $1<q \leq p<\infty$ and $w$ be a weight.

(1) Assume that $w \in H L M_{q}^{p}$ satisfies the weighted integral condition (2) for $p, q$ and $w$. Then we can extend any singular integral operator $T_{0}$ initially defined on $L_{\mathrm{c}}^{\infty}$ to a bounded linear operator $T$ on $\mathcal{M}_{q}^{p}(d x, w)$ :

$$
\|T f\|_{\mathcal{M}_{q}^{p}(d x, w)} \leq C\|f\|_{\mathcal{M}_{q}^{p}(d x, w)} \quad\left(f \in \mathcal{M}_{q}^{p}(d x, w)\right) .
$$

(2) As a converse assertion, we have the following:

(a) If the Riesz transform $R_{i}$ is bounded on $\mathcal{M}_{q}^{p}(d x, w)$ for some $i=1, \ldots, n$, then $w$ satisfies (7).

(b) Assume that $w \in \mathcal{B}_{p, q}$ satisfies the doubling condition. If there exists a genuine singular integral operator $T$ which is bounded on $\mathcal{M}_{q}^{p}(d x, w)$, then the weighted integral condition (2) for $p, q$ and $w$ holds.

Remark 1.18. As in Remark 1.7, one already knows that $A_{q} \cap \mathcal{B}_{p, q} \subset H L M_{q}^{p}$. Hence, Theorem 1.17 improves Theorem 1.14 .

Moreover, once we obtain (7), then it follows that $w$ is in $\mathcal{B}_{p, q}$ and satisfies the doubling condition; see Lemma 2.2. Hence, we have the following:

Corollary 1.19. Let $1<q \leq p<\infty$ and $w$ be a weight. 
(1) Assume that $w$ is in $H L M_{q}^{p}$ and satisfies the weighted integral condition (2) for $p, q$ and $w$. Then for any $i=1, \ldots, n$, the Riesz transform $R_{i}$ is bounded on $\mathcal{M}_{q}^{p}(d x, w)$.

(2) Conversely, if we assume that the Riesz transform $R_{i}$ is bounded on $\mathcal{M}_{q}^{p}(d x, w)$ for some $i=1, \ldots, n$, then $w$ satisfies (7) and the weighted integral condition (2) for $p, q$ and $w$.

Since there exists a gap between the conditions $w \in H L M_{q}^{p}$ and (7), Corollary 1.19 does not completely characterize the boundedness of $R_{i}$. However, when we focus on the power weight $w_{\alpha}$, we already know that $w_{\alpha} \in H L M_{q}^{p}$ is equivalent to (7) or equivalently, (10); see [41, Proposition 4.2]. Hence, we can characterize the boundedness of $R_{i}$ on $\mathcal{M}_{q}^{p}\left(d x, w_{\alpha}\right)$. We also recall that the weighted integral condition (2) for $p, q$ and $w_{\alpha}$ is equivalent to $\alpha>-\frac{q}{p} n$.

Corollary 1.20. Let $1<q \leq p<\infty$ and $\alpha>-n$. The Riesz transform $R_{i}, i=1, \ldots, n$ is bounded on $\mathcal{M}_{q}^{p}\left(d x, w_{\alpha}\right)$ if and only if

$$
-\frac{q}{p} n<\alpha<n\left(q-\frac{q}{p}\right) .
$$

Note that Corollary 1.20 contains Theorem 1.15 as a special case.

Finally, we obtain a sufficient condition of the boundedness of the commutator on $\mathcal{M}_{q}^{p}(d x, w)$.

Theorem 1.21. Let $1<q \leq p<\infty, b \in \mathrm{BMO}$ and $T$ be a singular integral operator. Assume that $w \in H L M_{q}^{p}$ satisfies the weighted integral condition (2) for $p, q$ and $w$. Then we can extend the commutator $[b, T]$ as a bounded linear operator on $\mathcal{M}_{q}^{p}(d x, w)$ :

$$
\|[b, T] f\|_{\mathcal{M}_{q}^{p}(d x, w)} \leq C\|f\|_{\mathcal{M}_{q}^{p}(d x, w)} \quad\left(f \in \mathcal{M}_{q}^{p}(d x, w)\right) .
$$

This paper is organized as follows: In Section 2, we collect some important lemmas. In Section 3 we prove Theorems 1.3 and 1.6 related to the boundedness of the Hardy-Littlewood maximal operator. The key tool is the sparse family of cubes introduced in [13. Section 4 is devoted to the proof of Theorems 1.10 and 1.11 related to the sharp maximal inequalities. Here the decomposition by Lerner [26] plays an important role. In Section [5, we prove Theorems 1.17 and 1.21 and then give another application of the sharp maximal inequalities. In Section 6. we give the proof of some fundamental lemmas for the sake of completeness.

\section{Preliminaries}

We first recall an important property of the class $\mathcal{B}_{p, q}$.

Lemma 2.1 ([30]). Let $0<q \leq p<\infty$ and $w$ be a weight. Then $w \in \mathcal{B}_{p, q}$ if and only if $\left\|\chi_{Q_{0}}\right\|_{\mathcal{M}_{q}^{p}(d x, w)} \sim \Phi_{p, q, w}\left(Q_{0}\right)$ for any $Q_{0} \in \mathcal{Q}$.

We also prove an important relation combining the $\mathcal{B}_{p, q}$ condition and (7) as follows:

Lemma 2.2. Let $0<q \leq p<\infty$ and $w$ be a weight.

(1) $w$ satisfies (7) for all $Q \in \mathcal{Q}$, if and only if $w \in \mathcal{B}_{p, q}$ and

$$
\frac{1}{|Q|} \Phi_{p, q, w}(Q)\left\|w^{-\frac{1}{q}} \chi_{Q}\right\|_{H^{q^{\prime}, n(1-q / p)}} \leq C_{0}
$$

holds for all $Q \in \mathcal{Q}$. 
(2) For fixed $Q \in \mathcal{Q}, w$ satisfies (23), if and only if

$$
\frac{1}{|Q|} \int_{Q} f(x) d x \leq C_{0} \frac{\left\|f \cdot \chi_{Q}\right\|_{\mathcal{M}_{q}^{p}(d x, w)}}{\Phi_{p, q, w}(Q)}
$$

holds for any $f \geq 0$. Here, the constant $C_{0}$ in (24) is the same as the one of (23). Moreover, once $w$ satisfies (24) for all $Q$ with $C_{0}$ independent of $Q$, then $w$ is in the class $A_{q+1}$. In particular, $w$ is a doubling weight.

Proof. To show the assertion 1, we have only to prove $w \in \mathcal{B}_{p, q}$ by imposing (7) for all $Q \in \mathcal{Q}$. The converse is clear from the definition of $\Phi_{p, q, w}$. To this end, we recall the notion of the sparse family. Fix any $Q_{0} \in \mathcal{D}$ and let us show that

$$
\left\|\chi_{Q_{0}}\right\|_{\mathcal{M}_{q}^{p}(d x, w)} \sim_{n, p, q, w} \Phi_{p, q, w}\left(Q_{0}\right) .
$$

Set $\gamma_{0}:=\frac{w\left(Q_{0}\right)}{\left|Q_{0}\right|}$ and take $a \gg 2^{n}$. Define $\mathcal{D}_{0}:=\left\{Q_{0}\right\}$ and

$$
\mathcal{D}_{k}:=\left\{Q \in \mathcal{D}\left(Q_{0}\right): \frac{w(Q)}{|Q|}>a^{k} \gamma_{0}\right\} \quad(k \in \mathbb{N}) .
$$

By $\mathcal{D}_{k}^{*}:=\left\{Q_{j}^{k}\right\}_{j \in J_{k}}$, we denote the maximal subset of $\mathcal{D}_{k}$. The maximality implies that for each $Q_{j}^{k} \in \mathcal{D}_{k}^{*}$,

$$
a^{k} \gamma_{0}<\frac{w\left(Q_{j}^{k}\right)}{\left|Q_{j}^{k}\right|} \leq 2^{n} a^{k} \gamma_{0}
$$

Then we claim that the family $\left\{Q_{j}^{k}\right\}_{k \in \mathbb{N}_{0}, i \in J_{k}}$ is a sparse family. By the maximality, it is clear that $\left\{Q_{j}^{k}\right\}_{j \in J_{k}}$ is pairwise disjointed. Moreover, for any $Q_{j}^{k+1} \in \mathcal{D}_{k+1}^{*}$, it follows from the definition of $\mathcal{D}_{k+1}$ that $\frac{w\left(Q_{j}^{k+1}\right)}{\left|Q_{j}^{k+1}\right|}>a^{k} \gamma_{0}$ by $a>1$, which implies $Q_{j}^{k+1} \in \mathcal{D}_{k}$. Namely, we have $\Omega_{k+1} \subset \Omega_{k}$, where we denote

$$
\Omega_{k}:=\bigcup_{j \in J_{k}} Q_{j}^{k}
$$

We also have that

$$
\left|Q_{j}^{k} \cap \Omega_{k+1}\right| \leq \frac{2^{n}}{a}\left|Q_{j}^{k}\right|
$$

for any $k \in \mathbb{N}_{0}$ and $j \in J_{k}$. Indeed, since $\left\{Q_{i}^{k+1}\right\}_{i \in J_{k+1}}$ is disjoint, thanks to (26)

$$
\begin{aligned}
\left|Q_{j}^{k} \cap \Omega_{k+1}\right| & =\sum_{i \in J_{k+1}: Q_{j}^{k+1} \subset Q_{j}^{k}}\left|Q_{i}^{k+1}\right| \\
& \leq \sum_{i \in J_{k+1}: Q_{j}^{k+1} \subset Q_{j}^{k}} \frac{1}{a^{k+1} \gamma_{0}} w\left(Q_{i}^{k+1}\right) \\
& \leq \frac{1}{a^{k+1} \gamma_{0}} w\left(Q_{j}^{k}\right) \leq \frac{2^{n}}{a}\left|Q_{j}^{k}\right| .
\end{aligned}
$$

Now, it follows from (28) that $\frac{\left|Q_{j}^{k} \backslash \Omega_{k+1}\right|}{\left|Q_{j}^{k}\right|} \geq 2^{-1}$, for all $k \in \mathbb{N}_{0}$ and $j \in J_{k}$. In particular, we focus on the case of $k=0$ to obtain that $\frac{1}{Q_{0}} \int_{Q_{0}} \chi_{Q_{0} \backslash \Omega_{1}}(x) d x \geq 2^{-1}$. Using the dual inequality (5) and $a \gg 1$, we see that

$$
\frac{1}{2} \leq \frac{1}{\left|Q_{0}\right|}\left\|\chi_{Q_{0} \backslash \Omega_{1}}\right\|_{\mathcal{M}_{q}^{p}(d x, w)}\left\|w^{-\frac{1}{q}} \chi_{Q_{0}}\right\|_{H^{q^{\prime}, n(1-q / p)}} .
$$

Moreover, it follows from the assumption (7) that

$$
\left\|\chi_{Q_{0}}\right\|_{\mathcal{M}_{q}^{p}(d x, w)} \leq C\left\|\chi_{Q_{0} \backslash \Omega_{1}}\right\|_{\mathcal{M}_{q}^{p}(d x, w)} .
$$


Now, let us recall that

$$
\begin{aligned}
& \left\|\chi_{Q_{0} \backslash \Omega_{1}}\right\|_{\mathcal{M}_{q}^{p}(d x, w)}=\sup _{R \in \mathcal{D}\left(Q_{0}\right)}|R|^{\frac{1}{p}}\left(\frac{w\left(R \backslash \Omega_{1}\right)}{|R|}\right)^{\frac{1}{q}}, \\
& \Omega_{1}=\bigcup_{R \in \mathcal{D}_{1}} R, \quad \mathcal{D}_{1}=\left\{R \in \mathcal{D}\left(Q_{0}\right): \frac{w(R)}{|R|}>a \frac{w\left(Q_{0}\right)}{\left|Q_{0}\right|}\right\} .
\end{aligned}
$$

This implies that for any $R \in \mathcal{D}\left(Q_{0}\right) \backslash \mathcal{D}_{1}, w\left(R \backslash \Omega_{1}\right)=0$ and hence,

$$
\left\|\chi_{Q_{0} \backslash \Omega_{1}}\right\|_{\mathcal{M}_{q}^{p}(d x, w)}=\sup _{R \in \mathcal{D}\left(Q_{0}\right) \backslash \mathcal{D}_{1}}|R|^{\frac{1}{p}}\left(\frac{w\left(R \backslash \Omega_{1}\right)}{|R|}\right)^{\frac{1}{q}} \leq a^{\frac{1}{q}} \Phi_{p, q, w}\left(Q_{0}\right) .
$$

In summary, we obtain that

$$
\left\|\chi_{Q_{0}}\right\|_{\mathcal{M}_{q}^{p}(d x, w)} \leq C_{a, n, p, q, w} \Phi_{p, q, w}\left(Q_{0}\right) .
$$

Meanwhile, since the converse inequality is trivial, it follows $\left\|\chi_{Q_{0}}\right\|_{\mathcal{M}_{q}^{p}(d x, w)} \sim \Phi_{p, q, w}\left(Q_{0}\right)$ and hence $w \in \mathcal{B}_{p, q}$.

Next, let us show the assertion 2 Once we suppose (23), then we deduce (24) immediately from (5). Conversely, if we assume (24), then we employ (6) to obtain

$$
\begin{aligned}
\left\|w^{-\frac{1}{q}} \chi_{Q}\right\|_{H^{q^{\prime}, n(1-q / p)}} & =\sup _{f \geq 0:\left\|f \chi_{Q}\right\|_{\mathcal{M}_{q}^{p}(d x, w)} \leq 1} \int_{Q} f(x) d x \\
& \leq \sup _{f \geq 0:\left\|f \chi_{Q}\right\|_{\mathcal{M}_{q}^{p}(d x, w)} \leq 1} C_{0} \frac{|Q|}{\Phi_{p, q, w}(Q)}\left\|f \chi_{Q}\right\|_{\mathcal{M}_{q}^{p}(d x, w)} \\
& =C_{0} \frac{|Q|}{\Phi_{p, q, w}(Q)},
\end{aligned}
$$

which implies (23).

Finally, we shall show that $w$ is in $A_{q+1}$ by imposing (24) for all $Q \in \mathcal{Q}$. Note that

$$
\left\|w^{-\frac{1}{q}} \chi_{Q}\right\|_{\mathcal{M}_{q}^{p}(d x, w)}=\left\|\chi_{Q}\right\|_{\mathcal{M}_{q}^{p}(d x, d x)}=|Q|^{\frac{1}{p}} .
$$

With this in mind, by putting $f=w^{-\frac{1}{q}}$ in (24), then it follows that

$$
\frac{1}{|Q|} \int_{Q} w(x)^{-\frac{1}{q}} d x \leq C_{0} \frac{|Q|^{\frac{1}{p}}}{\Phi_{p, q, w}(Q)}=C_{0}\left(\frac{|Q|}{w(Q)}\right)^{\frac{1}{q}}
$$

which implies that $[w]_{A_{q+1}} \leq C_{0}$.

In [29], Nakai showed the self-improvement of the integral condition; see [32, Proposition 2.6] as well.

Proposition $2.3(29])$. Assume that a positive function $f:(0, \infty) \rightarrow(0, \infty)$ satisfies the integral condition:

$$
\int_{1}^{\infty} \frac{1}{f(r s)} \frac{d s}{s} \leq \frac{C_{0}}{f(r)} \quad(r>0)
$$

for some constant $C_{0}>0$. Then for all $\delta \in\left(0, C_{0}^{-1}\right)$, we have

$$
\int_{1}^{\infty} \frac{s^{\delta}}{f(r s)} \frac{d s}{s} \leq \frac{C_{0}}{1-C_{0} \delta} \frac{1}{f(r)} \quad(r>0)
$$

Similarly, we can show the self-improvement of the weighted integral condition. We will invoke the following observation in the proof of Theorem 1.21. 
Lemma 2.4. Let $0<q \leq p<\infty$ and $w$ satisfies the weighted integral condition (2) for $p, q$ and $w$. Then we have

$$
\int_{1}^{\infty} \frac{\log s}{\Phi_{p, q, w}(s Q)} \frac{d s}{s} \leq \frac{C}{\Phi_{p, q, w}(Q)} \quad(Q \in \mathcal{Q})
$$

or equivalently,

$$
\sum_{k=1}^{\infty} \frac{k}{\Phi_{p, q, w}\left(2^{k} Q\right)} \leq \frac{C}{\Phi_{p, q, w}(Q)} \quad(Q \in \mathcal{Q})
$$

Proof. Fix any cube $Q$. We put $f(s):=\Phi_{p, q, w}(s Q)$ for $s \geq 1$ and $f(s):=\Phi_{p, q, w}(Q)$ for $s \leq 1$. Since we assume the weighted integral condition (2) for $p, q$ and $w$, the integral condition for the above $f$ holds. Hence, we see that by Proposition 2.3.

$$
\int_{1}^{\infty} \frac{s^{\delta}}{\Phi_{p, q, w}(s Q)} \frac{d s}{s} \leq \frac{C}{\Phi_{p, q, w}(Q)}
$$

for some $\delta>0$. If we notice that $\log s \leq C_{\delta} s^{\delta}$, then we obtain (31). Note that the equivalence between (31) and (32) is clear.

Next, we collect the fundamental properties of the median and oscillation; see [13] for the details. Let $f \in L^{0}\left(\mathbb{R}^{n}\right), \lambda \in\left(0,2^{-1}\right)$ and $Q \in \mathcal{Q}$. For any median $m_{f}(Q)$, we have that

$$
\left|m_{f}(Q)\right| \leq\left(f \cdot \chi_{Q}\right)^{*}(\lambda|Q|), \quad \lim _{\ell(Q) \rightarrow 0, Q \ni x} m_{f}(Q)=f(x) \quad\left(\text { a.e. } x \in \mathbb{R}^{n}\right) .
$$

See [6, Lemma 2.2]. Here for the sake of readers' convenience, we supply a short proof in Section 6. Moreover, for the oscillation of $f$, we have that

$$
\omega_{\lambda}(f ; Q) \leq\left(\left(f-m_{f}(Q)\right) \chi_{Q}\right)^{*}(\lambda|Q|) \leq 2 \omega_{\lambda}(f ; Q) .
$$

Now, we introduce the notion of $w$-sparse family for $w \in A_{\infty}$. Let $w \in A_{\infty}$ and $\lambda_{w}^{\prime}<$ $2^{-1-2^{n+3}[w]_{A \infty}}$. We say that the family $\left\{Q_{j}^{k}\right\}_{k \in \mathbb{N}_{0}, j \in J_{k}}$ is a $w$-sparse family if the following properties hold:

(1) for each fixed $k \in \mathbb{N}_{0}$, the cubes $\left\{Q_{j}^{k}\right\}_{j \in J_{k}}$ are disjoint;

(2) if $\Omega_{k}:=\bigcup_{j \in J_{k}} Q_{j}^{k}$, then $\Omega_{k+1} \subset \Omega_{k}$;

(3) $\left|\Omega_{k+1} \cap Q_{j}^{k}\right| \leq \lambda_{w}^{\prime}\left|Q_{j}^{k}\right|$ for all $j \in J_{k}$.

The only difference from the original definition of the sparse family is the constant $\lambda_{w}^{\prime}$ appearing in the condition 3. That is, if we replace the constant $\lambda_{w}^{\prime}$ by $2^{-1}$ in the condition 3 , then the $w$-sparse family turns into the original sparse family introduced in [13]. The advantage of $w$-sparse family is as follows:

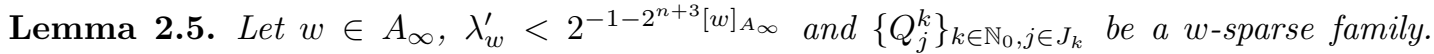
Then there exists a constant $C_{w}>1$ such that

$$
w\left(Q_{j}^{k}\right) \leq C_{w} w\left(Q_{j}^{k} \cap \Omega_{k+1}^{c}\right),
$$

holds for all $k \in \mathbb{N}_{0}$ and $j \in J_{k}$. Here, the constant $C_{w}$ can be written by

$$
C_{w}=\left(1-2\left(\lambda_{w}^{\prime}\right)^{\frac{\varepsilon}{1+\varepsilon}}\right)^{-1}, \quad \varepsilon:=\frac{1}{2^{n+3}[w]_{A_{\infty}}} .
$$


Proof. We can find such property in many papers; for example 9], but we give the complete proof. The proof is based on the reverse Hölder inequality. Since $\left\{Q_{j}^{k}\right\}_{k \in \mathbb{N}_{0}, j \in J_{k}}$ is a $w$-sparse family, we have that

$$
\begin{aligned}
w\left(Q_{j}^{k} \cap \Omega_{k+1}\right) & \leq\left(\int_{Q_{j}^{k}} w(x)^{1+\varepsilon} d x\right)^{\frac{1}{1+\varepsilon}} \cdot\left|\Omega_{k+1} \cap Q_{j}^{k}\right|^{\frac{\varepsilon}{1+\varepsilon}} \\
& \leq\left(\lambda_{w}^{\prime}\right)^{\frac{\varepsilon}{1+\varepsilon}}\left(\int_{Q_{j}^{k}} w(x)^{1+\varepsilon} d x\right)^{\frac{1}{1+\varepsilon}} \cdot\left|Q_{j}^{k}\right|^{\frac{\varepsilon}{1+\varepsilon}} .
\end{aligned}
$$

Hence, by the reverse Hölder inequality, we see that $w\left(Q_{j}^{k} \cap \Omega_{k+1}\right) \leq 2\left(\lambda_{w}^{\prime}\right)^{\frac{\varepsilon}{1+\varepsilon}} w\left(Q_{j}^{k}\right)$. Here,

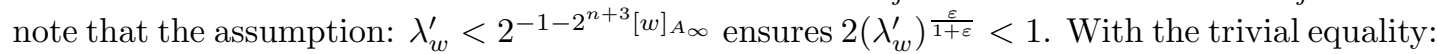
$w\left(Q_{j}^{k}\right)=w\left(Q_{j}^{k} \cap \Omega_{k+1}^{c}\right)+w\left(Q_{j}^{k} \cap \Omega_{k+1}\right)$ in mind, it follows that

$$
w\left(Q_{j}^{k}\right) \leq\left(1-2\left(\lambda_{w}^{\prime}\right)^{\frac{\varepsilon}{1+\varepsilon}}\right)^{-1} w\left(Q_{j}^{k} \cap \Omega_{k+1}^{c}\right) .
$$

We will invoke this observation to show Theorems 1.3 and 4.2 to follow. The latter one is a crucial part of Theorem 1.10 .

Here, we give an observation combining our results and the original sharp maximal inequalities. We will employ the following lemma to show Corollary 4.5.

Lemma 2.6. Let $f \in L^{0}\left(\mathbb{R}^{n}\right)$. Assume that $M f \in \mathcal{M}_{q_{0}}^{p_{0}}\left(v_{0}, w_{0}\right)$ for some $0<q_{0} \leq p_{0}<\infty$ and some weights $v_{0}, w_{0}$ satisfying that

$$
\lim _{l \rightarrow \infty} \Phi_{p_{0}, q_{0}, v_{0}, w_{0}}\left(2^{l} Q\right)=\infty, \quad(Q \in \mathcal{Q}), \quad \Phi_{p_{0}, q_{0}, v_{0}, w_{0}}(Q):=v_{0}(Q)^{\frac{1}{p_{0}}-\frac{1}{q_{0}}} w_{0}(Q)^{\frac{1}{q_{0}}} .
$$

Then for any $Q \in \mathcal{Q}$ and any medians $\left\{m_{f}\left(2^{l} Q\right)\right\}_{l \in \mathbb{N}_{0}}$, it holds that

$$
\lim _{l \rightarrow \infty} m_{f}\left(2^{l} Q\right)=0 .
$$

In particular, we have the following:

(1) If we assume that $M f \in \mathcal{M}_{q_{0}}^{p_{0}}\left(w_{0}, w_{0}\right)$ for some $0<q_{0} \leq p_{0}<\infty$ and $w_{0} \in A_{\infty}$. Then for any $Q \in \mathcal{Q}$ and any medians $\left\{m_{f}\left(2^{l} Q\right)\right\}_{l \in \mathbb{N}_{0}}$, (36) holds.

(2) If we assume that $M f \in \mathcal{M}_{q_{0}}^{p_{0}}\left(d x, w_{0}\right)$ for some $0<q_{0} \leq p_{0}<\infty$ and $w_{0} \in A_{\infty}$ satisfying the weighted integral condition (2) for $p_{0}, q_{0}$ and $w_{0}$. Then for any $Q \in \mathcal{Q}$ and any medians $\left\{m_{f}\left(2^{l} Q\right)\right\}_{l \in \mathbb{N}_{0}}$, (36) holds.

Proof. In view of (33), for $\lambda \in\left(0,2^{-1}\right)$, we have that

$$
\left|m_{f}\left(2^{l} Q\right)\right| \leq\left(f \cdot \chi_{2^{l} Q}\right)^{*}\left(\lambda\left|2^{l} Q\right|\right) \leq \frac{1}{\lambda\left|2^{l} Q\right|} \int_{0}^{\infty}\left(f \cdot \chi_{2^{l} Q}\right)^{*}(t) d t \leq \frac{1}{\lambda} \inf _{x \in 2^{l} Q} M f(x) .
$$

With the definition of the norm in mind, by taking $\lambda=4^{-1}$ for example, we notice that

$$
\left|m_{f}\left(2^{l} Q\right)\right| \lesssim \frac{1}{w_{0}\left(2^{l} Q\right)^{\frac{1}{q_{0}}}}\left(\int_{2^{l} Q} M f(x)^{q_{0}} d w_{0}(x)\right)^{\frac{1}{q_{0}}} \leq \frac{\|M f\|_{\mathcal{M}_{q_{0}}^{p_{0}}\left(v_{0}, w_{0}\right)}}{\Phi_{p_{0}, q_{0}, v_{0}, w_{0}}\left(2^{l} Q\right)} .
$$

By our assumption, $\|M f\|_{\mathcal{M}_{q_{0}}^{p_{0}}\left(v_{0}, w_{0}\right)}<\infty$. Hence, the assumption (35) yields the conclusion (36).

Particularly, let us consider the case of $v_{0}=w_{0} \in A_{\infty}$. The condition (35) reads $\lim _{l \rightarrow \infty} w_{0}\left(2^{l} Q\right)^{\frac{1}{p_{0}}}=$ $\infty$ for all $Q \in \mathcal{Q}$. Note that $w_{0} \in A_{\infty}$ implies $w_{0} \notin L^{1}(d x)$. In fact, by the reverse Hölder 
inequality, for any $R>0$, we have that

$$
\left(\frac{1}{|Q(R)|} \int_{Q(R)} w_{0}(x)^{1+\varepsilon} d x\right)^{\frac{1}{1+\varepsilon}} \leq \frac{2 w_{0}(Q(R))}{|Q(R)|},
$$

which implies

$$
|Q(R)|^{1-\frac{1}{1+\varepsilon}}\left(\int_{Q(R)} w_{0}(x)^{1+\varepsilon} d x\right)^{\frac{1}{1+\varepsilon}} \leq 2 w_{0}(Q(R)) .
$$

By tending $R \rightarrow \infty$, we obtain $\left\|w_{0}\right\|_{L^{1}(d x)}=\infty$. Thus, we see that $\lim _{l \rightarrow \infty} w_{0}\left(2^{l} Q\right)^{\frac{1}{p}}=$ $\left\|w_{0}\right\|_{L^{1}(d x)}^{\frac{1}{p}}=\infty$.

In the case of $v_{0}=d x$, the condition (35) reads $\lim _{l \rightarrow \infty} \Phi_{p_{0}, q_{0}, w_{0}}\left(2^{l} Q\right)=\infty$ for all $Q \in \mathcal{Q}$. However, this condition follows directly from the weighted integral condition (2) for $p_{0}, q_{0}$ and $w_{0}$.

Remark 2.7. The assumption (35) is essential in the following sense. If the assumption (35) does not hold, then $M f=f \equiv 1$ is in $\mathcal{M}_{q_{0}}^{p_{0}}\left(v_{0}, w_{0}\right)$, but (36) fails for any $Q \in \mathcal{Q}$.

To ensure the well-definedness of the singular integral operators and commutators on $\mathcal{M}_{q}^{p}(d x, w)$ in Theorems 1.17 and 1.21, we need the self-improvement property of the boundedness of $M$ on $\mathcal{M}_{q}^{p}(d x, w)$. The self-improvement property of $M$ on the Banach function spaces is obtained in [27, Theorem 1.2].

Lemma 2.8. Let $1<q \leq p<\infty$ and $w$ be a weight. Assume that the Hardy-Littlewood maximal operator $M$ is bounded on $\mathcal{M}_{q}^{p}(d x, w)$. Then there exists $r=r(p, q, w)>1$ such that $M^{(r)}$ is also bounded on $\mathcal{M}_{q}^{p}(d x, w)$.

Since the proof of Lemma2.8 is almost the same as the one of [27, Theorem 1.2], we postpone the proof to the Appendix. We can rephrase the assertion of Lemma 2.8 as follows: Once we obtain the boundedness of $M$ on $\mathcal{M}_{q}^{p}(d x, w)$ for some $1<q \leq p<\infty$ and some weight $w$, then there exists a small $\varepsilon \in(0,1)$ such that $M$ is also bounded on $\mathcal{M}_{q(1-\varepsilon)}^{p(1-\varepsilon)}(d x, w)$. This property yields the following corollary:

Corollary 2.9. Assume that the Hardy-Littlewood maximal operator $M$ is bounded on $\mathcal{M}_{q}^{p}(d x, w)$ for some $1<q \leq p<\infty$ and some weight $w$. Then for any $Q \in \mathcal{Q}$ and any $f \in \mathcal{M}_{q}^{p}(d x, w)$, $f \cdot \chi_{Q}$ is in $L^{\frac{1}{1-\varepsilon}}\left(\mathbb{R}^{n}\right)$.

Proof. Let $\varepsilon$ be as above. In this proof, we abbreviate $p(1-\varepsilon)$ and $q(1-\varepsilon)$ by $p_{\varepsilon}$ and $q_{\varepsilon}$ respectively. By the above observation, we know that $M$ is bounded on $\mathcal{M}_{q_{\varepsilon}}^{p_{\varepsilon}}(d x, w)$. By Theorem 1.5 and Lemma 2.2, it follows that

$$
\frac{1}{|Q|} \Phi_{p, q, w}(Q)^{\frac{1}{1-\varepsilon}}\left\|w^{-\frac{1}{q_{\varepsilon}}} \chi_{Q}\right\|_{H^{q_{\varepsilon}^{\prime}, n(1-q / p)}} \lesssim 1,
$$

for all $Q \in \mathcal{Q}$. Here, we used a simple fact that $\Phi_{p_{\varepsilon}, q_{\varepsilon}, w}(Q)=\Phi_{p, q, w}(Q)^{\frac{1}{1-\varepsilon}}$. With this in mind, by invoking the dual inequalisty (5), we have that

$$
\begin{aligned}
\int_{\mathbb{R}^{n}}\left|f(x) \chi_{Q}(x)\right|^{\frac{1}{1-\varepsilon}} d x & \leq C\left\||f|^{\frac{1}{1-\varepsilon}} w^{\frac{1}{q_{\varepsilon}}}\right\|_{\mathcal{M}_{q_{\varepsilon}}^{p_{\varepsilon}}(d x, d x)}\left\|w^{-\frac{1}{q_{\varepsilon}}} \chi_{Q}\right\|_{H^{q_{\varepsilon}^{\prime}, n(1-q / p)}} \\
& \leq C\left\||f|^{\frac{1}{1-\varepsilon}}\right\|_{\mathcal{M}_{q_{\varepsilon}}^{p_{\varepsilon}}(d x, w)} \frac{|Q|}{\Phi_{p, q, w}(Q)^{\frac{1}{1-\varepsilon}}} .
\end{aligned}
$$


If we notice that $\left\||f|^{\frac{1}{1-\varepsilon}}\right\|_{\mathcal{M}_{q \varepsilon}^{p_{\varepsilon}}(d x, w)}=\|f\|_{\mathcal{M}_{q}^{p}(d x, w)}^{\frac{1}{1-\varepsilon}}$, then we see that

$$
\left\|f \cdot \chi_{Q}\right\|_{L^{\frac{1}{1-\varepsilon}}\left(\mathbb{R}^{n}\right)} \leq C \frac{|Q|^{1-\varepsilon}}{\Phi_{p, q, w}(Q)}\|f\|_{\mathcal{M}_{q}^{p}(d x, w)}<\infty .
$$

To obtain the characterization of the boundedness of $M$ on $\mathcal{M}_{q}^{p}\left(d x, w_{\alpha}\right)$, we need to calculate the Choquet integral of the power function.

Lemma 2.10. Let $\beta \in(0,1-q / p)$. If the triple of $Q \in \mathcal{Q}$ contains 0 , then we have

$$
\int_{Q}|x|^{-n \beta} d H^{n(1-q / p)} \lesssim|Q|^{-\beta+(1-q / p)} .
$$

Proof. We let $R_{Q}:=4 \sqrt{n} \ell(Q)$. Since we know that $B\left(0, R_{Q}\right) \supset 3 Q$ by $0 \in 3 Q$, we have

$$
\begin{aligned}
\int_{Q}|x|^{-n \beta} d H^{n(1-q / p)} \leq & \int_{0}^{\infty} H^{n(1-q / p)}\left(\left\{x \in B\left(0, R_{Q}\right):|x|^{-n \beta}>t\right\}\right) d t \\
= & \int_{0}^{R_{Q}^{-n \beta}} H^{n(1-q / p)}\left(\left\{x \in B\left(0, R_{Q}\right):|x|^{-n \beta}>t\right\}\right) d t \\
& +\int_{R_{Q}^{-n \beta}}^{\infty} H^{n(1-q / p)}\left(\left\{x \in B\left(0, R_{Q}\right):|x|^{-n \beta}>t\right\}\right) d t \\
= & R_{Q}^{-n \beta} H^{n(1-q / p)}\left(B\left(0, R_{Q}\right)\right)+\int_{R_{Q}^{-n \beta}}^{\infty} H^{n(1-q / p)}\left(B\left(0, t^{-\frac{1}{n \beta}}\right)\right) d t \\
\sim & |Q|^{-\beta+(1-q / p)}+\int_{R_{Q}^{-n \beta}}^{\infty} t^{-\frac{1-q / p}{\beta}} d t .
\end{aligned}
$$

Since $\beta<1-q / p, g(t):=t^{-\frac{1-q / p}{\beta}}$ is integrable on $\left[R_{Q}^{-n \beta}, \infty\right)$, and hence, we obtain that

$$
\int_{Q}|x|^{-n \beta} d H^{n(1-q / p)} \lesssim|Q|^{-\beta+(1-q / p)} .
$$

We will invoke Lemma 2.10 in the proof of Lemma 3.1

\section{Proof of Theorems 1.3 and 1.6}

We first show Theorem 1.3 .

Proof of Theorem 1.3. Assume the boundedness of $M$ on $\mathcal{M}_{q}^{p}(d x, w)$. First, we shall prove $w \in \mathcal{B}_{p, q}$. The crucial part is proved in Lemma 2.2, In fact, once we suppose the boundedness of $M$ on $\mathcal{M}_{q}^{p}(d x, w)$, then it follows (7) by Theorem 1.5. Then we invoke Lemma 2.2 to obtain $w \in \mathcal{B}_{p, q}$.

Next, let us show $w \in A_{q+1}$. To this end, we write $\sigma^{*}:=w^{-\frac{1}{q}}$. We fix any $Q_{0} \in \mathcal{D}$ and calculate the norm of $\chi_{Q_{0}} \cdot \sigma^{*}$.

$$
\left\|\chi_{Q_{0}} \cdot \sigma^{*}\right\|_{\mathcal{M}_{q}^{p}(d x, w)}=\left\|\chi_{Q_{0}}\right\|_{\mathcal{M}_{q}^{p}(d x, d x)}=\left|Q_{0}\right|^{\frac{1}{p}} .
$$


Since, we have the pointwise estimate: $\chi_{Q_{0}}(x) \frac{\sigma^{*}\left(Q_{0}\right)}{\left|Q_{0}\right|} \leq M\left[\chi_{Q_{0}} \cdot \sigma^{*}\right](x)$, the boundedness of $M$ implies that

$$
\frac{\sigma^{*}\left(Q_{0}\right)}{\left|Q_{0}\right|}\left\|\chi_{Q_{0}}\right\|_{\mathcal{M}_{q}^{p}(d x, w)} \leq\|M\|_{\mathcal{M}_{q}^{p}(d x, w) \rightarrow \mathcal{M}_{q}^{p}(d x, w)}\left\|\chi_{Q_{0}} \sigma^{*}\right\|_{\mathcal{M}_{q}^{p}(d x, w)} \sim\left|Q_{0}\right|^{\frac{1}{p}} .
$$

If we notice that $\left\|\chi_{Q_{0}}\right\|_{\mathcal{M}_{q}^{p}(d x, w)} \geq \Phi_{p, q, w}\left(Q_{0}\right)$, then it follows that

$$
\left(\frac{w\left(Q_{0}\right)}{\left|Q_{0}\right|}\right)^{\frac{1}{q}} \frac{\sigma^{*}\left(Q_{0}\right)}{\left|Q_{0}\right|} \leq\|M\|_{\mathcal{M}_{q}^{p}(d x, w) \rightarrow \mathcal{M}_{q}^{p}(d x, w)},
$$

which implies $w \in A_{q+1}$ with $[w]_{A_{q+1}} \leq\|M\|_{\mathcal{M}_{q}^{p}(d x, w) \rightarrow \mathcal{M}_{q}^{p}(d x, w)}^{\frac{1}{q}}$.

To prove (b), we assume $w^{-\frac{1}{q-1}} \in \mathcal{B}_{p, q}$ additionally. Let us show $w \in A_{q}$. To this end, we fix any $Q \in \mathcal{D}$ and write $\sigma:=w^{-\frac{1}{q-1}}$. Since $\sigma \in \mathcal{B}_{p, q}$ is a dual weight of $w: \sigma^{q} w=\sigma$, we notice that

$$
\left\|\chi_{Q} \cdot \sigma\right\|_{\mathcal{M}_{q}^{p}(d x, w)}=\left\|\chi_{Q}\right\|_{\mathcal{M}_{q}^{p}(d x, \sigma)} \sim|Q|^{\frac{1}{p}}\left(\frac{\sigma(Q)}{|Q|}\right)^{\frac{1}{q}} .
$$

On the other hand, if we notice that $\frac{\sigma(Q)}{|Q|} \chi_{Q}(x) \leq M\left[\chi_{Q} \cdot \sigma\right](x)$, then the boundedness of $M$ on $\mathcal{M}_{q}^{p}(d x, w)$ yields that

$$
\frac{\sigma(Q)}{|Q|}\left\|\chi_{Q}\right\|_{\mathcal{M}_{q}^{p}(d x, w)} \leq\left\|M\left[\chi_{Q} \cdot \sigma\right]\right\|_{\mathcal{M}_{q}^{p}(d x, w)} \lesssim\left\|\chi_{Q} \cdot \sigma\right\|_{\mathcal{M}_{q}^{p}(d x, w)} \sim|Q|^{\frac{1}{p}}\left(\frac{\sigma(Q)}{|Q|}\right)^{\frac{1}{q}} .
$$

Moreover, since we know that $\left\|\chi_{Q}\right\|_{\mathcal{M}_{q}^{p}(d x, w)} \geq|Q|^{\frac{1}{p}}\left(\frac{w(Q)}{|Q|}\right)^{\frac{1}{q}}$, by dividing the both terms by $|Q|^{\frac{1}{p}}$, it follows that

$$
\frac{\sigma(Q)}{|Q|}\left(\frac{w(Q)}{|Q|}\right)^{\frac{1}{q}} \leq C_{0}\left(\frac{\sigma(Q)}{|Q|}\right)^{\frac{1}{q}}
$$

or equivalently,

$$
\frac{w(Q)}{|Q|}\left(\frac{1}{|Q|} \int_{Q} w(x)^{-\frac{1}{q-1}} d x\right)^{q-1} \leq C_{0}^{q}
$$

which implies $w \in A_{q}$.

Finally, to prove (c), we assume $\sigma:=w^{-\frac{1}{q-1}} \in A_{\infty}$. Let us show $w \in A_{q}$. Since we already know that $w, w^{-\frac{1}{q-1}} \in A_{\infty}$ implies $w \in A_{q}$; see [9], the assertion (c) is immediately. However, we give another proof depending on the structure of Morrey spaces here. Fix any $Q_{0} \in \mathcal{D}$ and by the Calderón-Zygmund decomposition, we shall construct a $\sigma$-sparse family $\left\{Q_{j}^{k}\right\}_{k \in \mathbb{N}_{0}, j \in J_{k}}$ as in (25). Now, we set $\gamma_{0}:=\frac{1}{\left|Q_{0}\right|} \int_{Q_{0}} \sigma(y) d y$ and take a large $a_{[\sigma]_{A_{\infty}}}>2^{n}$ so that $2^{n} / a_{[\sigma]_{A_{\infty}}} \leq \lambda_{\sigma}^{\prime}$. Then we define $\mathcal{D}_{0}:=\left\{Q_{0}\right\}$ and

$$
\mathcal{D}_{k}:=\left\{Q \in \mathcal{D}\left(Q_{0}\right): \frac{\sigma(Q)}{|Q|}>a_{[\sigma]_{A_{\infty}}}^{k} \gamma_{0}\right\} \quad(k \in \mathbb{N}) .
$$

We denote the maximal subset of $\mathcal{D}_{k}$ by $\mathcal{D}_{k}^{*}:=\left\{Q_{j}^{k}\right\}_{j \in J_{k}}$ again. In view of (28) and $2^{n} / a_{[\sigma]_{A_{\infty}}} \leq$ $\lambda_{\sigma}^{\prime}$, we see that $\left\{Q_{j}^{k}\right\}_{k \in \mathbb{N}_{0}, j \in J_{k}}$ is a $\sigma$-sparse family. In particular, by Lemma 2.5, it follows that

$$
\sigma\left(Q_{0} \backslash \Omega_{1}\right) \geq C_{[\sigma]_{A_{\infty}}} \sigma\left(Q_{0}\right),
$$

where $\Omega_{1}:=\bigcup_{j \in J_{1}} Q_{j}^{1}=\bigcup_{R \in \mathcal{D}_{1}} R$. This implies that

$$
\chi_{Q_{0}}(x) \frac{\sigma\left(Q_{0}\right)}{\left|Q_{0}\right|} \lesssim[\sigma]_{A_{\infty}} \chi_{Q_{0}}(x) \frac{\sigma\left(Q_{0} \backslash \Omega_{1}\right)}{\left|Q_{0}\right|} \leq M\left[\chi_{Q_{0} \backslash \Omega_{1}} \cdot \sigma\right](x) .
$$


By taking the weighted Morrey norm of the both side and using the boundedness of $M$ and $\sigma^{q} \cdot w=\sigma$, it follows that

$$
\frac{\sigma\left(Q_{0}\right)}{\left|Q_{0}\right|}\left\|\chi_{Q_{0}}\right\|_{\mathcal{M}_{q}^{p}(d x, w)} \lesssim_{[\sigma]_{A_{\infty}}}\left\|\chi_{Q_{0} \backslash \Omega_{1}} \cdot \sigma\right\|_{\mathcal{M}_{q}^{p}(d x, w)}=\left\|\chi_{Q_{0} \backslash \Omega_{1}}\right\|_{\mathcal{M}_{q}^{p}(d x, \sigma)}
$$

By recalling that

$$
\Omega_{1}=\bigcup_{R \in \mathcal{D}_{1}} R, \quad \mathcal{D}_{1}:=\left\{R \in \mathcal{D}\left(Q_{0}\right): \frac{\sigma(R)}{|R|}>a_{[\sigma]_{A_{\infty}}} \frac{\sigma\left(Q_{0}\right)}{\left|Q_{0}\right|}\right\},
$$

we see that $\left\|\chi_{Q_{0} \backslash \Omega_{1}}\right\|_{\mathcal{M}_{q}^{p}(d x, \sigma)} \leq a_{[\sigma]]_{\infty}}^{\frac{1}{q}}\left|Q_{0}\right|^{\frac{1}{p}}\left(\frac{\sigma\left(Q_{0}\right)}{\left|Q_{0}\right|}\right)^{\frac{1}{q}}$. Meanwhile, we know that $\left\|\chi_{Q_{0}}\right\|_{\mathcal{M}_{q}^{p}(d x, w)} \geq$ $\left|Q_{0}\right|^{\frac{1}{p}}\left(\frac{w\left(Q_{0}\right)}{\left|Q_{0}\right|}\right)^{\frac{1}{q}}$. As a result, by inserting these two estimates to (39), we obtain that

$$
\frac{\sigma\left(Q_{0}\right)}{\left|Q_{0}\right|}\left(\frac{w\left(Q_{0}\right)}{\left|Q_{0}\right|}\right)^{\frac{1}{q}} \lesssim_{[\sigma]_{A_{\infty}}}\left(\frac{\sigma\left(Q_{0}\right)}{\left|Q_{0}\right|}\right)^{\frac{1}{q}}
$$

which implies $w \in A_{q}$.

Next, we shall prove Theorem 1.6 and reprove the characterization of the weak type boundedness of $M$ on $\mathcal{M}_{q}^{p}\left(d x, w_{\alpha}\right)$ with $w_{\alpha}(x)=|x|^{\alpha}$.

Proof of Theorem 1.6. Let us fix any non-negative $f \in \mathcal{M}_{q}^{p}(d x, w), Q_{0} \in \mathcal{D}$ and $t>0$. It suffices to show the inequality

$$
\left|Q_{0}\right|^{\frac{1}{p}-\frac{1}{q}} t w\left(\left\{x \in Q_{0}: M f(x)>t\right\}\right)^{\frac{1}{q}} \lesssim_{p, q, w}\|f\|_{\mathcal{M}_{q}^{p}(d x, w)} .
$$

We define

$$
\mathcal{D}_{t}:=\left\{Q \in \mathcal{D}\left(Q_{0}\right): \frac{1}{|Q|} \int_{Q} f(x) d x>t\right\},
$$

and denote the maximal cubes of $\mathcal{D}_{t}$ by $\mathcal{D}_{t}^{*}$. By the disjointness of $\mathcal{D}_{t}^{*}$, it is easy to see that $w\left(\left\{x \in Q_{0}: M f(x)>t\right\}\right)=\sum_{Q \in \mathcal{D}_{t}^{*}} w(Q)$. Hence, it follows that

$$
w\left(\left\{x \in Q_{0}: M f(x)>t\right\}\right)<\sum_{Q \in \mathcal{D}_{t}^{*}} \frac{w(Q)}{|Q|^{q}} t^{-q}\left(\int_{Q} f(x) d x\right)^{q} .
$$

By our assumption, we take $b_{Q_{0}} \in \mathfrak{B}_{n(1-q / p)}$ satsfying (12). Using Hölder's inequality, we have that

$$
\begin{aligned}
& w\left(\left\{x \in Q_{0}: M f(x)>t\right\}\right) \\
< & \sum_{Q \in \mathcal{D}_{t}^{*}} \frac{w(Q)}{|Q|} t^{-q}\left\|f \chi_{Q}\right\|_{L^{q}\left(b_{Q_{0}} w\right)}^{q}\left(\frac{1}{|Q|} \int_{Q}\left[b_{Q_{0}}(x) w(x)\right]^{-\frac{q^{\prime}}{q}} d x\right)^{\frac{q}{q^{\prime}}} \\
\lesssim & t^{-q}\left|Q_{0}\right|^{1-\frac{q}{p}}\|f\|_{L^{q}\left(b_{Q_{0}} w\right)}^{q} \lesssim t^{-q}\left|Q_{0}\right|^{1-\frac{q}{p}}\|f\|_{\mathcal{M}_{q}^{p}(d x, w)},
\end{aligned}
$$

which implies (40).

The proof of the following lemma is based on [41, Proposition 4.2].

Lemma 3.1. Let $1<q \leq p<\infty$ and $w_{\alpha}(x):=|x|^{\alpha}$. If we assume that $-\frac{q}{p} n \leq \alpha<n\left(q-\frac{q}{p}\right)$, then for any $Q_{0} \in \mathcal{Q}$, there exists $b_{Q_{0}} \in \mathfrak{B}_{n(1-q / p)}$ satisfying (12) for any $Q \in \mathcal{D}\left(Q_{0}\right)$. 
Proof. Fix any $Q_{0} \in \mathcal{D}$ and decompose the proof into the two cases.

(Case.1: $0 \notin 3 Q_{0}$ ) In this case, by the geometrical observation, we see that for any $Q \in$ $\mathcal{D}\left(Q_{0}\right), 0 \notin 3 Q$ holds. We define

$$
b_{Q_{0}}:=\frac{\left(M \chi_{Q_{0}}\right)^{(1-q / p)+\varepsilon}}{\left|Q_{0}\right|^{1-q / p}},
$$

where $\varepsilon \in(0, q / p)$ is a fixed small number. Note that $\int_{Q_{0}} b_{Q_{0}} d H^{n(1-q / p)} \lesssim_{p, q} 1$; see 34, Lemma. 1]. Let us check (12). We notice that $b_{Q_{0}}(x)=\left|Q_{0}\right|^{q / p-1}$ for $x \in Q_{0}$ and that $w_{\beta}(Q) /|Q| \sim|c(Q)|^{\beta}$ for any $\beta \in \mathbb{R}$ and any $Q \in \mathcal{D}\left(Q_{0}\right)$. Hence, we have that

$$
\int_{Q}\left(b_{Q_{0}}(x) w_{\alpha}(x)\right)^{-\frac{q^{\prime}}{q}} d x=w_{-\alpha \frac{q^{\prime}}{q}}(Q) \cdot\left|Q_{0}\right|^{(1-q / p) \frac{q^{\prime}}{q}} \sim \frac{|c(Q)|^{-\alpha \frac{q^{\prime}}{q}}}{|Q|^{-1}}\left|Q_{0}\right|^{(1-q / p) \frac{q^{\prime}}{q}} .
$$

Meanwhile, since we have $w_{\alpha}(Q) /|Q| \sim|c(Q)|^{\alpha}$, it follows that

$$
\begin{aligned}
& \frac{1}{|Q|} \Phi_{p, q, w_{\alpha}}(Q)^{q}\left(\frac{1}{|Q|} \int_{Q}\left(b_{Q_{0}}(x) w_{\alpha}(x)\right)^{-\frac{q^{\prime}}{q}} d x\right)^{\frac{q}{q^{\prime}}} \\
\sim & |Q|^{\frac{q}{p}-1}|c(Q)|^{\alpha}\left(|c(Q)|^{-\alpha \frac{q^{\prime}}{q}}\left|Q_{0}\right|^{(1-q / p) \frac{q^{\prime}}{q}}\right)^{\frac{q}{q^{\prime}}}=\left(\frac{\left|Q_{0}\right|}{|Q|}\right)^{1-q / p} .
\end{aligned}
$$

Therefore, we see that $b_{Q_{0}}$ satisfies (12).

(Case. 2: $0 \in 3 Q_{0}$ ) In this case, we define

$$
b_{Q_{0}}(x):=\left|Q_{0}\right|^{\beta-(1-q / p)}|x|^{-n \beta},
$$

where we take $\beta>0$ so that

$$
\alpha<n \beta+n(q-1)<n(1-q / p)+n(q-1) .
$$

First, we check $\int_{Q_{0}} b_{Q_{0}} d H^{n(1-q / p)} \lesssim_{p, q} 1$. Since $3 Q_{0} \ni 0$, by Lemma 2.10, it follows that

$$
\int_{Q_{0}}|x|^{-n \beta} d H^{n(1-q / p)} \lesssim\left|Q_{0}\right|^{-\beta+(1-q / p)} .
$$

Thus, we see that

$$
\int_{Q_{0}} b_{Q_{0}} d H^{n(1-q / p)}=\left|Q_{0}\right|^{\beta-(1-q / p)} \int_{Q_{0}}|x|^{-n \beta} d H^{n(1-q / p)} \lesssim 1 .
$$

Next, we check the complicated part of (12):

$$
\frac{1}{|Q|} \Phi_{p, q, w_{\alpha}}(Q)^{q}\left(\frac{1}{|Q|} \int_{Q}\left[b_{Q_{0}}(x) w_{\alpha}(x)\right]^{-\frac{q^{\prime}}{q}} d x\right)^{\frac{q}{q^{\prime}}} \lesssim\left(\frac{\left|Q_{0}\right|}{|Q|}\right)^{1-q / p} .
$$

Since we proved the case of $0 \notin 3 Q$ in (Case. 1), we have only to show that in the case of $0 \in 3 Q$. The left-hand side of (42) equals to

$$
\begin{aligned}
& \frac{w_{\alpha}(Q)}{|Q|}\left(\frac{1}{|Q|} \int_{Q}\left[w_{\alpha-n \beta}(x)\right]^{-\frac{q^{\prime}}{q}} d x\right)^{\frac{q}{q^{\prime}}} \frac{\left|Q_{0}\right|^{1-q / p-\beta}}{|Q|^{1-q / p}} \\
\sim & \frac{w_{\alpha-n \beta}(Q)}{|Q|}\left(\frac{1}{|Q|} \int_{Q}\left[w_{\alpha-n \beta}(x)\right]^{-\frac{q^{\prime}}{q}} d x\right)^{\frac{q}{q^{\prime}}} \frac{\left|Q_{0}\right|^{1-q / p-\beta}}{|Q|^{1-q / p-\beta}} .
\end{aligned}
$$

Note that $w_{\alpha-n \beta} \in A_{q}$ by $\alpha-n \beta<n(q-1)$ and that $\left(|Q| /\left|Q_{0}\right|\right)^{\beta} \leq 1$. Hence, we have (42) and constructed the desired functions $\left\{b_{Q_{0}}\right\}_{Q_{0} \in \mathcal{D}}$. 


\section{Proof of Theorems 1.10 And 1.11}

Our first observation is to generalize Theorem 1.9 to the $w$-sparse family setting.

Proposition 4.1. Let $w \in A_{\infty}, \lambda_{w}^{\prime}<2^{-1-2^{n+3}[w]_{A_{\infty}}}$ and $\lambda_{w}:=2^{-n-2} \lambda_{w}^{\prime}$. For $Q_{0} \in \mathcal{Q}$ and $f: Q_{0} \rightarrow \mathbb{R}$, there exists a w-sparse family $\left\{Q_{j}^{k}\right\}_{k \in \mathbb{N}_{0}, j \in J_{k}} \subset \mathcal{D}\left(Q_{0}\right)$ such that for a.e. $x \in \mathbb{R}^{n}$,

$$
\left|f(x)-m_{f}\left(Q_{0}\right)\right| \leq 4 M_{\lambda_{w} ; Q_{0}}^{\sharp, d} f(x)+2 \sum_{k \in \mathbb{N}_{0}} \sum_{j \in J_{k}} \omega_{\lambda_{w}}\left(f ; Q_{j}^{k}\right) \chi_{Q_{j}^{k}}(x) .
$$

Since we need only a slight modification of the proof of Theorem 1.9 to show Proposition 4.1. we omit the proof here; see Appendix for the detail. By employing Proposition 4.1, we can show the following local estimate.

Theorem 4.2. Let $0<q, s<\infty, w, v \in A_{\infty}$ and $Q_{0} \in \mathcal{Q}$. We choose $\lambda_{w}^{\prime}>0$ so that

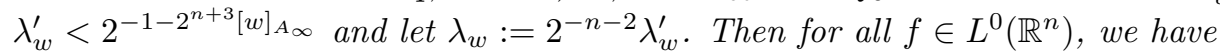

$$
\begin{aligned}
\left(\int_{Q_{0}}|f(x)|^{q} d w(x)\right)^{\frac{1}{q}} \lesssim_{q, s, w, v} & \left(\int_{Q_{0}} M_{\lambda_{w} ; Q_{0}}^{\sharp, d} f(x)^{q} d w(x)\right)^{\frac{1}{q}} \\
& +w\left(Q_{0}\right)^{\frac{1}{q}}\left(\frac{1}{v\left(Q_{0}\right)} \int_{Q_{0}}|f(x)|^{s} d v(x)\right)^{\frac{1}{s}} .
\end{aligned}
$$

Proof. We take a median $m_{f}\left(Q_{0}\right)$ and use the quasi-triangle inequality to get

$$
\left(\int_{Q_{0}}|f(x)|^{q} d w(x)\right)^{\frac{1}{q}} \lesssim q\left(\int_{Q_{0}}\left|f(x)-m_{f}\left(Q_{0}\right)\right|^{q} d w(x)\right)^{\frac{1}{q}}+w\left(Q_{0}\right)^{\frac{1}{q}}\left|m_{f}\left(Q_{0}\right)\right|
$$

For the first term, by applying Proposition 4.1, we obtain that

$$
\begin{gathered}
\left(\int_{Q_{0}}\left|f(x)-m_{f}\left(Q_{0}\right)\right|^{q} d w(x)\right)^{\frac{1}{q}} \\
\lesssim_{q}\left(\int_{Q_{0}} M_{\lambda_{w} ; Q_{0}}^{\sharp, d} f(x)^{q} d w(x)\right)^{\frac{1}{q}}+\left[\int_{Q_{0}}\left(\sum_{k \in \mathbb{N}_{0}} \sum_{j \in J_{k}} \omega_{\lambda_{w}}\left(f ; Q_{j}^{k}\right) \chi_{Q_{j}^{k}}(x)\right)^{q} d w(x)\right]^{\frac{1}{q}} .
\end{gathered}
$$

Now, we focus on

$$
\mathrm{I}:=\left[\int_{Q_{0}}\left(\sum_{k \in \mathbb{N}_{0}} \sum_{j \in J_{k}} \omega_{\lambda_{w}}\left(f ; Q_{j}^{k}\right) \chi_{Q_{j}^{k}}(x)\right)^{q} d w(x)\right]^{\frac{1}{q}}
$$

We recall that $\left\{Q_{j}^{k}\right\}_{k \in \mathbb{N}_{0}, j \in J_{k}}$ is a $w$-sparse family, in particular, $w\left(Q_{j}^{k}\right) \leq C_{w} w\left(Q_{j}^{k} \cap \Omega_{k+1}^{c}\right)$ holds by Lemma 2.5, where $\Omega_{k+1}$ is given by (27). This implies that $\chi_{Q_{j}^{k}}(x) \leq C_{w} M_{w}\left[\chi_{Q_{j}^{k} \cap \Omega_{k+1}^{c}}\right](x)$, where the weighted Hardy-Littlewood maximal operator $M_{w}$ is defined by

$$
M_{w} f(x):=\sup _{Q \in \mathcal{Q}} \frac{\chi_{Q}(x)}{w(Q)} \int_{Q}|f(y)| d w(y)
$$


Hence, by taking $\eta>\max \left(1, q^{-1}\right)$ and employing the boundedness of $M_{w}$ on $L^{\eta q}\left(\ell^{\eta}\right)(w)$, it follows that

$$
\begin{aligned}
\mathrm{I} & \lesssim w w \\
\lesssim & {\left[\int_{Q_{0}}\left(\sum_{k \in \mathbb{N}_{0}} \sum_{j \in J_{k}} \omega_{\lambda_{w}}\left(f ; Q_{j}^{k}\right) M_{w}\left[\chi_{Q_{j}^{k} \cap \Omega_{k+1}^{c}}\right](x)^{\eta}\right)^{q} d w(x)\right]^{\frac{1}{q}} } \\
\lesssim q, w & {\left[\int_{\mathbb{R}^{n}}\left(\sum_{k \in \mathbb{N}_{0}} \sum_{j \in J_{k}} \omega_{\lambda_{w}}\left(f ; Q_{j}^{k}\right) \chi_{Q_{j}^{k} \cap \Omega_{k+1}^{c}}(x)\right)^{q} d w(x)\right]^{\frac{1}{q}} . }
\end{aligned}
$$

If we notice that $\omega_{\lambda_{w}}\left(f ; Q_{j}^{k}\right) \chi_{Q_{j}^{k}}(x) \leq M_{\lambda_{w} ; Q_{0}}^{\sharp, d} f(x)$ and that $\sum_{k \in \mathbb{N}_{0}} \sum_{j \in J_{k}} \chi_{Q^{k_{j}} \cap \Omega_{k+1}^{c}} \leq \chi_{Q_{0}}$ by the disjointness of $\left\{Q_{j}^{k} \cap \Omega_{k+1}^{c}\right\}_{k \in \mathbb{N}_{0}, j \in J_{k}}$, then we see that

$$
\mathrm{I} \lesssim_{q, w}\left(\int_{Q_{0}} M_{\lambda_{w} ; Q_{0}}^{\sharp, d} f(x)^{q} d w(x)\right)^{\frac{1}{q}} .
$$

Next, we evaluate the second term: $w\left(Q_{0}\right)^{\frac{1}{q}}\left|m_{f}\left(Q_{0}\right)\right|$. Since $v \in A_{\infty}$, we can find $u \in(1, \infty)$ such that $v \in A_{u}$. We set $r:=\frac{s}{u}$. By (33), for $\lambda \in\left(0,2^{-1}\right)$, we have that

$$
\begin{aligned}
\left|m_{f}\left(Q_{0}\right)\right| & \leq\left(f \cdot \chi_{Q_{0}}\right)^{*}\left(\lambda\left|Q_{0}\right|\right) \\
& \leq\left(\frac{1}{\lambda\left|Q_{0}\right|} \int_{0}^{\lambda\left|Q_{0}\right|}\left(f \cdot \chi_{Q_{0}}\right)^{*}(t)^{r} d t\right)^{\frac{1}{r}} \\
& \lesssim_{r}\left(\frac{1}{\left|Q_{0}\right|} \int_{Q_{0}}|f(x)|^{r} d x\right)^{\frac{1}{r}} .
\end{aligned}
$$

Moreover, by $v \in A_{u}$ and $r u=s$, we see that

$$
\begin{aligned}
\left|m_{f}\left(Q_{0}\right)\right| & \lesssim_{r}[v]_{A_{u}}^{\frac{1}{r u}}\left(\frac{1}{\left|Q_{0}\right|} \int_{Q_{0}}|f(x)|^{r u} d v(x)\right)^{\frac{1}{r u}} \cdot\left(\frac{\left|Q_{0}\right|}{v\left(Q_{0}\right)}\right)^{\frac{1}{r u}} \\
& =[v]_{A_{u}}^{\frac{1}{s}}\left(\frac{1}{v\left(Q_{0}\right)} \int_{Q_{0}}|f(x)|^{s} d v(x)\right)^{\frac{1}{s}} .
\end{aligned}
$$

Thus, we complete the proof.

As a corollary, we obtain the following:

Corollary 4.3. Let $0<q \leq p<\infty, s>0$, $w_{1}$ be a weight, and $w_{2}, v \in A_{\infty}$. Then for any $f \in L^{0}\left(\mathbb{R}^{n}\right)$, one has

$$
\begin{aligned}
\|f\|_{\mathcal{M}_{q}^{p}\left(w_{1}, w_{2}\right)} \lesssim_{q, s, w_{2}, v} & \left\|M_{\lambda_{w}}^{\sharp, d} f\right\|_{\mathcal{M}_{q}^{p}\left(w_{1}, w_{2}\right)} \\
& +\sup _{Q \in \mathcal{Q}} w_{1}(Q)^{\frac{1}{p}}\left(\frac{w_{2}(Q)}{w_{1}(Q)}\right)^{\frac{1}{q}}\left(\frac{1}{v(Q)} \int_{Q}|f(x)|^{s} v(x) d x\right)^{\frac{1}{s}} .
\end{aligned}
$$

In particular, the following two types of weighted sharp maximal inequalities hold:

(1) (Komori-Shirai type: $w_{1}=w_{2}=v=w \in A_{\infty}$ )

$$
\|f\|_{\mathcal{M}_{q}^{p}(w, w)} \lesssim s, q, w\left\|M_{\lambda_{w}}^{\sharp, d} f\right\|_{\mathcal{M}_{q}^{p}(w, w)}+\|f\|_{\mathcal{M}_{s}^{p}(w, w)} .
$$


(2) (Samko type: $w_{1}=d x, w_{2}=v=w \in A_{\infty}$ )

$$
\begin{aligned}
& \|f\|_{\mathcal{M}_{q}^{p}(d x, w)} \\
& \quad \lesssim_{s, q, w}\left\|M_{\lambda_{w}}^{\sharp, d} f\right\|_{\mathcal{M}_{q}^{p}(d x, w)}+\sup _{Q \in \mathcal{Q}} \Phi_{p, q, w}(Q)\left(\frac{1}{w(Q)} \int_{Q}|f(x)|^{s} d w(x)\right)^{\frac{1}{s}} .
\end{aligned}
$$

Next, we consider the converse inequality.

Proposition 4.4. Let $0<q \leq p<\infty, \lambda \in\left(0,2^{-1}\right)$ and $w \in A_{\infty}$. Then for $f \in L^{0}\left(\mathbb{R}^{n}\right)$, we have that

$$
\left\|M_{\lambda}^{\sharp, d} f\right\|_{\mathcal{M}_{q}^{p}(w, w)} \lesssim_{\lambda, p, q, w}\|f\|_{\mathcal{M}_{q}^{p}(w, w)} .
$$

Moreover, if we assume that $w \in A_{\infty} \cap \mathcal{B}_{p, q}$, then for $f \in L^{0}\left(\mathbb{R}^{n}\right)$, we have that

$$
\left\|M_{\lambda}^{\sharp, d} f\right\|_{\mathcal{M}_{q}^{p}(d x, w)} \lesssim \lambda, p, q, w\|f\|_{\mathcal{M}_{q}^{p}(d x, w)} .
$$

Proof. First, we observe that for any $\eta>0$,

$$
M_{\lambda}^{\sharp, d} f(x) \lesssim_{\lambda, \eta} M^{(\eta)} f(x) .
$$

To see this, let $Q \in \mathcal{Q}$ and $m_{f}(Q)$ be any median. We see that by (33),

$$
\begin{aligned}
\omega_{\lambda}(f ; Q) & \leq\left(\left(f-m_{f}(Q)\right) \chi_{Q}\right)^{*}(\lambda|Q|) \\
& \leq\left(f \cdot \chi_{Q}\right)^{*}\left(\frac{\lambda}{2}|Q|\right)+\left(m_{f}(Q) \chi_{Q}\right)^{*}\left(\frac{\lambda}{2}|Q|\right) \leq 2\left(f \cdot \chi_{Q}\right)^{*}\left(\frac{\lambda}{2}|Q|\right) .
\end{aligned}
$$

Moreover, we notice that

$$
\begin{aligned}
\left(f \cdot \chi_{Q}\right)^{*}\left(\frac{\lambda}{2}|Q|\right) & \leq\left(\frac{1}{\lambda / 2 \cdot|Q|} \int_{0}^{\frac{\lambda}{2}|Q|}\left(f \cdot \chi_{Q}\right)^{*}(t)^{\eta} d t\right)^{\frac{1}{\eta}} \\
& \leq\left(\frac{1}{\lambda / 2 \cdot|Q|} \int_{Q}|f(x)|^{\eta} d x\right)^{\frac{1}{\eta}} .
\end{aligned}
$$

In summary, we conclude that

$$
\begin{aligned}
M_{\lambda}^{\sharp, d} f(x) & \leq 2 \sup _{Q_{0} \in \mathcal{Q}} \sup _{Q \in \mathcal{D}\left(Q_{0}\right)}\left(f \cdot \chi_{Q}\right)^{*}\left(\frac{\lambda}{2}|Q|\right) \chi_{Q}(x) \\
& \leq 2^{1+\frac{1}{\eta}} \lambda^{-\frac{1}{\eta}} \sup _{Q_{0} \in \mathcal{Q}} \sup _{Q \in \mathcal{D}\left(Q_{0}\right)}\left(\frac{1}{|Q|} \int_{Q}|f(x)|^{\eta} d x\right)^{\frac{1}{\eta}} \chi_{Q}(x),
\end{aligned}
$$

which implies (45). Next, we choose $u \in(1, \infty)$ such that $w \in A_{u}$ and $\eta:=\frac{q}{u}$. In view of (45) and Theorem 1.2, it follows that

$$
\left\|M_{\lambda}^{\sharp, d} f\right\|_{\mathcal{M}_{q}^{p}(w, w)} \lesssim_{\lambda, \eta}\left\|M^{(\eta)} f\right\|_{\mathcal{M}_{q}^{p}(w, w)} \lesssim_{p, q, w}\|f\|_{\mathcal{M}_{q}^{p}(w, w)},
$$

which proves (43).

Meanwhile, if we observe that $w \in \mathcal{B}_{p, q}$ is equivalent to $w \in \mathcal{B}_{\frac{p}{\eta}}, \frac{q}{\eta}$; see [31, then by (45) and Theorem 1.2, it follows that

$$
\left\|M_{\lambda}^{\sharp, d} f\right\|_{\mathcal{M}_{q}^{p}(d x, w)} \lesssim_{\lambda, \eta}\left\|M^{(\eta)} f\right\|_{\mathcal{M}_{q}^{p}(d x, w)} \lesssim p, q, w\|f\|_{\mathcal{M}_{q}^{p}(d x, w)},
$$

which proves (44).

By combining (15), (16), Corollary 4.3 and Proposition 4.4, we obtain Theorem 1.10, Next, let us prove Theorem 1.11. We will employ a similar method to the proof of Theorem 4.2 . 
Proof of Theorem 1.11. First, let us show that

$$
\|f\|_{\mathcal{M}_{s}^{p}(w, w)} \lesssim_{p, s, w}\left\|M_{\lambda_{w}}^{\sharp, d} f\right\|_{\mathcal{M}_{s}^{p}(w, w)}
$$

by assuming $\lim _{l \rightarrow \infty} m_{f}\left(2^{l} Q_{0}\right) \rightarrow 0$ for all $Q_{0} \in \mathcal{Q}$. To this end, we fix any $Q_{0} \in \mathcal{Q}$ and calculate that

$$
\begin{gathered}
w\left(Q_{0}\right)^{\frac{1}{p}-\frac{1}{s}}\left(\int_{Q_{0}}|f(x)|^{s} d w(x)\right)^{\frac{1}{s}} \lesssim_{s} w\left(Q_{0}\right)^{\frac{1}{p}-\frac{1}{s}}\left(\int_{Q_{0}}\left|f(x)-m_{f}\left(2^{l} Q_{0}\right)\right|^{s} d w(x)\right)^{\frac{1}{s}} \\
+w\left(Q_{0}\right)^{\frac{1}{p}}\left|m_{f}\left(2^{l} Q_{0}\right)\right| .
\end{gathered}
$$

By the assumption, it follows that

$$
w\left(Q_{0}\right)^{\frac{1}{p}-\frac{1}{s}}\left(\int_{Q_{0}}|f(x)|^{s} d w(x)\right)^{\frac{1}{s}} \lesssim_{l \rightarrow \infty} w\left(Q_{0}\right)^{\frac{1}{p}-\frac{1}{s}}\left(\int_{Q_{0}}\left|f(x)-m_{f}\left(2^{l} Q_{0}\right)\right|^{s} d w(x)\right)^{\frac{1}{s}}
$$

and hence, we focus on the quantity: $\left(\int_{Q_{0}}\left|f(x)-m_{f}\left(2^{l} Q_{0}\right)\right|^{s} d w(x)\right)^{\frac{1}{s}}$. We employ Proposition 4.1 again to decompose $f-m_{f}\left(2^{l} Q_{0}\right)$ and obtain that

$$
\left(\int_{Q_{0}}\left|f(x)-m_{f}\left(2^{l} Q_{0}\right)\right|^{s} d w(x)\right)^{\frac{1}{s}} \lesssim_{s}\left(\int_{Q_{0}} M_{\lambda_{w}}^{\sharp d} f(x)^{s} d w(x)\right)^{\frac{1}{s}}+\mathrm{I},
$$

where we defined

$$
\mathrm{I}:=\left[\int_{Q_{0}}\left(\sum_{k \in \mathbb{N}_{0}} \sum_{j \in J_{k}} \omega_{\lambda_{w}}\left(f ; Q_{j}^{k}\right) \chi_{Q_{j}^{k}}(x)\right)^{s} d w(x)\right]^{\frac{1}{s}} .
$$

Here, we remark that the family $\left\{Q_{j}^{k}\right\}_{k \in \mathbb{N}_{0}, j \in J_{k}} \subset \mathcal{D}\left(2^{l} Q_{0}\right)$ is a $w$-sparse family generated by $2^{l} Q_{0}$. To evaluate I, by considering the suitable dyadic setting, we have only to calculate the following two terms intrinsically:

$$
\begin{aligned}
\mathrm{I}_{a} & :=\left[\int_{Q_{0}}\left(\sum_{k \in \mathbb{N}_{0}} \sum_{\substack{j \in J_{k}: \\
Q_{j}^{k} \subset Q_{0}}} \omega_{\lambda_{w}}\left(f ; Q_{j}^{k}\right) \chi_{Q_{j}^{k}}(x)\right)^{s} d w(x)\right]^{\frac{1}{s}}, \\
\mathrm{I}_{b} & :=\left[\int_{Q_{0}}\left(\sum_{k \in \mathbb{N}_{0}} \sum_{\substack{j \in J_{k}: \\
Q_{j}^{k} \supsetneq Q_{0}}} \omega_{\lambda_{w}}\left(f ; Q_{j}^{k}\right) \chi_{Q_{j}^{k}}(x)\right]^{\frac{1}{s}} d w(x)\right]^{s} .
\end{aligned}
$$

For $\mathrm{I}_{a}$, we employ the same method used in the proof of Theorem 4.2, namely, by observing $\chi_{Q_{j}^{k}} \leq C_{w} M_{w}\left[\chi_{Q_{j}^{k} \cap \Omega_{k+1}^{c}}\right]$ and using the boundedness of $M_{w}$, it follows that

$$
\mathrm{I}_{a} \lesssim_{s, w}\left[\int_{Q_{0}}\left(\sum_{k \in \mathbb{N}_{0}} \sum_{\substack{j \in J_{k}: \\ Q_{j}^{k} \subset Q_{0}}} \omega_{\lambda_{w}}\left(f ; Q_{j}^{k}\right) \chi_{Q_{j}^{k} \cap \Omega_{k+1}^{c}}(x)\right)^{s} d w(x)\right]^{\frac{1}{s}} .
$$

If we notice that the summation is taken over the cubes contained in $Q_{0}$, then the disjointness of $\left\{Q_{j}^{k} \cap \Omega_{k+1}^{c}\right\}_{k \in \mathbb{N}_{0}, j \in J_{k}}$ yields that

$$
\mathrm{I}_{a} \lesssim_{s, w}\left(\int_{Q_{0}} M_{\lambda_{w}}^{\sharp, d} f(x)^{s} d w(x)\right)^{\frac{1}{s}} .
$$


Meanwhile, for $\mathrm{I}_{b}$, by recalling that $Q_{j}^{k} \subset 2^{l} Q_{0}$ and the dyadic property, we can rewrite the summation of $\mathrm{I}_{b}$ as follows:

$$
\mathrm{I}_{b} \leq\left[\int_{Q_{0}}\left(\sum_{m=1}^{l} \omega_{\lambda_{w}}\left(f ; Q_{0}^{(m)}\right) \chi_{Q_{0}^{(m)}}(x)\right)^{s} d w(x)\right]^{\frac{1}{s}} .
$$

Here, $Q_{0}^{(m)}$ denotes the dyadic $m$-th ancestor of $Q_{0}$, that is, $Q_{0}^{(m)}$ is a unique dyadic cube with respect to $2^{l} Q_{0}$ whose side length is $2^{m} \ell\left(Q_{0}\right)$ and containing $Q_{0}$. With the relation: $Q_{0} \subset Q_{0}^{(m)}$ in mind, we see that

$$
\begin{aligned}
\mathrm{I}_{b} & \leq w\left(Q_{0}\right)^{\frac{1}{s}} \sum_{m=1}^{l} \omega_{\lambda_{w}}\left(f ; Q_{0}^{(m)}\right) \\
& =w\left(Q_{0}\right)^{\frac{1}{s}} \sum_{m=1}^{l} \frac{1}{w\left(Q_{0}^{(m)}\right)^{\frac{1}{s}}}\left(\int_{Q_{0}^{(m)}} \omega_{\lambda_{w}}\left(f ; Q_{0}^{(m)}\right)^{s} d w(x)\right)^{\frac{1}{s}} \\
& \leq w\left(Q_{0}\right)^{\frac{1}{s}} \sum_{m=1}^{l} \frac{1}{w\left(Q_{0}^{(m)}\right)^{\frac{1}{p}}}\left\|M_{\lambda_{w}}^{\sharp d} f\right\|_{\mathcal{M}_{s}^{p}(w, w)} .
\end{aligned}
$$

Now, let us show that

$$
\sum_{m=1}^{l} \frac{1}{w\left(Q_{0}^{(m)}\right)^{\frac{1}{p}}} \lesssim_{p, w} \frac{1}{w\left(Q_{0}\right)^{\frac{1}{p}}} .
$$

Since $w \in A_{\infty}$, there exist $L_{w} \in \mathbb{N}$ and $\alpha_{w}>1$ such that

$$
w\left(Q_{0}^{\left(i+L_{w}\right)}\right) \geq \alpha_{w} w\left(Q_{0}^{(i)}\right), \quad\left(i \in \mathbb{N}_{0}, Q_{0} \in \mathcal{D}\right) .
$$

For the detail of this property, see Appendix. In particular, for $i, j \in \mathbb{N}$, it holds that

$$
w\left(Q_{0}^{\left(i+j L_{w}\right)}\right) \geq \alpha_{w} w\left(Q_{0}^{\left(i+(j-1) L_{w}\right)}\right) \geq \cdots \geq \alpha_{w}^{j} w\left(Q_{0}^{(i)}\right) .
$$

Hence, the left-hand side of (50) can be controlled as follows:

$$
\sum_{m=1}^{l} \frac{1}{w\left(Q_{0}^{(m)}\right)^{\frac{1}{p}}} \leq \sum_{i=1}^{L_{w}} \sum_{j=0}^{\left[l / L_{w}\right]+1} \frac{1}{w\left(Q_{0}^{\left(i+j L_{w}\right)}\right)^{\frac{1}{p}}} \leq \sum_{i=1}^{L_{w}} \frac{1}{w\left(Q_{0}^{(i)}\right)^{\frac{1}{p}}} \sum_{j=0}^{\left[l / L_{w}\right]+1} \frac{1}{\alpha_{w}^{\frac{j}{p}}} .
$$

If we recall that $w\left(Q_{0}\right) \leq w\left(Q_{0}^{(i)}\right)$ for any $i \in \mathbb{N}$ and that $\alpha_{w}>1$, then we see that

$$
\sum_{m=1}^{l} \frac{1}{w\left(Q_{0}^{(m)}\right)^{\frac{1}{p}}} \lesssim_{p, w} \frac{L_{w}}{w\left(Q_{0}\right)^{\frac{1}{p}}},
$$

which implies (50), since the constant $L_{w}$ and $\alpha_{w}$ depend only on $w$ (and dimension $n$ ). Remark that the implicit constant in (50) does not depend on $l \in \mathbb{N}$ as well. Therefore, we obtain the estimate of $\mathrm{I}_{b}$ :

$$
\mathrm{I}_{b} \lesssim p, w w\left(Q_{0}\right)^{-\frac{1}{p}+\frac{1}{s}}\left\|M_{\lambda_{w}}^{\sharp, d} f\right\|_{\mathcal{M}_{s}^{p}(w, w)} .
$$

In total, it follows from (48), (49) and (52) that

$$
\begin{aligned}
\left(\int_{Q_{0}}\left|f(x)-m_{f}\left(2^{l} Q_{0}\right)\right|^{s} d w(x)\right)^{\frac{1}{s}} \lesssim_{p, s, w} & \left(\int_{Q_{0}} M_{\lambda_{w}}^{\sharp, d} f(x)^{s} d w(x)\right)^{\frac{1}{s}} \\
& +w\left(Q_{0}\right)^{-\frac{1}{p}+\frac{1}{s}}\left\|M_{\lambda_{w}}^{\sharp, d} f\right\|_{\mathcal{M}_{s}^{p}(w, w)}
\end{aligned}
$$

and hence, in view of (47), we conclude (46).

Next, assuming the weighted integral condition (2) for $p, q$ and $w$, we prove

$$
\|f\|_{\mathcal{M}_{q}^{p}(d x, w)} \lesssim_{p, q, w}\left\|M_{\lambda_{w}^{\sharp}}^{\sharp, d} f\right\|_{\mathcal{M}_{q}^{p}(d x, w)} .
$$


Another inequality:

$$
\sup _{Q \in \mathcal{Q}} \Phi_{p, q, w}(Q)\left(\frac{1}{w(Q)} \int_{Q}|f(x)|^{s} d w(x)\right)^{\frac{1}{s}} \leq\|f\|_{\mathcal{M}_{q}^{p}(d x, w)}
$$

follows from Hölder's inequality directly. We need only mimic the above proof. Fix any $Q_{0} \in \mathcal{D}$ and calculate that

$$
\left|Q_{0}\right|^{\frac{1}{p}-\frac{1}{q}}\left(\int_{Q_{0}}|f(x)|^{q} d w(x)\right)^{\frac{1}{q}} \lesssim q \varlimsup_{l \rightarrow \infty}\left|Q_{0}\right|^{\frac{1}{p}-\frac{1}{q}}\left(\int_{Q_{0}}\left|f(x)-m_{f}\left(2^{l} Q_{0}\right)\right|^{q} d w(x)\right)^{\frac{1}{q}} .
$$

Again, Proposition 4.1, Lerner's decomposition formula reduces the matters to show the inequality: $\mathrm{II}_{a}+\mathrm{II}_{b} \lesssim_{p, q, w}\left\|M_{\lambda_{w}}^{\sharp, d} f\right\|_{\mathcal{M}_{q}^{p}(d x, w)}$, where,

$$
\begin{aligned}
& \mathrm{II}_{a}:=\left|Q_{0}\right|^{\frac{1}{p}-\frac{1}{q}}\left[\int_{Q_{0}}\left(\sum_{k \in \mathbb{N}_{0}} \sum_{\substack{j \in J_{k}: \\
Q_{j}^{k} \subset Q_{0}}} \omega_{\lambda_{w}}\left(f ; Q_{j}^{k}\right) \chi_{Q_{j}^{k}}(x)\right)^{s} d w(x)\right]^{\frac{1}{s}},
\end{aligned}
$$

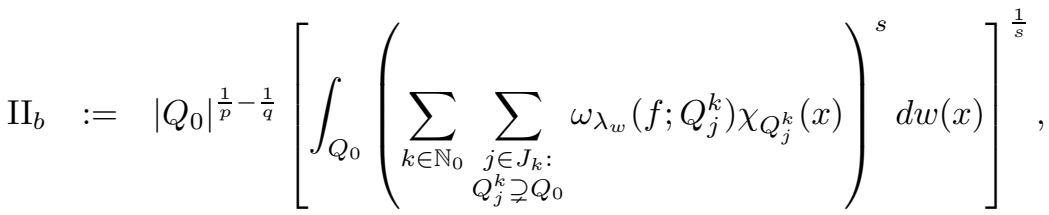

and $\left\{Q_{j}^{k}\right\}_{k \in \mathbb{N}_{0}, j \in J_{k}} \subset \mathcal{D}\left(2^{l} Q_{0}\right)$ is a $w$-sparse family generated by $2^{l} Q_{0}$. For $\mathrm{II}_{a}$, we may apply the same argument used to evaluate $\mathrm{I}_{a}$; see (49) to obtain that

$$
\mathrm{II}_{a} \lesssim_{q, w}\left|Q_{0}\right|^{\frac{1}{p}-\frac{1}{q}}\left(\int_{Q_{0}} M_{\lambda_{w}}^{\sharp, d} f(x)^{q} d w(x)\right)^{\frac{1}{q}} \leq\left\|M_{\lambda_{w}}^{\sharp, d} f\right\|_{\mathcal{M}_{q}^{p}(d x, w)} .
$$

For $\mathrm{II}_{b}$, we employ the weighted integral condition (2) for $p, q$ and $w$ instead of the $A_{\infty}$ condition. As before, using the dyadic $m$-ancestor of $Q_{0}$, we see that

$$
\begin{aligned}
\mathrm{II}_{b} & \leq\left|Q_{0}\right|^{\frac{1}{p}-\frac{1}{q}}\left(\int_{Q_{0}}\left(\sum_{m=1}^{l} \omega_{\lambda_{w}}\left(f ; Q_{0}^{(m)}\right) \chi_{Q_{0}^{(m)}}(x)\right)^{q} d w(x)\right)^{\frac{1}{q}} \\
& =\Phi_{p, q, w}\left(Q_{0}\right) \sum_{m=1}^{l} \omega_{\lambda_{w}}\left(f ; Q_{0}^{(m)}\right) \\
& \leq \Phi_{p, q, w}\left(Q_{0}\right) \sum_{m=1}^{l} \frac{1}{w\left(Q_{0}^{(m)}\right)^{\frac{1}{q}}}\left(\int_{Q_{0}^{(m)}} M_{\lambda_{w}}^{\sharp, d} f(x)^{q} d w(x)\right)^{\frac{1}{q}} .
\end{aligned}
$$

From the definition of the norm of $\mathcal{M}_{q}^{p}(d x, w)$, and the weighted integral condition (2) it follows that

$$
\mathrm{II}_{b} \leq \Phi_{p, q, w}\left(Q_{0}\right) \sum_{m=1}^{l} \frac{1}{\Phi_{p, q, w}\left(Q_{0}^{(m)}\right)}\left\|M_{\lambda}^{\sharp, d} f\right\|_{\mathcal{M}_{q}^{p}(d x, w)} \lesssim p, q, w\left\|M_{\lambda}^{\sharp, d} f\right\|_{\mathcal{M}_{q}^{p}(d x, w)} .
$$

By invoking Lemma 2.6 in Chapter 2 and (13), we obtain the direct analogy from original type of the sharp maximal inequality :

Corollary 4.5. Let $0<q \leq p<\infty$ and $w \in A_{\infty}$. 
(1) (Komori-Shirai type) For any $f \in L^{0}\left(\mathbb{R}^{n}\right)$ satisfying $M f \in \mathcal{M}_{q_{0}}^{p_{0}}\left(w_{0}, w_{0}\right)$ for some $0<q_{0} \leq p_{0}<\infty$ and $w_{0} \in A_{\infty}$, we have that

$$
\|f\|_{\mathcal{M}_{q}^{p}(w, w)} \sim_{p, q, w}\left\|M_{\lambda_{w}}^{\sharp, d} f\right\|_{\mathcal{M}_{q}^{p}(w, w)} \lesssim \lambda_{w}\left\|f^{\sharp}\right\|_{\mathcal{M}_{q}^{p}(w, w)} .
$$

(2) (Samko type) For any $f \in L^{0}\left(\mathbb{R}^{n}\right)$ satisfying $M f \in \mathcal{M}_{q_{0}}^{p_{0}}\left(d x, w_{0}\right)$ for some $0<q_{0} \leq$ $p_{0}<\infty$ and $w_{0}$ satisfying the weighted integral condition (2) for $p_{0}, q_{0}$ and $w_{0}$, we have that

$$
\|f\|_{\mathcal{M}_{q}^{p}(d x, w)} \sim_{p, q, w}\left\|M_{\lambda_{w}}^{\sharp, d} f\right\|_{\mathcal{M}_{q}^{p}(d x, w)} \lesssim \lambda_{w}\left\|f^{\sharp}\right\|_{\mathcal{M}_{q}^{p}(d x, w)} .
$$

Let us compare the above results with some recent researches. Sawano and Tanaka in 37. proved the following refinement of sharp maximal inequality:

Theorem 4.6. [37, Theorem 1.3] Let $1<q \leq p<\infty$. Then for any $f \in L^{0}\left(\mathbb{R}^{n}\right)$, we have that

$$
\|M f\|_{\mathcal{M}_{q}^{p}(d x, d x)} \lesssim_{p, q}\left\|f^{\sharp}\right\|_{\mathcal{M}_{q}^{p}(d x, d x)}+\|f\|_{\mathcal{M}_{1}^{p}(d x, d x)} .
$$

In view of (13) and Theorem 1.2 Theorem 1.10 improves and generalizes Theorem 4.6 Meanwhile, there exists a weighted result for the sharp maximal inequality obtained by KomoriFuruya in [22]:

Theorem 4.7. [22, Theorem 12] Let $1<q_{0} \leq q \leq p<\infty$ and $w \in A_{\infty}$. Then for $f \in L^{0}\left(\mathbb{R}^{n}\right)$ satisfying $M f \in \mathcal{M}_{q_{0}}^{p}(w, w)$, it holds that

$$
\|f\|_{\mathcal{M}_{q}^{p}(w, w)} \lesssim p, q, w\left\|f^{\sharp}\right\|_{\mathcal{M}_{q}^{p}(w, w)} .
$$

We can see easily that Corollaries 1.13 and 4.5 improve and generalize Theorem 4.7.

\section{Proof of Theorems 1.17 And 1.21 including ANOTher APPlication}

We first show Theorem 1.17. Since the assertion 1 of Theorem 1.17 is contained in Theorem 1.21, we prove only the assertion 2 of Theorem 1.17 here.

Proof of Theorem 1.172-(a). Assume that $R_{i}$ is bounded on $\mathcal{M}_{q}^{p}(d x, w)$ for some $i=1, \ldots, n$. To show (7), we fix any $Q \in \mathcal{Q}$ and take any $f \geq 0$ such that $f \cdot \chi_{Q} \in \mathcal{M}_{q}^{p}(d x, w)$. We focus on the cube $\widetilde{Q}:=Q+2 \ell(Q) e_{i}$. Here, $e_{i}$ denotes the $i$-th elementary vector. If we notice that for any $x \in \widetilde{Q}$, it holds that $x_{i}-y_{i} \geq \ell(Q)$ and that $|x-y| \leq C \ell(Q)$, then we have the pointwise estimate

$$
\left|R_{i}\left[f \cdot \chi_{Q}\right](x)\right|=\int_{Q} f(y) \frac{x_{i}-y_{i}}{|x-y|^{n+1}} d y \geq C \frac{1}{|Q|} \int_{Q} f(y) d y .
$$

Hence it follows from the boundedness of $R_{i}$ that

$$
\frac{1}{|Q|} \int_{Q} f(y) d y \leq \frac{C}{\left\|\chi_{\widetilde{Q}}\right\|_{\mathcal{M}_{q}^{p}(d x, w)}}\left\|R_{i}\left[f \cdot \chi_{Q}\right] \chi_{\widetilde{Q}}\right\|_{\mathcal{M}_{q}^{p}(d x, w)} \leq C \frac{\left\|f \cdot \chi_{Q}\right\|_{\mathcal{M}_{q}^{p}(d x, w)}}{\left\|\chi_{\widetilde{Q}}\right\|_{\mathcal{M}_{q}^{p}(d x, w)}} .
$$

Namely, we have that for any $f \geq 0$ such that $f \cdot \chi_{Q} \in \mathcal{M}_{q}^{p}(d x, w)$,

$$
\frac{1}{|Q|} \int_{Q} f(y) d y \cdot\left\|\chi_{\widetilde{Q}}\right\|_{\mathcal{M}_{q}^{p}(d x, w)} \leq C\left\|f \cdot \chi_{Q}\right\|_{\mathcal{M}_{q}^{p}(d x, w)}
$$

Now, we take any $g \geq 0$ such that $\left\|g \cdot \chi_{\widetilde{Q}}\right\|_{\mathcal{M}_{q}^{p}(d x, w)} \leq 1$ and put $f=\left|R_{i}\left[g \cdot \chi_{\widetilde{Q}}\right]\right|$ as in (154) to obtain that

$$
\frac{1}{|Q|} \int_{Q}\left|R_{i}\left[g \cdot \chi_{\widetilde{Q}}\right](x)\right| d x \cdot\left\|\chi_{\widetilde{Q}}\right\|_{\mathcal{M}_{q}^{p}(d x, w)} \leq C\left\|g \cdot \chi_{\widetilde{Q}}\right\|_{\mathcal{M}_{q}^{p}(d x, w)} \leq C .
$$


Here, we again used the boundedness of $R_{i}$. Meanwhile, if we go through a similar argument as before, we notice

$$
\frac{1}{|\widetilde{Q}|} \int_{\widetilde{Q}} g(y) d y \leq C \inf _{x \in Q}\left|R_{i}\left[g \cdot \chi_{\widetilde{Q}}\right](x)\right| \leq C \frac{1}{|Q|} \int_{Q}\left|R_{i}\left[g \cdot \chi_{\widetilde{Q}}\right](x)\right| d x .
$$

By combining (55) and (56), it follows that for any $g \geq 0$ such that $\left\|g \cdot \chi_{\widetilde{Q}}\right\|_{\mathcal{M}_{q}^{p}(d x, w)} \leq 1$,

$$
\frac{1}{|\widetilde{Q}|} \int_{\widetilde{Q}} g(y) d y \cdot\left\|\chi_{\widetilde{Q}}\right\|_{\mathcal{M}_{q}^{p}(d x, w)} \leq C .
$$

Moreover using (6), we see that

$$
\frac{1}{|\widetilde{Q}|}\left\|\chi_{\widetilde{Q}}\right\|_{\mathcal{M}_{q}^{p}(d x, w)}\left\|w^{-\frac{1}{q}} \chi_{\widetilde{Q}}\right\|_{H^{q^{\prime}, n(1-q / p)}}=\frac{\left\|\chi_{\widetilde{Q}}\right\|_{\mathcal{M}_{q}^{p}(d x, w)}}{|\widetilde{Q}|} \sup _{\substack{g \geq 0: \\\left\|g \chi_{\widetilde{Q}}\right\|_{\mathcal{M}_{q}^{p}(d x, w)} \leq 1}} \int_{\widetilde{Q}} g(y) d y \leq C,
$$

which implies (7).

Next, we prove the weighted integral condition (2) for $p, q$ and $w$ by assuming that $w \in \mathcal{B}_{p, q}$ satisfies the doubling condition and that some genuine singular integral operator $T$ is bounded on $\mathcal{M}_{q}^{p}(d x, w)$. To this end, we recall the equivalent condition of the weighted integral condition (2) and the genuine singular integral operator introduced in [3].

Lemma 5.1 ([30]). Let $0<q \leq p<\infty$ and $w$ satisfies the doubling condition. Then the following are equivalent:

(1) The weighted integral condition for $p, q$ and $w$ holds.

(2) There exists $c>1$ such that

$$
2 \Phi_{p, q, w}(Q) \leq \Phi_{p, q, w}(c Q)
$$

for any $Q \in \mathcal{Q}$.

The idea of the following proof goes back to [11, Theorem 6.9].

Proof of Theorem 1.172-(b). By assuming that the weighted integral condition (2) for $p, q$ and $w$ fails to hold, let us obtain the contradiction. With Lemma 5.1 in mind, we assume that for any $m \in \mathbb{N}$, there exists $Q_{m} \in \mathcal{Q}$ such that $2 \Phi_{p, q, w}\left(Q_{m}\right)>\Phi_{p, q, w}\left(2^{m} Q_{m}\right)$. We take the genuine singular integral operator $T$ and denote the cone $V_{\theta, R}$ by $V$. Set

$$
f_{m}(y):=\chi_{V-c\left(Q_{m}\right)}(-y) \chi_{2^{m-1} Q_{m} \backslash 2^{6} Q_{m}}(y),
$$

for $m \geq 7$. Since $V$ is a cone, we may take another cube $R_{m}$ so that $R_{m} \subset\left(V+c\left(Q_{m}\right)\right) \cap$ $\left(2^{4} Q_{m} \backslash Q_{m}\right), Q_{m} \subset C_{V} R_{m}$ for some $C_{V}>0$ and that $\left|R_{m}\right| \sim_{V}\left|Q_{m}\right|$. Note that for any $x \in R_{m}$ and any $y \in 2^{m-1} Q_{m} \backslash 2{ }^{6} Q_{m}$ such that $-y \in V-c\left(Q_{m}\right)$, we have $x-y \in V$ and $\ell\left(Q_{m}\right) \lesssim|x-y| \lesssim 2^{m} \ell\left(Q_{m}\right)$. Hence, it follows that for any $x \in R_{m}$,

$$
\begin{aligned}
T f_{m}(x) & =\int_{\mathbb{R}^{n}} K(x, y) \chi_{V-c\left(Q_{m}\right)}(-y) \chi_{2^{m-1} Q_{m} \backslash 2^{6} Q_{m}}(y) d y \\
& \sim \int_{\left\{x-y \in V: \ell\left(Q_{m}\right)<|x-y| \leq 2^{m} \ell\left(Q_{m}\right)\right\}} K(x, y) f_{m}(y) d y \\
& \geq C \int_{\left\{\ell\left(Q_{m}\right)<|x-y| \leq 2^{m} \ell\left(Q_{m}\right)\right\}} \frac{d y}{|x-y|^{n}} \\
& \sim m .
\end{aligned}
$$


This implies that $m \cdot \chi_{R_{m}}(x) \lesssim T f_{m}(x)$. Meanwhile, if we recall that $Q_{m} \subset C_{V} R_{m},\left|Q_{m}\right| \sim\left|R_{m}\right|$ and $w$ is a doubling weight, then we see that

$$
\Phi_{p, q, w}\left(Q_{m}\right) \lesssim\left|R_{m}\right|^{\frac{1}{p}}\left(\frac{w\left(C_{V} R_{m}\right)}{\left|R_{m}\right|}\right)^{\frac{1}{q}} \lesssim \Phi_{p, q, w}\left(R_{m}\right) \leq\left\|\chi_{R_{m}}\right\|_{\mathcal{M}_{q}^{p}(d x, w)} .
$$

Hence, the boundedness of $T$ yields

$$
\Phi_{p, q, w}\left(Q_{m}\right) \lesssim \frac{1}{m}\left\|T f_{m}\right\|_{\mathcal{M}_{q}^{p}(d x, w)} \lesssim \frac{1}{m}\left\|\chi_{2^{m} Q_{m}}\right\|_{\mathcal{M}_{q}^{p}(d x, w)} .
$$

Moreover, by $w \in \mathcal{B}_{p, q}$ and $\Phi_{p, q, w}\left(2^{m} Q_{m}\right)<2 \Phi_{p, q, w}\left(Q_{m}\right)$, we see that

$$
\Phi_{p, q, w}\left(Q_{m}\right) \lesssim \frac{1}{m} \Phi_{p, q, w}\left(2^{m} Q_{m}\right) \leq \frac{2}{m} \Phi_{p, q, w}\left(Q_{m}\right)
$$

which implies $1 \lesssim m^{-1}$ holds for any $m \in \mathbb{N}$. This is a contradiction.

Now, we prove Theorem 1.21 by assuming that $M$ is bounded on $\mathcal{M}_{q}^{p}(d x, w)$ and that the weighted integral condition (2) for $p, q$ and $w$. To this end, we first extend the definition of the commutator $[b, T]$ as a linear operator defined on $\mathcal{M}_{q}^{p}(d x, w)$. We remark that once we assume $w \in H L M_{q}^{p}$, then $w \in A_{\infty}$ automatically by Lemma 2.2. Let $b \in$ BMO and $T$ be a singular integral operator. Moreover, we take $\varepsilon \in(0,1)$ as in Corollary 2.9. For $f \in \mathcal{M}_{q}^{p}(d x, w)$ and $x \in \mathbb{R}^{n}$, we define

$$
[b, T] f(x):=[b, T]_{0}\left(f \cdot \chi_{2 Q}\right)(x)+\int_{\mathbb{R}^{n} \backslash 2 Q}(b(x)-b(y)) K(x, y) f(y) d y,
$$

where $Q$ is any cube containing the point $x$ and $[b, T]_{0}$ denotes the commutator as a bounded linear operator on $L^{\frac{1}{1-\varepsilon}}\left(\mathbb{R}^{n}\right)$. It is easy to check that the definition of $[b, T] f(x)$ does not depend on the choice of the cube $Q$ which contains $x$. In addition, since we know that $f \cdot \chi_{Q} \in L^{\frac{1}{1-\varepsilon}}\left(\mathbb{R}^{n}\right)$ for any $f \in \mathcal{M}_{q}^{p}(d x, w)$ and $Q \in \mathcal{Q}$ by Corollary [2.9, the first term: $[b, T]_{0}\left(f \cdot \chi_{2 Q}\right)(x)$ is well defined. For the second term, we have the following lemma:

Lemma 5.2. Let $1<q \leq p<\infty$ and $w \in H L M_{q}^{p}$ satisfy the weighted integral condition (2) for $p, q$ and $w$. Then for any $x \in \mathbb{R}^{n}$ and any $Q \in \mathcal{Q}$ containing $x$, we have that

$$
\int_{\mathbb{R}^{n} \backslash 2 Q}|(b(x)-b(y)) K(x, y) f(y)| d y \leq C \frac{\|f\|_{\mathcal{M}_{q}^{p}(d x, w)}}{\Phi_{p, q, w}(Q)}\left(\|b\|_{\mathrm{BMO}}+\left|b(x)-b_{Q}\right|\right),
$$

where $b_{Q}$ denotes the mean value of $b$ on $Q$ with respect to the Lebesgue measure, that is, $b_{Q}:=\frac{1}{|Q|} \int_{Q} b(x) d x$.

Proof. It follows from the size condition of the kernel $K$ that

$$
\int_{\mathbb{R}^{n} \backslash 2 Q}|(b(x)-b(y)) K(x, y) f(y)| d y \leq C(\mathrm{I}+\mathrm{II}),
$$

where we denined

$$
\mathrm{I}:=\int_{\mathbb{R}^{n} \backslash 2 Q} \frac{\left|b(x)-b_{Q}\right|}{|x-y|^{n}}|f(y)| d y, \quad \text { II }:=\int_{\mathbb{R}^{n} \backslash 2 Q} \frac{\left|b(y)-b_{Q}\right|}{|x-y|^{n}}|f(y)| d y .
$$

We first evaluate the second term II. Since we assume the boundedness of $M$ on $\mathcal{M}_{q}^{p}(d x, w)$, we can choose $\varepsilon \in(0,1)$ such that $M^{\left(\frac{1}{1-\varepsilon}\right)}$ is also bounded on $\mathcal{M}_{q}^{p}(d x, w)$ by Lemma 2.8, Then 
we have

$$
\begin{aligned}
\mathrm{II} & \leq \sum_{l=1}^{\infty} \frac{C}{\left|2^{l} Q\right|} \int_{2^{l} Q}\left|b(y)-b_{Q}\right||f(y)| d y \\
& \leq C \sum_{l=1}^{\infty}\left(\frac{1}{\left|2^{l} Q\right|} \int_{2^{l} Q}\left|b(y)-b_{Q^{\mid}}\right|^{\frac{1}{\varepsilon}} d y\right)^{\varepsilon}\left(\frac{1}{\left|2^{l} Q\right|} \int_{2^{l} Q}|f(y)|^{\frac{1}{1-\varepsilon}} d y\right)^{1-\varepsilon} \\
& \leq C \sum_{l=1}^{\infty}\left(\frac{1}{\left|2^{l} Q\right|} \int_{2^{l} Q}\left|b(y)-b_{Q}\right|^{\frac{1}{\varepsilon}} d y\right)^{\varepsilon} \frac{\left\|\chi_{2^{l} Q}\right\|_{\mathcal{M}_{q}^{p}(d x, w)}}{\Phi_{p, q, w}\left(2^{l} Q\right)} \inf _{x \in 2^{l} Q} M^{\left(\frac{1}{1-\varepsilon}\right)} f(x) \\
& \leq C \sum_{l=1}^{\infty}\left(\frac{1}{\left|2^{l} Q\right|} \int_{2^{l} Q}\left|b(y)-b_{Q}\right|^{\frac{1}{\varepsilon}} d y\right)^{\varepsilon} \frac{\|f\|_{\mathcal{M}_{q}^{p}(d x, w)}}{\Phi_{p, q, w}\left(2^{l} Q\right)}
\end{aligned}
$$

Now, we focus on the term related to the function $b$. It is easy to see that $\left|b_{2^{l} Q}-b_{Q}\right| \leq$ $C l\|b\|_{\text {BMo. Hence, we have that }}$

$$
\begin{aligned}
\left(\frac{1}{\left|2^{l} Q\right|} \int_{2^{l} Q}\left|b(y)-b_{Q}\right|^{\frac{1}{\varepsilon}} d y\right)^{\varepsilon} & \leq\left(\frac{1}{\left|2^{l} Q\right|} \int_{2^{l} Q}\left|b(y)-b_{2^{l} Q}\right|^{\frac{1}{\varepsilon}} d y\right)^{\varepsilon}+\left|b_{2^{l} Q}-b_{Q}\right| \\
& \leq C(1+l)\|b\|_{\mathrm{BMO}}
\end{aligned}
$$

for all $l \in \mathbb{N}$. It thus follows from Lemma 2.4 that

$$
\mathrm{II} \leq C\|b\|_{\mathrm{BMO}}\|f\|_{\mathcal{M}_{q}^{p}(d x, w)} \sum_{l=1}^{\infty} \frac{l}{\Phi_{p, q, w}\left(2^{l} Q\right)} \leq C\|b\|_{\mathrm{BMO}} \frac{\|f\|_{\mathcal{M}_{q}^{p}(d x, w)}}{\Phi_{p, q, w}(Q)} .
$$

Next, we focus on the first term I. We employ (5), (7) and the weighted integral condition (2) for $p, q$ and $w$ to obtain that

$$
\begin{aligned}
I & \leq C\left|b(x)-b_{Q}\right|\left(\sum_{l=1}^{\infty} \frac{1}{\left|2^{l} Q\right|} \int_{2^{l} Q}|f(y)| d y\right) \\
& \leq C\left|b(x)-b_{Q}\right|\left(\sum_{l=1}^{\infty} \frac{1}{\left|2^{l} Q\right|}\left\|w^{-\frac{1}{q}} \chi_{2^{l} Q}\right\|_{H^{q^{\prime}, n(1-q) p}}\|f\|_{\mathcal{M}_{q}^{p}(d x, w)}\right) \\
& \leq C\left|b(x)-b_{Q}\right| \cdot\|f\|_{\mathcal{M}_{q}^{p}(d x, w)} \sum_{l=1}^{\infty} \frac{1}{\Phi_{p, q, w}\left(2^{l} Q\right)} \\
& \leq C\left|b(x)-b_{Q}\right| \frac{\|f\|_{\mathcal{M}_{q}^{p}(d x, w)}}{\Phi_{p, q, w}(Q)} .
\end{aligned}
$$

Hence, we complete the proof of Lemma 5.2 .

Additionally, we need the following simple observation.

Lemma 5.3. Let $0<q<\infty, w \in A_{\infty}$ and $b \in$ BMO. Then for any cube $Q$, we have that

$$
\left(\frac{1}{w(Q)} \int_{Q}\left|b(x)-b_{Q}\right|^{q} w(x) d x\right)^{\frac{1}{q}} \leq C\|b\|_{\mathrm{BMO}}
$$

Since the proof of Lemma 5.3 is fundamental, we postpone its proof till Appendix. Using Theorem 1.11 and Lemmas 5.2 and 5.3 , let us complete the proof of Theorem 1.21 . 
Proof of Theorem 1.21. Fix any $f \in \mathcal{M}_{q}^{p}(d x, w)$ and any cube $Q$ and set

$$
\begin{aligned}
\mathrm{I} & :=|Q|^{\frac{1}{p}-\frac{1}{q}}\left(\int_{Q}\left|[b, T]_{0}\left(f \cdot \chi_{2 Q}\right)(x)\right|^{q} w(x) d x\right)^{\frac{1}{q}}, \\
\mathrm{II} & :=|Q|^{\frac{1}{p}-\frac{1}{q}}\left(\int_{Q}\left|\int_{\mathbb{R}^{n} \backslash 2 Q}(b(x)-b(y)) K(x, y) f(y) d y\right|^{q} w(x) d x\right)^{\frac{1}{q}} .
\end{aligned}
$$

Here, $[b, T]_{0}$ denotes the commutator in the sense of a bounded linear operator on $L^{\frac{1}{1-\varepsilon}}\left(\mathbb{R}^{n}\right)$ as before. All we have to do is to show that I, II $\leq C\|f\|_{\mathcal{M}_{q}^{p}(d x, w)}$. For the first term, we notice that $[b, T]_{0}\left(f \cdot \chi_{2 Q}\right) \in L^{\frac{1}{1-\varepsilon}}\left(\mathbb{R}^{n}\right)$, particularly,

$$
\lim _{l \rightarrow \infty} m_{[b, T]_{0}\left(f \cdot \chi_{2 Q}\right)}\left(2^{l} Q\right)=0
$$

holds for all $Q \in \mathcal{Q}$ and all medians. Hence, we may apply Theorem 1.11 to obtain that

$$
\mathrm{I} \leq\left\|[b, T]_{0}\left(f \cdot \chi_{2 Q}\right)\right\|_{\mathcal{M}_{q}^{p}(d x, w)} \leq C\left\|\left\{[b, T]_{0}\left(f \cdot \chi_{2 Q}\right)\right\}^{\sharp}\right\|_{\mathcal{M}_{q}^{p}(d x, w)} .
$$

Since we have the pointwise estimate; see [10, Lemma 3.5.5]:

$$
\left\{[b, T]_{0}\left(f \cdot \chi_{2 Q}\right)\right\}^{\sharp}(x) \leq C\|b\|_{\mathrm{BMO}}\left(M^{(\eta)}\left[f \cdot \chi_{2 Q}\right](x)+M^{2}\left[f \cdot \chi_{2 Q}\right](x)\right)
$$

for any $\eta>1$, by taking $\eta:=\frac{1}{1-\varepsilon}>1$, it follows that

$$
\mathrm{I} \leq C\|b\|_{\mathrm{BMO}}\|f\|_{\mathcal{M}_{q}^{p}(d x, w)} .
$$

Here, we also used the boundedness of $M^{\left(\frac{1}{1-\varepsilon}\right)}$ on $\mathcal{M}_{q}^{p}(d x, w)$.

For the second term, we employ Lemma 5.2 to obtain that

$$
\begin{aligned}
\mathrm{II} & \leq C\left(\|b\|_{\mathrm{BMO}}\|f\|_{\mathcal{M}_{q}^{p}(d x, w)}+\frac{\|f\|_{\mathcal{M}_{q}^{p}(d x, w)}}{\Phi_{p, q, w}(Q)}|Q|^{\frac{1}{p}-\frac{1}{q}}\left(\int_{Q}\left|b(x)-b_{Q}\right|^{q} w(x) d x\right)^{\frac{1}{q}}\right) \\
& =C\left(\|b\|_{\mathrm{BMO}}\|f\|_{\mathcal{M}_{q}^{p}(d x, w)}+\|f\|_{\mathcal{M}_{q}^{p}(d x, w)}\left(\frac{1}{w(Q)} \int_{Q}\left|b(x)-b_{Q}\right|^{q} w(x) d x\right)^{\frac{1}{q}}\right) .
\end{aligned}
$$

By virtue of Lemma 5.3 , we see that II $\leq C\|b\|_{\mathrm{BMO}}\|f\|_{\mathcal{M}_{q}^{p}(d x, w)}$.

Here, we note the result of the commutators on weighted Morrey spaces $\mathcal{M}_{q}^{p}(w, w)$ of KomoriShirai type.

Theorem $5.4([23])$. Let $1<q \leq p<\infty, w \in A_{q}$.

(1) The singular integral operator $T$ is bounded on $\mathcal{M}_{q}^{p}(w, w)$.

(2) Assume that $b \in \mathrm{BMO}$. Then the commutator $[b, T]$ generated by a singular integral operator $T$ with respect to $b$ is bounded on $\mathcal{M}_{q}^{p}(w, w)$.

As another application, we consider the boundedness of $M$ on the pre-dual space of $\mathcal{M}_{q}^{p}(d x, w)$. In [25, Lerner connected the boundedness of maximal operator $M$ and the sharp maximal inequality in the framework of Banach function spaces. Here, we do not give the definitions of the Banach function space $X$ and its Köthe dual space $X^{\prime}$. We refer [2] for these definitions.

Proposition 5.5. 25, Corollary 4.3] Let $X$ be a Banach function space on which $M$ is bounded. Then $M$ is bounded on $X^{\prime}$ if and only if there exists $c>0$ such that for any $f \in L^{0}\left(\mathbb{R}^{n}\right)$ such that $\lim _{R \rightarrow \infty} f^{*}(R)=0$,

$$
\|f\|_{X} \leq c\left\|f^{\sharp}\right\|_{X}
$$


Unfortunately, the Morrey spaces are not Banach function spaces in general; see [40, Example 3.3]. Nevertheless, we still have an analogy of Proposition 5.5 in the framework of "ball Banach function spaces". We refer [40] for the motivation of the notion of ball Banach function spaces.

Let $\mathbb{M}^{+}$be the set of all nonnegative $d x$-measurable functions.

Definition 5.6. [40] A mapping $\rho: \mathbb{M}^{+} \rightarrow[0, \infty]$ is called a "ball Banach function norm" if, for all $f, g,\left\{f_{k}\right\}_{k \in \mathbb{N}}$, in $\mathbb{M}^{+}$, for all constants $a \geq 0$ and for all cubes $Q$ in $\mathbb{R}^{n}$, the following properties hold:

(P1) $\rho(f)=0 \Leftrightarrow f=0 d x$-a.e.; $\rho(a f)=a \rho(f) ; \rho(f+g) \leq \rho(f)+\rho(g)$;

(P2) $\rho(g) \leq \rho(f)$ if $0 \leq g \leq f d x$-a.e. ;

(P3) $\rho\left(f_{k}\right) \uparrow \rho(f)$ if $0 \leq f_{k} \uparrow f d x$-a.e. ;

$(\mathrm{P} 4)^{\prime} \rho\left(\chi_{Q}\right)<\infty$;

$(\mathrm{P} 5)^{\prime} \int_{Q} f(x) d x \leq C_{Q} \rho(f)$ with the constant $C_{Q}$ depending on $Q$ and $\rho$ but independent of $f$.

The difference between the Banach function spaces and the ball Banach function spaces appears in $(\mathrm{P} 4)^{\prime}$ and $(\mathrm{P} 5)^{\prime}$.

Under a certain condition on weights, $\mathcal{M}_{q}^{p}(d x, w)$ is a ball Banach function space when $1<q \leq p<\infty$.

Proposition 5.7. Let $1<q \leq p<\infty$ and $w \in H L M_{q}^{p}$. Then $\mathcal{M}_{q}^{p}(d x, w)$ is a ball Banach function space.

Proof. It suffices to check the conditions $(\mathrm{P} 4)^{\prime}$ and $(\mathrm{P} 5)^{\prime}$. Other conditions are trivial. Since $w \in H L M_{q}^{p}$, we know that $w \in \mathcal{B}_{p, q}$ and that (24) holds for all $Q \in \mathcal{Q}$ by Lemma 2.2. The condition $(\mathrm{P} 4)^{\prime}$ follows from $w \in \mathcal{B}_{p, q}$ directly. Indeed, $w \in \mathcal{B}_{p, q}$ implies $\left\|\chi_{Q}\right\|_{\mathcal{M}_{q}^{p}(d x, w)} \sim$ $\Phi_{p, q, w}(Q)<\infty$. The condition (P5)' follows from (23). In fact, we have

$$
\int_{Q}|f(x)| d x \leq C_{0} \frac{|Q|}{\Phi_{p, q, w}(Q)}\|f\|_{\mathcal{M}_{q}^{p}(d x, w)}
$$

Let $\mathbb{M}$ denote the collection of all extended scalar-valued (real or complex) $d x$-measurable functions on $\mathbb{R}^{n}$. Let $\rho$ be a ball Banach function norm. The collection $X=X(\rho)$ of all functions $f$ in $\mathbb{M}$ for which $\rho(|f|)<\infty$ is called a "ball Banach function space". For each $f \in X$, define $\|f\|_{X}:=\rho(|f|)$.

If $\rho$ is a ball Banach function norm, its "associated norm" $\rho^{\prime}$ is defined on $\mathbb{M}^{+}$by

$$
\rho^{\prime}(g):=\sup \left\{\int_{\mathbb{R}^{n}} f(x) g(x) d x: f \in \mathbb{M}^{+}, \rho(f) \leq 1\right\}, \quad\left(g \in \mathbb{M}^{+}\right) .
$$

Then the associated norm $\rho^{\prime}$ is itself a ball Banach function norm. The ball Banach function space $X\left(\rho^{\prime}\right)$ determined by $\rho^{\prime}$ is called the "associated space" of $X$ and is denoted by $X^{\prime}$.

Since the proof of Proposition 5.5 depends only on the properties (P1), (P2) and (P3), we can obtain the same result for the ball Banach function spaces. 
Proposition 5.8. Let $X$ be a ball Banach function space on which $M$ is bounded. Then $M$ is bounded on $X^{\prime}$ if and only if there exists $c>0$ such that for any $f \in L^{0}\left(\mathbb{R}^{n}\right)$ such that

$$
\lim _{R \rightarrow \infty} f^{*}(R)=0
$$

and that

$$
\|f\|_{X} \leq c\left\|f^{\sharp}\right\|_{X} .
$$

Remark 5.9. The assumption (58) in Proposition 5.8 implies (17) in Theorem 1.11. In fact, if one assumes (58), then we have that

$$
\left|m_{f}\left(2^{l} Q\right)\right| \leq\left(f \cdot \chi_{2^{l} Q}\right)^{*}\left(2^{-2}\left|2^{l} Q\right|\right) \leq f^{*}\left(2^{-2}\left|2^{l} Q\right|\right) \rightarrow 0 .
$$

As the example of $f$, where $f: \mathbb{R} \rightarrow \mathbb{R}$ is given by,

$$
f=\sum_{j=3}^{\infty} \chi_{[j !, j !+1]}
$$

shows that the assumption (58) is stronger than (17).

Thanks to Proposition 5.8 and Remark 5.9, the inequality (59) for any $f \in L^{0}\left(\mathbb{R}^{n}\right)$ satisfying (17) implies the boundedness of $M$ on $X^{\prime}$ follows automatically. Particularly, we proved (59) with $X=\mathcal{M}_{q}^{p}(d x, w)$ under the suitable conditions in Corollary 1.13. On the other hand, the second author and Tanaka obtained the characterization of the Köthe dual space of $\mathcal{M}_{q}^{p}(d x, w)$ in [39, 40]. For $\sigma(x)=w(x)^{-\frac{q^{\prime}}{q}}$, we denote all measurable functions $f$ for which norm

$$
\begin{aligned}
\|f\|_{H^{q^{\prime}, n(1-q / p)}(d x, \sigma)} & :=\left\|w^{-\frac{1}{q}} f\right\|_{H^{q^{\prime}, n(1-q / p)}} \\
& =\inf _{b \in \mathfrak{B}_{n(1-q / p)}}\left(\int_{\mathbb{R}^{n}}|f(x)|^{q^{\prime}} \sigma(x) b(x)^{-\frac{q^{\prime}}{q}} d x\right)^{\frac{1}{q^{\prime}}} \\
& =\inf _{b \in \mathfrak{B}_{n(1-q / p)}}\left(\int_{\mathbb{R}^{n}}|f(x)|^{q^{\prime}}(w(x) b(x))^{-\frac{q^{\prime}}{q}} d x\right)^{\frac{1}{q^{\prime}}}
\end{aligned}
$$

is finite by $H^{q^{\prime}, n(1-q / p)}(d x, \sigma)$. Then we have that $\mathcal{M}_{q}^{p}(d x, w)^{\prime}=H^{q^{\prime}, n(1-q / p)}(d x, \sigma)$ when $1<q \leq p<\infty$; see [39, 40]. As a result, we obtain the boundedness of $M$ on the Köthe dual space $H^{q^{\prime}, n(1-q / p)}(d x, \sigma)$ under the certain conditions:

Theorem 5.10. Let $1<q \leq p<\infty$ and $w \in H L M_{q}^{p}$ satisfy the weighted integral condition (2) for $p, q$ and $w$. Then $M$ is also bounded on $H^{q^{\prime}, n(1-q / p)}(d x, \sigma)$ with $\sigma(x)=w(x)^{-\frac{q^{\prime}}{q}}$ :

$$
\|M f\|_{H^{q^{\prime}, n(1-q / p)}(d x, \sigma)} \leq C\|f\|_{H^{q^{\prime}, n(1-q / p)}(d x, \sigma)} \quad\left(f \in H^{q^{\prime}, n(1-q / p)}(d x, \sigma)\right) .
$$

Many mathematicians have considered the equivalent BMO norms and we list the following:

(1) (Izuki 14] Let $p(\cdot): \mathbb{R}^{n} \rightarrow(0, \infty)$ be a measurable function. The variable Lebesgue norm $\|f\|_{L^{p(\cdot)\left(\mathbb{R}^{n}\right)}}$ is defined by

$$
\|f\|_{L^{p(\cdot)\left(\mathbb{R}^{n}\right)}}:=\inf \left\{\lambda>0: \int_{\mathbb{R}^{n}}\left|\frac{f(x)}{\lambda}\right|^{p(x)} d x \leq 1\right\} .
$$

Kováčik and Rákosník 21] have established that the generalized Lebesgue space $L^{p(\cdot)}\left(\mathbb{R}^{n}\right)$ with variable exponent $p(\cdot)$ is a Banach function space and its associate space is $L^{p^{\prime}(\cdot)}\left(\mathbb{R}^{n}\right)$ with norm equivalence, where $p^{\prime}(\cdot)$ denotes the conjugate exponent given by $\frac{1}{p(\cdot)}+\frac{1}{p^{\prime}(\cdot)}=1$.

The generalized Lebesgue space $L^{p(\cdot)}\left(\mathbb{R}^{n}\right)$ collects all measurable functions $f$ such that the norm $\|f\|_{L^{p(\cdot)}\left(\mathbb{R}^{n}\right)}$ is finite. 
For a measurable function $p(\cdot): \mathbb{R}^{n} \rightarrow[1, \infty)$, we generalize the norm (19) to

$$
\|b\|_{\mathrm{BMO}_{L^{p(\cdot)}}}:=\sup _{Q \in \mathcal{Q}} \frac{1}{\left\|\chi_{Q}\right\|_{L^{p(\cdot)}\left(\mathbb{R}^{n}\right)}}\left\|\left(b-b_{Q}\right) \chi_{Q}\right\|_{L^{p(\cdot)}\left(\mathbb{R}^{n}\right)} .
$$

If $p(\cdot)$ satisfies $\log p(\cdot) \in L^{\infty}$ and the Hardy-Littlewood maximal operator $M$ is bounded on $L^{p(\cdot)}\left(\mathbb{R}^{n}\right)$, then the generalized BMO norm $\|b\|_{\mathrm{BMO}_{L^{p(\cdot)}}}$ is equivalent to the classical one $\|b\|_{\text {BMO. }}$

(2) (Izuki and Sawano [16]) If a bounded measurable function $p(\cdot): \mathbb{R}^{n} \rightarrow[1, \infty)$ satisfies $1 \leq \inf p(x)$ and the log-Hölder conditions:

$$
\begin{gathered}
|p(x)-p(y)| \leq \frac{C}{-\log (|x-y|)} \quad \text { for } \quad x, y \in \mathbb{R}^{n},|x-y| \leq 1 / 2, \\
\left|p(x)-p_{\infty}\right| \leq \frac{C}{\log (e+|x|)} \quad \text { for } \quad x \in \mathbb{R}^{n},
\end{gathered}
$$

for some constants $C$ and $p_{\infty}$ independent of $x, y$, then $\|b\|_{\mathrm{BMO}_{L^{p(\cdot)}}}$ and $\|b\|_{\mathrm{BMO}}$ are equivalent.

(3) (Izuki, Sawano and Tsutsui [17]) If a measurable function $p(\cdot): \mathbb{R}^{n} \rightarrow[1, \infty)$ is bounded and $M$ is of weak type $(p(\cdot), p(\cdot))$, namely, there exists a constant $C>0$ such that for all $f \in L^{p(\cdot)}\left(\mathbb{R}^{n}\right)$ and all $\lambda>0$,

$$
\left\|\chi_{\{M f>\lambda\}}\right\|_{L^{p(\cdot)}\left(\mathbb{R}^{n}\right)} \leq C \lambda^{-1}\|f\|_{L^{p(\cdot)}\left(\mathbb{R}^{n}\right)},
$$

then $\|b\|_{\mathrm{BMO}_{L}(\cdot)}$ and $\|b\|_{\mathrm{BMO}}$ are equivalent.

(4) (Ho 12] Ho characterized the BMO norm in the context of general function space including Lebesgue spaces. Given a Banach function space $X$ equipped with a norm $\|\cdot\|_{X}$, we define the generalized BMO norm

$$
\|b\|_{\mathrm{BMO}_{X}}:=\sup _{Q \in \mathcal{Q}} \frac{1}{\left\|\chi_{Q}\right\|_{X}}\left\|\left(b-b_{Q}\right) \chi_{Q}\right\|_{X} .
$$

If $M$ is bounded on the associate space $X^{\prime}$, then $\|b\|_{\mathrm{BMO}_{X}}$ and $\|b\|_{\mathrm{BMO}}$ are equivalent. We remark that Ho's result [12] has covered the authors' one [14, 16]. The statements in [14, 16] are deeply depending on Diening's work [7] on variable exponent analysis. On the other hand, Ho's proof is self-contained and obtained as a by-product of the new results about atomic decomposition introduced in [12.

We can generalize Ho's result [12] to the ball Banach function spaces as before. Hence, by combining Theorem 5.10, we obtain the following:

Corollary 5.11. Let $1<q \leq p<\infty$ and $w \in H L M_{q}^{p}$ satisfy the weighted integral condition (2) for $p, q$ and $w$. Define a generalized BMO norm with respect to the weighted Morrey space of Samko's type

$$
\|b\|_{\mathrm{BMO}_{\mathcal{M}_{q}^{p}(d x, w)}}:=\sup _{Q \in \mathcal{Q}} \frac{1}{\Phi_{p, q, w}(Q)}\left\|\left(b-b_{Q}\right) \chi_{Q}\right\|_{\mathcal{M}_{q}^{p}(d x, w)} .
$$

Then the norm equivalence

$$
\|b\|_{\mathrm{BMO}_{\mathcal{M}_{q}^{p}(d x, w)}} \sim\|b\|_{\mathrm{BMO}}
$$

holds for all $b \in \mathrm{BMO}$.

\section{Appendix}

For completeness, we shall prove some lemmas used without the proof in this paper, nevertheless some of them are fundamental. 
Proof of (33). We may assume that $f$ is real-valued. Since

$$
\tan ^{-1}\left(m_{f}(Q)\right)=m_{\tan ^{-1} f}(Q),
$$

we may assume that $f$ is bounded. Also, the matters are local, we can assume that $f$ has compact support. Consequently, we can assume $f \in L^{1}\left(\mathbb{R}^{n}\right)$. In this case, we have the maximal estimate:

$$
\sup _{Q \in \mathcal{Q}} \chi_{Q}(x)\left|m_{f}(Q)\right| \leq 3 M f(x) .
$$

Since (33) is trivial for continous functions, we can go to the routine density argument.

Proof of Lemma 2.8, Our aim is to find $r>1$ such that

$$
\left\|M^{(r)} f\right\|_{\mathcal{M}_{q}^{p}(d x, w)} \leq C\|f\|_{\mathcal{M}_{q}^{p}(d x, w)} \quad\left(f \in \mathcal{M}_{q}^{p}(d x, w)\right),
$$

by assuming that $M$ is bounded on $\mathcal{M}_{q}^{p}(d x, w)$. We set for $f \geq 0$,

$$
R f(x):=\sum_{k=0}^{\infty} \frac{M^{k} f(x)}{\left(2 \alpha_{p, q, w}\right)^{k}}
$$

where $\alpha_{p, q, w}:=\|M\|_{\mathcal{M}_{q}^{p}(d x, w) \rightarrow \mathcal{M}_{q}^{p}(d x, w)}$. We observe that $R f \in A_{1}$. More precisely, we notice that

$$
[R f]_{A_{1}} \sim \operatorname{esssup}_{x \in \mathbb{R}^{n}} \frac{2 \alpha_{p, q, w} M\left[\sum_{k=0}^{\infty}\left(2 \alpha_{p, q, w}\right)^{-(k+1)} M^{k} f\right](x)}{f(x)+\sum_{k=0}^{\infty}\left(2 \alpha_{p, q, w}\right)^{-(k+1)} M^{k+1} f(x)} \leq 2 \alpha_{p, q, w} .
$$

Then we see that by [27, Lemma 2.1], there exists $r=r\left(\alpha_{p, q, w}\right)>1$ such that

$$
M^{(r)}[R f](x) \leq 2[R f]_{A_{1}} R f(x) \leq 4 \alpha_{p, q, w} R f(x) .
$$

Since it is clear that $f(x) \leq R f(x)$, it follows that $M^{(r)} f(x) \leq 4 \alpha_{p, q, w} R f(x)$. Hence, we see that

$$
\begin{aligned}
\left\|M^{(r)} f\right\|_{\mathcal{M}_{q}^{p}(d x, w)} & \leq 4 \alpha_{p, q, w}\|R f\|_{\mathcal{M}_{q}^{p}(d x, w)} \\
& \leq 4 \alpha_{p, q, w} \sum_{k=0}^{\infty} \frac{\left\|M^{k} f\right\|_{\mathcal{M}_{q}^{p}(d x, w)}}{\left(2 \alpha_{p, q, w}\right)^{k}} \\
& \leq 4 \alpha_{p, q, w} \sum_{k=0}^{\infty} 2^{-k}\|f\|_{\mathcal{M}_{q}^{p}(d x, w)} .
\end{aligned}
$$

To show Proposition 4.1, the following are crucial:

Lemma 6.1. Let $w \in A_{\infty}, Q_{0} \in \mathcal{Q}, f: Q_{0} \rightarrow \mathbb{R}$ and $\lambda_{w}, \lambda_{w}^{\prime}$ as before. Then there exists $\left\{Q_{j}^{1}\right\}_{j \in J_{1}} \subset \mathcal{D}\left(Q_{0}\right)$ such that

(1) $\left\{Q_{j}^{1}\right\}_{j \in J_{1}}$ are pairwise disjointed;

(2) $\sum_{j \in J_{1}}\left|Q_{j}^{1}\right| \leq \lambda_{w}^{\prime}\left|Q_{0}\right|$

(3) (decomposition formula)

$$
f-m_{f}\left(Q_{0}\right)=g_{1}+\sum_{j \in J_{1}} \alpha_{j, 1} \chi_{Q_{j}^{1}}+\sum_{j \in J_{1}}\left(f-m_{f}\left(Q_{j}^{1}\right)\right) \chi_{Q_{j}^{1}},
$$

where $g_{1}$ and $\alpha_{j, 1}$ satisfy

$$
\left|g_{1}\right| \leq 2 M_{\lambda_{w} ; Q_{0}}^{\sharp, d} f \cdot \chi_{Q_{0} \backslash \Omega_{1}} \quad\left(\Omega_{1}:=\bigcup_{j \in J_{1}} Q_{j}^{1}\right), \quad\left|\alpha_{j, 1}\right| \leq 2 \omega_{\lambda_{w}}\left(f ; Q_{0}\right) .
$$


Proof of Lemma 6.1. We put $f_{1}:=f-m_{f}\left(Q_{0}\right)$ and

$$
E_{1}:=\left\{x \in Q_{0}:\left|f_{1}(x)\right|>\left(f \cdot \chi_{Q_{0}}\right)^{*}\left(\lambda_{w}\left|Q_{0}\right|\right)\right\} .
$$

Moreover, we define

$$
\begin{aligned}
& \mathfrak{m}_{Q_{0}} f(x):=\sup _{\substack{Q \in \mathcal{D}\left(Q_{0}\right): \\
Q \ni x}} \max _{\substack{R \in \mathcal{D}\left(Q_{0}\right): \\
R^{(1)}=Q}}\left|m_{f_{1}}(R)\right|, \\
& \Omega_{1}:=\left\{x \in Q_{0}: \mathfrak{m}_{Q_{0}} f(x)>\left(f_{1} \cdot \chi_{Q_{0}}\right)^{*}\left(\lambda_{w}\left|Q_{0}\right|\right)\right\},
\end{aligned}
$$

where $R^{(1)}$ denotes the dyadic parent of $R$. Note that $\lim _{Q \ni x:|Q| \rightarrow 0} m_{f_{1}}(Q)=f_{1}(x)$ for a.e. $x \in \mathbb{R}^{n}$; see [6, Lemma 2.2] or [13, Proposition 3.1] as well. Hence, we see that $\left|f_{1}(x)\right| \leq \mathfrak{m}_{Q_{0}} f(x)$ which implies $E_{1} \subset \Omega_{1}$ almost everywhere. If $\left|E_{1}\right|=0$, then we have

$$
\left|f_{1}(x)\right| \leq 2 \omega_{\lambda_{w}}\left(f ; Q_{0}\right) \leq 2 M_{\lambda_{w} ; Q_{0}}^{\sharp, d} f(x),
$$

for a.e. $x \in Q_{0}$ by (34), and hence we complete the proof by letting $J_{1}=\emptyset$. Let us assume $\left|E_{1}\right|>0$. We also have $\left|\Omega_{1}\right|>0$. For each $x \in \Omega_{1}$, there exists $Q(x) \in \mathcal{D}\left(Q_{0}\right)$ such that $Q(x) \ni x$ and that

$$
\max _{\substack{R \in \mathcal{D}\left(Q_{0}\right): \\ R^{(1)}=Q(x)}}\left|m_{f_{1}}(R)\right|>\left(f_{1} \cdot \chi_{Q_{0}}\right)^{*}\left(\lambda_{w}\left|Q_{0}\right|\right) .
$$

The family $\mathcal{A}:=\{Q(x)\}_{x \in \Omega_{1}}$ covers $\Omega_{1}$. By considering the maximal dyadic cubes, we obtain $\left\{Q_{j}^{1}\right\}_{j \in J_{1}}$. It can not happen that $Q(x)=Q_{0}$, that is, $Q_{0} \notin\left\{Q_{j}^{1}\right\}_{j \in J_{1}}$, since $\lambda_{w}<\frac{1}{2}$. We first observe that for any $j \in J_{1}$,

$$
\left|m_{f_{1}}\left(Q_{j}^{1}\right)\right| \leq\left(f \cdot \chi_{Q_{0}}\right)^{*}\left(\lambda_{w}\left|Q_{0}\right|\right) .
$$

In fact, once we assume $\left|m_{f_{1}}\left(Q_{j}^{1}\right)\right|>\left(f \cdot \chi_{Q_{0}}\right)^{*}\left(\lambda_{w}\left|Q_{0}\right|\right)$, then for the dyadic parent of $Q_{j}^{1}$ denoted by $\left(Q_{j}^{1}\right)^{(1)}$, we have that

$$
\max _{\substack{R \in \mathcal{D}\left(Q_{0}\right): \\ R^{(1)}=\left(Q_{j}^{1}\right)^{(1)}}}\left|m_{f_{1}}(R)\right| \geq\left|m_{f_{1}}\left(Q_{j}^{1}\right)\right|>\left(f \cdot \chi_{Q_{0}}\right)^{*}\left(\lambda_{w}\left|Q_{0}\right|\right),
$$

which implies $\left(Q_{j}^{1}\right)^{(1)} \in \mathcal{A}$. However, this contradict the maximality of $Q_{j}^{1}$.

Now, with the disjointness of $\left\{Q_{j}^{1}\right\}_{j \in J_{1}}$ in mind, we decompose

$$
f-m_{f}\left(Q_{0}\right)=f_{1}=f_{1} \cdot \chi_{Q_{0} \backslash \Omega_{1}}+\sum_{j \in J_{1}} m_{f_{1}}\left(Q_{j}^{1}\right) \chi_{Q_{j}^{1}}+\sum_{j \in J_{1}}\left(f_{1}-m_{f_{1}}\left(Q_{j}^{1}\right)\right) \chi_{Q_{j}^{1}} .
$$

For the third term, we notice that

$$
f_{1}-m_{f_{1}}\left(Q_{j}^{1}\right)=f-m_{f}\left(Q_{0}\right)-\left(m_{f}\left(Q_{j}^{1}\right)-m_{f}\left(Q_{0}\right)\right)=f-m_{f}\left(Q_{j}^{1}\right) .
$$

Hence, by putting $g_{1}:=f_{1} \cdot \chi_{Q_{0} \backslash \Omega_{1}}$ and $\alpha_{j, 1}:=m_{f_{1}}\left(Q_{j}^{1}\right)$, we have

$$
f-m_{f}\left(Q_{0}\right)=g_{1}+\sum_{j \in J_{1}} \alpha_{j, 1} \chi_{Q_{j}^{1}}+\sum_{j \in J_{1}}\left(f-m_{f}\left(Q_{j}^{1}\right)\right) \chi_{Q_{j}^{1}} .
$$

For the second term, by recalling (63), it follows that

$$
\left|\alpha_{j, 1}\right| \leq\left(f_{1} \cdot \chi_{Q_{0}}\right)^{*}\left(\lambda_{w}\left|Q_{0}\right|\right) \leq 2 \omega_{\lambda_{w}}\left(f ; Q_{0}\right) .
$$

For the first term, if we notice that for $x \in Q_{0} \backslash \Omega_{1},\left|f_{1}(x)\right| \leq \mathfrak{m}_{Q_{0}} f(x) \leq\left(f_{1} \cdot \chi_{Q_{0}}\right)^{*}\left(\lambda_{w}\left|Q_{0}\right|\right)$, then we have

$$
\left|g_{1}(x)\right| \leq\left(f_{1} \cdot \chi_{Q_{0}}\right)^{*}\left(\lambda_{w}\left|Q_{0}\right|\right) \leq 2 M_{\lambda_{w} ; Q_{0}}^{\sharp, d} f(x) .
$$


Thus, it remains to show $\sum_{j \in J_{1}}\left|Q_{j}^{1}\right| \leq \lambda_{w}^{\prime}\left|Q_{0}\right|$. By the choice of $Q_{j}^{1}$ : (62), we notice that

$$
\begin{aligned}
\left(f_{1} \cdot \chi_{Q_{0}}\right)^{*}\left(\lambda_{w}\left|Q_{0}\right|\right)<\max _{\substack{R \in \mathcal{D}\left(Q_{0}\right): \\
R^{(1)}=Q_{j}^{1}}}\left|m_{f_{1}}(R)\right| \\
\leq \max _{\substack{R \in \mathcal{D}\left(Q_{0}\right): \\
R^{(1)}=Q_{j}^{1}}}\left(f_{1} \cdot \chi_{R}\right)^{*}\left(2^{-2}|R|\right) \\
\leq\left(f_{1} \cdot \chi_{Q_{j}^{1}}\right)^{*}\left(2^{-n-2}\left|Q_{j}^{1}\right|\right),
\end{aligned}
$$

which implies that

$$
\left|\left\{x \in Q_{j}^{1}:\left|f_{1}(x)\right|>\left(f_{1} \cdot \chi_{Q_{0}}\right)^{*}\left(\lambda_{w}\left|Q_{0}\right|\right)\right\}\right| \geq 2^{-n-2}\left|Q_{j}^{1}\right| .
$$

Thus, by summing up for $j \in J_{1}$, we see that

$$
\sum_{j \in J_{1}}\left|Q_{j}^{1}\right| \leq 2^{n+2}\left|\left\{x \in Q_{0}:\left|f_{1}(x)\right|>\left(f_{1} \cdot \chi_{Q_{0}}\right)^{*}\left(\lambda_{w}\left|Q_{0}\right|\right)\right\}\right| \leq 2^{n+2} \lambda_{w}\left|Q_{0}\right|=\lambda_{w}^{\prime}\left|Q_{0}\right| .
$$

Using Lemma 6.1, let us show Proposition 4.1]

Proof of Proposition 4.1. We employ the same notations as in Lemma 6.1. We put $\mathfrak{A}_{0}\left(Q_{0}\right):=$ $\left\{Q_{0}\right\}$ and $\mathfrak{A}_{1}\left(Q_{0}\right):=\left\{Q_{j}^{1}\right\}_{j \in J_{1}}$. In view of Lemma 6.1, we denote the map $\mathcal{Q} \ni Q \mapsto$ $\left\{Q_{j}(Q)\right\}_{j \in J(Q)}$ by $\mathfrak{R}$, where $\left\{Q_{j}(Q)\right\}_{j \in J(Q)}$ is a family of cubes generated by Lemma 6.1 , Note that $\mathfrak{R}\left(Q_{0}\right)=\mathfrak{A}_{1}\left(Q_{0}\right)$. Then we set

$$
\mathfrak{A}_{2}\left(Q_{0}\right):=\bigcup_{Q \in \mathfrak{A}_{1}\left(Q_{0}\right)} \mathfrak{R}(Q)=\bigcup_{j \in J_{1}} \mathfrak{R}\left(Q_{j}^{1}\right), \quad \mathfrak{A}_{k+1}\left(Q_{0}\right):=\bigcup_{Q \in \mathfrak{A}_{k}\left(Q_{0}\right)} \mathfrak{R}(Q)
$$

for all $k \in \mathbb{N}$. Then we can rephrase (61) as follows:

$$
f-m_{f}\left(Q_{0}\right)=g_{1}+\sum_{Q \in \mathfrak{A}_{1}\left(Q_{0}\right)} \alpha_{Q} \chi_{Q}+\sum_{Q \in \mathfrak{A}_{1}\left(Q_{0}\right)}\left(f-m_{f}(Q)\right) \chi_{Q} .
$$

Moreover, from Lemma 6.1 for each $f-m_{f}(Q)$ in the third term, we deduce that

$$
\begin{aligned}
f-m_{f}\left(Q_{0}\right)= & g_{1}+\sum_{Q \in \mathfrak{A}_{1}\left(Q_{0}\right)} \alpha_{Q} \chi_{Q} \\
& +\sum_{Q \in \mathfrak{A}_{1}\left(Q_{0}\right)}\left(g_{Q}+\sum_{R \in \mathfrak{R}(Q)} \alpha_{R} \chi_{R}+\sum_{R \in \mathfrak{R}(Q)}\left(f-m_{f}(R)\right) \chi_{R}\right),
\end{aligned}
$$

where $g_{Q}, \alpha_{R}$ and $R \in \mathfrak{R}(Q)$ satisfy the conditions in Lemma 6.1. Now, by observing that $\sum_{Q \in \mathfrak{A}_{1}\left(Q_{0}\right)} \sum_{R \in \mathfrak{R}(Q)}=\sum_{Q \in \mathfrak{A}_{2}\left(Q_{0}\right)}$ and putting $g_{2}:=g_{1}+\sum_{Q \in \mathfrak{A}_{1}\left(Q_{0}\right)} g_{Q}$, we have that

$$
f-m_{f}\left(Q_{0}\right)=g_{2}+\sum_{k=1}^{2} \sum_{Q \in \mathfrak{A}_{k}\left(Q_{0}\right)} \alpha_{Q} \chi_{Q}+\sum_{Q \in \mathfrak{A}_{2}\left(Q_{0}\right)}\left(f-m_{f}(Q)\right) \chi_{Q} .
$$

We claim the following four properties:

$$
\begin{gathered}
\left|g_{2}\right| \leq 2 M_{\lambda_{w} ; Q_{0}}^{\sharp, d} f \cdot \chi_{Q_{0} \backslash \cup_{Q \in \mathfrak{A}_{2}\left(Q_{0}\right)} Q}, \\
\left|\sum_{k=1}^{2} \sum_{Q \in \mathfrak{A}_{k}\left(Q_{0}\right)} \alpha_{Q} \chi_{Q}\right| \leq 2 \sum_{k=1}^{2} \sum_{Q \in \mathfrak{A}_{k-1}\left(Q_{0}\right)} \omega_{\lambda_{w}}(f ; Q) \chi_{Q}, \\
\sum_{Q \in \mathfrak{A}_{2}\left(Q_{0}\right)}|Q| \leq \lambda_{w}^{\prime 2}\left|Q_{0}\right|,
\end{gathered}
$$


and that $\left\{Q_{j}^{k}\right\}_{k=0,1,2, j \in J_{k}}:=\mathfrak{A}_{0}\left(Q_{0}\right) \cup \mathfrak{A}_{1}\left(Q_{0}\right) \cup \mathfrak{A}_{2}\left(Q_{0}\right)$ is a $w$-sparse family. For the first property (64), we have only to use the pointwise estimate of $g_{1}$ and $g_{Q}$ to get

$$
\begin{aligned}
\left|g_{2}\right| & \leq\left|g_{1}\right|+\sum_{Q \in \mathfrak{A}_{1}\left(Q_{0}\right)}\left|g_{Q}\right| \\
& \leq 2 M_{\lambda_{w} ; Q_{0}}^{\sharp, d} f \cdot \chi_{Q_{0} \backslash \bigcup_{Q \in \mathfrak{H}_{1}\left(Q_{0}\right)} Q}+\sum_{Q \in \mathfrak{A}_{1}\left(Q_{0}\right)} 2 M_{\lambda_{w} ; Q}^{\sharp, d} f \cdot \chi_{Q \backslash \bigcup_{R \in \mathfrak{R}(Q)} R} \\
& \leq 2 M_{\lambda_{w} ; Q_{0}}^{\sharp, d} f \cdot\left(\chi_{Q_{0} \backslash \bigcup_{Q \in \mathfrak{A}_{1}\left(Q_{0}\right)} Q}+\sum_{Q \in \mathfrak{A}_{1}\left(Q_{0}\right)} \chi_{\left.Q \backslash \bigcup_{R \in \mathfrak{R}(Q)} R\right)}\right) \\
& =2 M_{\lambda_{w} ; Q_{0}}^{\sharp, d} f \cdot \chi_{Q_{0} \backslash \bigcup_{Q \in \mathfrak{A}_{2}\left(Q_{0}\right)} Q} .
\end{aligned}
$$

For the property (65), by observing $\sum_{R \in \mathfrak{R}(Q)} \chi_{R} \leq \chi_{Q}$, we obtain

$$
\begin{aligned}
\left|\sum_{k=1}^{2} \sum_{Q \in \mathfrak{A}_{k}\left(Q_{0}\right)} \alpha_{Q} \chi_{Q}\right| & \leq 2 \omega_{\lambda_{w}}\left(f ; Q_{0}\right) \sum_{Q \in \mathfrak{A}_{1}\left(Q_{0}\right)} \chi_{Q}+\sum_{Q \in \mathfrak{A}_{1}\left(Q_{0}\right)} 2 \omega_{\lambda_{w}}(f ; Q) \sum_{R \in \mathfrak{R}(Q)} \chi_{R} \\
& \leq 2 \omega_{\lambda_{w}}\left(f ; Q_{0}\right) \chi_{Q_{0}}+\sum_{Q \in \mathfrak{A}_{1}\left(Q_{0}\right)} 2 \omega_{\lambda_{w}}(f ; Q) \chi_{Q} \\
& =2 \sum_{k=1}^{2} \sum_{Q \in \mathfrak{A}_{k-1}\left(Q_{0}\right)} \omega_{\lambda_{w}}(f ; Q) \chi_{Q} .
\end{aligned}
$$

For the third property (66), we have

$$
\sum_{Q \in \mathfrak{A}_{2}\left(Q_{0}\right)}|Q|=\sum_{Q \in \mathfrak{A}_{1}\left(Q_{0}\right)} \sum_{R \in \mathfrak{R}(Q)}|R| \leq \sum_{Q \in \mathfrak{A}_{1}\left(Q_{0}\right)} \lambda_{w}^{\prime}|Q| \leq \lambda_{w}^{\prime 2}\left|Q_{0}\right| .
$$

Finally, we show that $\left\{Q_{j}^{k}\right\}_{k=0,1,2, j \in J_{k}}$ is a $w$-sparse family. To this end, we have only to show that for $k=0,1$ and any $Q \in \mathfrak{A}_{k}\left(Q_{0}\right),\left|Q \cap \Omega_{k+1}\right| \leq \lambda_{w}^{\prime}|Q|$ holds, where $\Omega_{k}:=\bigcup_{Q \in \mathfrak{A}_{k}\left(Q_{0}\right)} Q$. Other properties of the $w$-sparse family is clear. When $k=0$, since $\mathfrak{A}_{0}\left(Q_{0}\right)=\left\{Q_{0}\right\}$, we see that

$$
\left|Q_{0} \cap \Omega_{1}\right|=\left|\bigcup_{Q \in \mathfrak{A}_{1}\left(Q_{0}\right)} Q\right|=\sum_{Q \in \mathfrak{A}_{1}\left(Q_{0}\right)}|Q| \leq \lambda_{w}^{\prime}\left|Q_{0}\right| .
$$

When $k=1$, for any $\widetilde{Q} \in \mathfrak{A}_{1}\left(Q_{0}\right)$, we have that

$$
\left|\widetilde{Q} \cap \Omega_{2}\right|=\left|\widetilde{Q} \cap\left[\bigcup_{Q \in \mathfrak{A}_{1}\left(Q_{0}\right)}\left(\bigcup_{R \in \Re(Q)} R\right)\right]\right|=\left|\bigcup_{R \in \Re(\widetilde{Q})} R\right| \leq \lambda_{w}^{\prime}|\widetilde{Q}| .
$$

As a result, we see that the four properties.

By repeating this procedure, we can decompose

$$
f-m_{f}\left(Q_{0}\right)=g_{K}+\sum_{k=1}^{K} \sum_{Q \in \mathfrak{A}_{k}\left(Q_{0}\right)} \alpha_{Q} \chi_{Q}+\sum_{Q \in \mathfrak{A}_{K}\left(Q_{0}\right)}\left(f-m_{f}(Q)\right) \chi_{Q},
$$

for any $K \in \mathbb{N}$. Here, $\left\{g_{k}\right\}_{k=1, \ldots, K},\left\{\alpha_{Q}\right\}_{Q \in \mathfrak{A}_{k}\left(Q_{0}\right), k=1, \ldots, K}$ and $\left\{Q_{j}^{k}\right\}_{k=0, \ldots, K, j \in J_{k}}:=\bigcup_{k=0}^{K} \mathfrak{A}_{k}\left(Q_{0}\right)$ satisfy the analogy of the four properties claimed in the case of $K=2$ as above. In particular, we have

$$
\sum_{Q \in \mathfrak{A}_{K}\left(Q_{0}\right)}|Q| \leq \lambda_{w}^{\prime K}\left|Q_{0}\right| \rightarrow 0 \quad(K \rightarrow \infty)
$$


which yields that for a.e. $x \in Q_{0}$,

$$
\sum_{Q \in \mathfrak{A}_{K}\left(Q_{0}\right)}\left(f-m_{f}(Q)\right) \chi_{Q} \rightarrow 0 \quad(K \rightarrow \infty) .
$$

Hence, by letting $\mathfrak{A}_{k}\left(Q_{0}\right):=\left\{Q_{j}^{k}\right\}_{j \in J_{k}}$, we conclude that

$$
\begin{aligned}
\left|f-m_{f}\left(Q_{0}\right)\right| & \leq 2 M_{\lambda_{w} ; Q_{0}}^{\sharp, d} f+2 \sum_{k=1}^{\infty} \sum_{Q \in \mathfrak{A}_{k-1}\left(Q_{0}\right)} \omega_{\lambda_{w}}(f ; Q) \chi_{Q} \\
& =2 M_{\lambda_{w} ; Q_{0}}^{\sharp, d} f+2 \sum_{k=0}^{\infty} \sum_{j \in J_{k}} \omega_{\lambda_{w}}\left(f ; Q_{j}^{k}\right) \chi_{Q_{j}^{k}} .
\end{aligned}
$$

By the construction, $\left\{Q_{j}^{k}\right\}_{k \in \mathbb{N}_{0}, j \in J_{k}}=\bigcup_{k \in \mathbb{N}_{0}} \mathfrak{A}_{k}\left(Q_{0}\right)$ is a $w$-sparse family.

Next, we prove the estimate (51).

Proof of (51). We put $\varepsilon:=2^{-(n+3)}[w]_{A_{\infty}}^{-1}$ and take $L_{w} \in \mathbb{N}$ so that $L_{w}>n^{-1}\left(1+\frac{1}{\varepsilon}\right)$. We also take $\alpha_{w}:=2^{n L_{w} \cdot \frac{\varepsilon}{1+\varepsilon}-1}>1$. It suffices to show that

$$
w\left(Q_{0}^{\left(i+L_{w}\right)}\right) \geq \alpha_{w} w\left(Q_{0}^{(i)}\right), \quad\left(i \in \mathbb{N}_{0}, Q_{0} \in \mathcal{D}\right) .
$$

We employ the reverse Hölder inequality to obtain that

$$
\begin{aligned}
w\left(Q_{0}^{(i)}\right) & \leq\left|Q_{0}^{(i)}\right|^{\frac{\varepsilon}{1+\varepsilon}}\left(\int_{Q_{0}^{\left(i+L_{w}\right)}} w(x)^{1+\varepsilon} d x\right)^{\frac{1}{1+\varepsilon}} \\
& \leq\left|Q_{0}^{(i)}\right|^{\frac{\varepsilon}{1+\varepsilon}}\left|Q_{0}^{\left(i+L_{w}\right)}\right|^{\frac{1}{1+\varepsilon}} \frac{2}{\left|Q_{0}^{\left(i+L_{w}\right)}\right|} w\left(Q_{0}^{\left(i+L_{w}\right)}\right) \\
& =2^{1-n L_{w} \frac{\varepsilon}{1+\varepsilon}} w\left(Q_{0}^{\left(i+L_{w}\right)}\right),
\end{aligned}
$$

which implies (67).

Finally, we prove Lemma 5.3

Proof of Lemma 5.3. In this proof, we denote the weighted mean value by $b_{Q}^{(w)}$, namely, $b_{Q}^{(w)}:=$ $\frac{1}{w(Q)} \int_{Q} b(x) w(x) d x$. Since it is well known that

$$
\|b\|_{\mathrm{BMO}} \sim \sup _{Q \in \mathcal{Q}}\left(\frac{1}{w(Q)} \int_{Q}\left|b(x)-b_{Q}^{(w)}\right|^{q} w(x) d x\right)^{\frac{1}{q}}
$$

for all $0<q<\infty$ when $w$ is a $A_{\infty}$ weight; for example [28, we have that

$$
\left(\frac{1}{w(Q)} \int_{Q}\left|b(x)-b_{Q}\right|^{q} w(x) d x\right)^{\frac{1}{q}} \leq C\|b\|_{\mathrm{BMO}}+\left|b_{Q}-b_{Q}^{(w)}\right| .
$$

Moreover, with $w \in A_{\infty}$ in mind, we have that

$$
\begin{aligned}
\left|b_{Q}-b_{Q}^{(w)}\right| & \leq \frac{1}{w(Q)} \int_{Q}\left|b(y)-b_{Q}\right| w(y) d y \\
& =\frac{1}{w(Q)} \int_{0}^{\infty} w\left(\left\{y \in Q:\left|b(y)-b_{Q}\right|>t\right\}\right) d t \\
& \leq C \int_{0}^{\infty}\left(\frac{\left|\left\{y \in Q:\left|b(y)-b_{Q}\right|>t\right\}\right|}{|Q|}\right)^{\delta} d t
\end{aligned}
$$


for some $\delta \in(0,1)$. Now, by invoking the John-Nirenberg inequality, it follows that

$$
\left|b_{Q}-b_{Q}^{(w)}\right| \leq C \int_{0}^{\infty} \exp \left[-\frac{c t \delta}{\|b\|_{\mathrm{BMO}}}\right] d t \sim\|b\|_{\mathrm{BMO}}
$$

\section{REFERENCES}

[1] D. R. Adams, J. Xiao, Morrey spaces in harmonic analysis, Ark. Mat., 50 (2012), no. 2, 201-230.

[2] C. Bennett, R. Sharpley, Interpolation of Operators, Academic Press, 1988.

[3] V. I. Burenkov, V. S. Guliyev, A. Serbetci and T. V. Tararykova, Necessary and sufficient condition for the boundedness of genuine singular integral operators in local Morrey-Type spaces, Eurasian Math. J. 1, no. $1,32-53,2010$.

[4] R. R. Coifman and C. Fefferman, Weighted norm inequalities for maximal functions and singular integrals, Studia Math., 51 241-250, 1974.

[5] D. Cruz-Uribe, SFO, E. Hernández and J. M. Martell, Greedy bases in variable Lebesgue spaces, arXiv:1410.1819

[6] N. Fujii, A condition for a two-weight norm inequality for singular integral operators, Studia Math., 98 (3) (1991), 175-190.

[7] L. Diening, Maximal functions on Musielak-Orlicz spaces and generalized Lebesgue spaces, Bull. Sci. Math. 129 (2005), 657-700.

[8] J. Duoandikoetxea, Fourier Analysis, Graduate Studies in Math. 29, Amer. Math. Soc., Providence, RI, 2001.

[9] J. Garcia-Cuerva, J. L. Rubio de Francia, Weighted Norm Inequalities and Related Topics, North-Holland, Math. Stud., 116 (1985).

[10] L. Grafakos, Moderen Fourier Analysis, Third Edition, Springer, GTM250.

[11] D. I. Hakim, E. Nakai and Y. Sawano, Generalized fractional maximal operators and vector-valued inequalities on generalized Orlicz-Morrey spaces, Revista Matemática Complutense, (2015), 1-32.

[12] K.-P. Ho, Atomic decomposition of Hardy spaces and characterization of BMO via Banach function spaces, Anal. Math. 38 (2012), 173-185.

[13] T. Hytönen, Weighted norm inequalities, Lecture notes of a course at the University of Helsinki, Winter 2011 , http://wiki-app.it.helsinki.fi/download/attachments/64424417 /weighted.pdf.

[14] M. Izuki, Boundedness of commutators on Herz spaces with variable exponent, Rend. Circ. Mat. Palermo (2) 59 (2010), 199-213.

[15] M. Izuki, Remarks on Muckenhoupt weights with variable exponent, J. Anal. Appl. 11 (2013), 27-42.

[16] M. Izuki and Y. Sawano, Variable Lebesgue norm estimates for BMO functtions, Czechoslovak Math. J. 62 (2012), 717-727.

[17] M. Izuki, Y. Sawano and Y. Tsutsui, Variable Lebesgue norm estimates for BMO functtions. II, Anal. Math. 40 (2014), 215-230.

[18] B. Jawerth and A. Torchinsky, Local sharp maximal functions, J. Approx. Theory, 43 (1965), 231-270.

[19] F. John, Quasi-isometric mappings, Seminari 1962-1963 di Analisi, Algebra, Geometria e Topologia, Ist Nazarene Alta Matematica 2 (Edizioni Cremonese, Rome, 1965), 462-473.

[20] J. John and L. Nirenberg, On functions of bounded mean oscillation, Comm. Pure Appl. Math. 14 (1961), $415-426$.

[21] O. Kováčik and J. Rákosník, On spaces $L^{p(x)}$ and $W^{k, p(x)}$, Czech. Math. J. 41 (1991), 592-618.

[22] Y. Komori-Furuya, Local Good- $\lambda$ Estimate for the Sharp Maximal Function and Weighted Morrey Space, Journal of Function Spaces Volume 2015 (2015), Article ID 651825, 4 pages http://dx.doi.org/10.1155/2015/651825

[23] Y. Komori and S. Shirai, Weighted Morrey spaces and a singular integral operator, Math. Nachr. 282 (2009), no. 2, 219-231.

[24] A.K. Lerner, On weighted estimates of non-increasing rearrangements, East J. Approx. 4 (1998), 277-290.

[25] A.K. Lerner, Some remarks on the Fefferman-Stein inequality, J. Anal. Math. 112 (2010), 329-349.

[26] A.K. Lerner, On an estimate of Calderön-Zygmund operators by dyadic positive operators, J. Anal. Math. 121 (2013), 141-161.

[27] A.K. Lerner, S. Ombrosi, A boundedness criterion for general maximal operators, Publ. Mat., 54 (2010) no. $1,53-71$.

[28] B. Muckenhoupt, R. L. Wheeden, Weighted bounded mean oscillation and the Hilbert transform, Studia Math., 54 (1976), 221-237. 
[29] E. Nakai, Hardy-Littlewood maximal operator, singular integral operators and the Riesz potentials on generalized Morrey spaces, Math. Nachr. 166 (1994), 95-103.

[30] S. Nakamura, Generalized weighted Morrey spaces and classical operators, submitted.

[31] S. Nakamura, Embedding properties and trace theorem for weighted Morrey spaces, pre-print.

[32] S. Nakamura, T. Noi and Y. Sawano, Generalized Morrey spaces and trace operator, Sci. Math. China, online.

[33] N Samko, Weighted Hardy and singular operators in Morrey spaces, J. Math. Anal. Appl. 350 (2009), $56-72$.

[34] J. Orobitg, J. Verdera, Choquet integrals, Hausdorff content and the Hardy-Littlewood maximal operator, Bull. London Math. Soc., 30 (1998), no. 2, 145-150.

[35] C. Pérez, Endpoint estimates for commutators of singular integral operators, J. Funct. Anal., 128 (1995), 163-185.

[36] Y. Sawano and S. Shirai, Compact commutators on Morrey spaces with non-doubling measures, Georgian Math. J., 15 (2008), no. 2, 353-376.

[37] Y. Sawano and H. Tanaka, Sharp maximal inequalities and commutators on Morrey spaces with nondoubling measures, Taiwanese J. Math. 11 (2007), no. 4, 1091-1112.

[38] Y. Sawano and H. Tanaka, Predual spaces of Morrey spaces with non-doubling measures, Tokyo J. Math. 32 (2009), 471-486.

[39] Y. Sawano and H. Tanaka, Fatou property of predual Morrey spaces with non-doubling measures, Int. J. Appl. Math. 27, no. 3, 283-296 (2014).

[40] Y. Sawano, H. Tanaka, The Fatou Property of Block spaces, J. Math. Sci. Univ. Tokyo, 22, 663-683 (2015).

[41] H. Tanaka, Two-weight norm inequalities on Morrey spaces, Ann. Acad. Sci. Fenn. Math. 40 (2015), $773-791$.

[42] J.O. Strömberg, Bounded mean oscillation with Orlicz norms and duality of Hardy spaces, Indiana Univ. Math. J. 28 (1979), 511-544.

[43] C.T. Zorko, Morrey space, Proc. Amer. Math. Soc. 98 (1986), 586-592. 


\title{
THE SINGULAR INTEGRAL OPERATOR AND ITS COMMUTATOR ON WEIGHTED MORREY SPACES
}

\author{
SHOHEI NAKAMURA AND YOSHIHIRO SAWANO
}

\begin{abstract}
In this paper, we give necessary conditions and sufficient conditions respectively for the boundedness of the singular integral operator on the weighted Morrey spaces. We observe the phenomenon unique to the case of Morrey spaces; the $A_{q}$-theory by Muckenhoupt and Wheeden does not suffice. We also discuss the boundedness of the commutators. The difference between the maximal operator and the singular integral operators will be clarified. Further, the property of the sharp maximal operator is investigated.
\end{abstract}

\section{INTRODUCTION}

The weight theory on Lebesgue spaces have been developed by many mathematicians in terms of $A_{p}$ conditions and is also developing; see for example [4, 11, 20. As a remarkable recent progress, Hytönen [11] solved the " $A_{2}$ conjecture" which was an open problem on this field. Meanwhile, some mathematicians pay attention to weighted Morrey spaces and try to construct the weight theory on Morrey spaces; for example [15, 22, 24, 30. As is pointed out in [24, 30] implicitly, $A_{p}$ condition is not suitable on weighted Morrey spaces and hence, it is a natural problem to find the alternative condition. To tackel this problem, we discuss the boundedness of the singular integral operators on weighted Morrey spaces by assuming the boundedness of the maximal operator in this paper.

First of all, we fix our notation. We denote the set of the all Lebesgue measurable functions by $L^{0}\left(\mathbb{R}^{n}\right)$. By $\mathcal{Q}=\mathcal{Q}\left(\mathbb{R}^{n}\right)$, we mean the all cubes in $\mathbb{R}^{n}$ whose sides parallel to the coordinate axes. We denote the family of all dyadic cubes by $\mathcal{D}=\mathcal{D}\left(\mathbb{R}^{n}\right)$ and the family of all dyadic cubes with respect to $Q \in \mathcal{Q}$ by $\mathcal{D}(Q)$. A weight $w$ is a locally integrable function on $\mathbb{R}^{n}$ such that $w(x)>0$ for almost everywhere $x \in \mathbb{R}^{n}$. For weight $w$ and measurable set $E \subset \mathbb{R}^{n}$, we denote $w(E):=\int_{E} w(x) d x$. In addition, we denote a weighted measure by $d w$, that is, $d w(x)=w(x) d x$.

We define the weighted Morrey space $\mathcal{M}_{q}^{p}\left(w_{1}, w_{2}\right)$, where $0<q \leq p<\infty$ and $w_{1}, w_{2}$ are weights. The weighted Morrey space $\mathcal{M}_{q}^{p}\left(w_{1}, w_{2}\right)$ is the set of all functions $f \in L_{\text {loc }}^{q}\left(w_{2}\right)$ for which the quasi-norm

$$
\|f\|_{\mathcal{M}_{q}^{p}\left(w_{1}, w_{2}\right)}:=\sup _{Q \in \mathcal{Q}} w_{1}(Q)^{\frac{1}{p}-\frac{1}{q}}\left(\int_{Q}|f(x)|^{q} d w_{2}(x)\right)^{\frac{1}{q}}
$$

is finite. When $w_{1}=w_{2}=w, \mathcal{M}_{q}^{p}\left(w_{1}, w_{2}\right)$ corresponds to the weighted Morrey space $\mathcal{M}_{q}^{p}(w, w)$ introduced by Komori and Shirai in [15. Meanwhile, when $w_{1}=d x$ and $w_{2}=w, \mathcal{M}_{q}^{p}\left(w_{1}, w_{2}\right)$ corresponds to the weighted Morrey space $\mathcal{M}_{q}^{p}(d x, w)$ introduced by Samko in [24. Our main interest is the latter case: $\mathcal{M}_{q}^{p}(d x, w)$.

2010 Mathematics Subject Classification. Primary 42B20; Secondary 42B25, 46E30.

Key words and phrases. Weighted Morrey spaces, Singular integral operators, 4, commutator. 
In 22, we introduced the weight class $\mathcal{B}_{p, q}$ and the weighted integral condition in the context of the boundedness of the Hardy-Littlewood maximal operator on weighted Morrey spaces of Samko type.

Definition $1.1([22])$. Let $0<q \leq p<\infty$ and $w$ be a weight.

(1) One says that a weight $w$ is in the class $\mathcal{B}_{p, q}$ if there exists $C_{p, q}>0$ such that for any $Q_{0} \in \mathcal{Q}$,

$$
\sup _{Q \in \mathcal{Q}: Q \subset Q_{0}} \Phi_{p, q, w}(Q) \leq C_{p, q} \Phi_{p, q, w}\left(Q_{0}\right),
$$

or equivalently,

hold, where we defined

$$
\left\|\chi_{Q_{0}}\right\|_{\mathcal{M}_{q}^{p}(d x, w)} \sim \Phi_{p, q, w}\left(Q_{0}\right)
$$

$$
\Phi_{p, q, w}(Q):=|Q|^{\frac{1}{p}}\left(\frac{w(Q)}{|Q|}\right)^{\frac{1}{q}} \quad(Q \in \mathcal{Q}) .
$$

(2) The weighted integral condition for $p, q$ and $w$ holds, if there exists a constant $C>0$ such that

$$
\int_{1}^{\infty} \frac{1}{\Phi_{p, q, w}(s Q)} \frac{d s}{s} \leq \frac{C}{\Phi_{p, q, w}(Q)} \quad(Q \in \mathcal{Q})
$$

holds.

Note that the weighted integral condition (1.3) for $p, q$ and $w$ implies $w \in \mathcal{B}_{p, q}$; see 22 for details. Using these two notions, we aim to give necessary conditions and sufficiently conditions for the boundedness of the singular integral operators. To this end, we first recall the definition of the Hardy-Littlewood maximal operator and the Köthe dual space of Morrey spaces. By $M$, we mean the (unweighted) Hardy-Littlewood maximal operator:

$$
M f(x):=\sup _{Q \in \mathcal{D}} \frac{1}{|Q|} \int_{Q}|f(y)| d y \cdot \chi_{Q}(x) .
$$

To recall the Köthe dual spaces of Morrey spaces, we need some terminologies. Let $0<\alpha \leq n$ and $E \subset \mathbb{R}^{n}$ be an arbitrary set. Then the $\alpha$-dimensional Hausdorff content of $E$ is defined by

$$
H^{\alpha}(E):=\inf \left\{\sum_{j} \ell\left(Q_{j}\right)^{\alpha}:\left\{Q_{j}\right\}_{j} \subset \mathcal{Q}, \bigcup_{j} Q_{j} \supset E\right\} .
$$

For a non-negative function $\phi \geq 0$, the Choquet integral of $\phi$ with respect to the Hausdorff content $H^{\alpha}$ is defined by

$$
\int_{\mathbb{R}^{n}} \phi d H^{\alpha}:=\int_{0}^{\infty} H^{\alpha}\left(\left\{x \in \mathbb{R}^{n}: \phi(x)>t\right\}\right) d t .
$$

Definition 1.2 ([1]). Define $\mathfrak{B}_{\alpha}:=\left\{b \in A_{1}: \int_{\mathbb{R}^{n}} b d H^{\alpha} \leq 1\right\}$ for $0<\alpha<n$.

A typical example of $\mathfrak{B}_{\alpha}$ is

$$
b_{Q}:=\frac{\left(M \chi_{Q}\right)^{\alpha / n+\varepsilon}}{\ell(Q)^{\alpha}}
$$

where $Q \in \mathcal{Q}$ and $\varepsilon \in\left(0,1-\frac{\alpha}{n}\right)$ are arbitrary. Then for $1<q \leq p<\infty$, the space $H^{q^{\prime}, n(1-q / p)}(d x, d x)=H^{q^{\prime}, n(1-q / p)}$ is defined by the set of all measurable functions $f$ for which norm

$$
\|f\|_{H^{q^{\prime}, n(1-q / p)}}:=\inf _{b \in \mathfrak{B}_{n(1-q / p)}}\left(\int_{\mathbb{R}^{n}}|f(x)|^{q^{\prime}} b(x)^{-\frac{q^{\prime}}{q}} d x\right)^{\frac{1}{q^{\prime}}}
$$


is finite.

The following are proved in [1] or [30, (2.5)]: For $1<q \leq p<\infty$, we have

dual

$$
\left|\int_{\mathbb{R}^{n}} f(x) g(x) d x\right| \leq C\|f\|_{\mathcal{M}_{q}^{p}(d x, d x)}\|g\|_{H^{q^{\prime}, n(1-q / p)}},
$$

for all $f \in \mathcal{M}_{q}^{p}(d x, d x)$ and $g \in H^{q^{\prime}, n(1-q / p)}$ and that

dual2

$$
\|g\|_{H^{q^{\prime}, n(1-q / p)}} \sim \sup \left\{\|f \cdot g\|_{L^{1}}: f \in \mathcal{M}_{q}^{p}(d x, d x):\|f\|_{\mathcal{M}_{q}^{p}(d x, d x)} \leq 1\right\},
$$

for any measurable function $g$.

Now, we state our main results in this paper. The Riesz transform is defined by

$$
R_{i} f(x):=\lim _{\varepsilon \rightarrow 0} \int_{\left\{y \in \mathbb{R}^{n}:\left|x_{i}-y_{i}\right|>\varepsilon\right\}} \frac{x_{i}-y_{i}}{|x-y|^{n+1}} f(y) d y \quad\left(f \in L^{2}\left(\mathbb{R}^{n}\right)\right),
$$

for $i=1, \ldots, n$.

th-160626-1

item-160626-1

item-160626-2

$151221-1$

th-tanaka
Theorem 1.3. Let $1<q \leq p<\infty$ and $w$ be a weight.

(1) (Sufficient condition) Assume that the Hardy-Littlewood maximal operator $M$ is bounded on $\mathcal{M}_{q}^{p}(d x, w)$ and the weighted integral condition (1.3) for $p, q$ and $w$. Then for any $f \in \mathcal{M}_{q}^{p}(d x, w), R_{i} f$ is well defined and $R_{i}$ is bounded on $\mathcal{M}_{q}^{p}(d x, w)$ :

$$
\left\|R_{i} f\right\|_{\mathcal{M}_{q}^{p}(d x, w)} \leq C\|f\|_{\mathcal{M}_{q}^{p}(d x, w)} \quad\left(f \in \mathcal{M}_{q}^{p}(d x, w)\right) .
$$

(2) (Necessary condition) Conversely, assume $R_{i}$ is bounded on $\mathcal{M}_{q}^{p}(d x, w)$. Then we have

$$
\frac{1}{|Q|}\left\|\chi_{Q} w^{\frac{1}{q}}\right\|_{\mathcal{M}_{q}^{p}(d x, d x)}\left\|\chi_{Q} w^{-\frac{1}{q}}\right\|_{H^{q^{\prime}, n(1-q / p)}} \leq C \quad(Q \in \mathcal{Q}),
$$

and the weighted integral condition (1.3) for $p, q$ and $w$.

Remark 1.4. In Theorem 1.3, we have a "gap" between the sufficient condition (1) and the necessary condition (2), that is, a "gap" between the boundedness of $M$ and (1.7). To this problem, it was conjectured in 30] that the boundedness of $M$ on $\mathcal{M}_{q}^{p}(d x, w)$ is equivalent to (1.7). Hence, if the conjecture is true, then we obtain from Theorem 1.3 the characterization of the boundedness of $R_{i}$ on $\mathcal{M}_{q}^{p}(d x, w)$. That is, $R_{i}$ is bounded on $\mathcal{M}_{q}^{p}(d x, w)$ if and only if (1.7) and the weighted integral condition (1.3) hold.

As is well known, on the weighted Lebesgue spaces, the boundedness of $R_{i}$ or $M$ is equivalent to $A_{p}$ condition. However, as in Theorem [1.3. there exists an essential difference between the behavior of $R_{i}$ and $M$ on the weighted Morrey spaces coming from the weighted integral condition (1.3). Of course, we have possibility that the weighted integral condition (1.3) is not essential which means (1.3) always holds automatically. In fact, in the case of weighted Lebesgue space: $p=q$, the weighted integral condition (1.3) holds as long as $w$ is a doubling weight. To see that the weighted integral condition (1.3) is essential, we focus on the specific weight, namely, the power weight: $w(x)=w_{\alpha}(x):=|x|^{\alpha}$. On this specific situation, the problem of the boundedness of $M$ on $\mathcal{M}_{q}^{p}(d x, w)$ is completely solved in [30] as follows.

Theorem 1.5 (30]). Let $1<q<p<\infty$ and $\alpha>-n$. Then the following are equivalent:

(1) The boundedness of $M$ on $\mathcal{M}_{q}^{p}\left(d x, w_{\alpha}\right)$ :

$$
\|M f\|_{\mathcal{M}_{q}^{p}\left(d x, w_{\alpha}\right)} \leq C\|f\|_{\mathcal{M}_{q}^{p}\left(d x, w_{\alpha}\right)} \quad\left(f \in \mathcal{M}_{q}^{p}\left(d x, w_{\alpha}\right)\right) ;
$$

(2) The dual inequality (1.7) with $w=w_{\alpha}$ :

$$
\frac{1}{|Q|}\left\|\chi_{Q} w_{\alpha}^{\frac{1}{q}}\right\|_{\mathcal{M}_{q}^{p}(d x, d x)}\left\|\chi_{Q} w_{\alpha}^{-\frac{1}{q}}\right\|_{H^{q^{\prime}, n(1-q / p)}} \leq C \quad(Q \in \mathcal{Q}) ;
$$


(3) The range of $\alpha$ :

$$
-\frac{q}{p} n \leq \alpha<n\left(q-\frac{q}{p}\right) .
$$

Meanwhile, as is shown in 22, the weighted integral condition (1.3) for $p, q$ and $w_{\alpha}$ is equivalent to $\alpha>-\frac{q}{p} n$. Hence, we obtain the following:

cr-160113-2 Corollary 1.6. Let $1<q \leq p<\infty$ and $\alpha>-n$. The Riesz transform $R_{i}, i=1, \ldots, n$ is bounded on $\mathcal{M}_{q}^{p}\left(d x, w_{\alpha}\right)$ if and only if

$$
-\frac{q}{p} n<\alpha<n\left(q-\frac{q}{p}\right) .
$$

Comparing (1.8) and (1.9), we notice the essential difference appears at the end-point: $\alpha=$ $-\frac{q}{p}$.

We now compare our result to the known results. In 22, we proved the boundedness of $R_{i}$ on $\mathcal{M}_{q}^{p}(d x, w)$ by assuming $w \in A_{q}$ and the weighted integral condition (1.3). In particular, when the case of $w=w_{\alpha}$, the conditions: $w \in A_{q}$ and (1.3) are combined into:

$$
-\frac{q}{p} n<\alpha<n(q-1) .
$$

Hence, by comparing (1.9) and (1.10), we notice that Theorem 1.3 improves the result obtained in 22. When the case of $n=1$, in 24, Samko obtained the characterization of the boundedness of the Hilbert transform with power weights. Recall that the Hilbert transform $H$ is a singular integral operator defined by

$$
H f(x):=\lim _{\varepsilon \rightarrow 0} \int_{\{y \in \mathbb{R}:|x-y|>\varepsilon\}} \frac{f(y)}{x-y} d y \quad\left(f \in L^{2}(\mathbb{R})\right) .
$$

Note that the Hilbert transform $H$ is the special case of the Riezs transform $R_{i}$ with $n=1$. We can recapture the result by Samko.

NS Theorem 1.7 (24]). Let $1<q \leq p<\infty$ and $\alpha>-1$. The Hilbert transform $H$ is bounded on $\mathcal{M}_{q}^{p}\left(d x, w_{\alpha}\right)$, if and only if

$$
-\frac{q}{p}<\alpha<q-\frac{q}{p}
$$

We see that both Theorem 1.3 and Corollary 1.6 cover Theorem 1.7

We also discuss the boundedness of the commutators. The commutator of the Riesz transform $R_{i}$ with a locally integrable function $b$ is initially defined by

$$
\left[b, R_{i}\right] f:=b \cdot R_{i} f-R_{i}(b \cdot f),
$$

for $f \in L_{\mathrm{c}}^{\infty}$. A locally integrable function $b$ is said to be a BMO function, if

$$
\|b\|_{\mathrm{BMO}}:=\left\|b^{\sharp}\right\|_{L^{\infty}}<\infty,
$$

where $b^{\sharp}$ is the Fefferman-Stein-Stromberg sharp maximal function defined by (1.13). When $b$ is a BMO function, we can extend the commutator $\left[b, R_{i}\right]$ as a bounded linear operator on $L^{p}\left(\mathbb{R}^{n}\right)$ for all $1<p<\infty$; see [7. Theorem 3.5.6] for example. We also note that in the endpoint case: $p=1$, the commutators are more singular than the singular integral operators. We refer 25] for the result of the commutators in the endpoint case: $p=1$. Let $R_{i}, i=1, \ldots, n$ be the $i$-th Riesz transform. For the commutators, we obtain a similar result as follows. 
Theorem 1.8. Let $1<q \leq p<\infty, b \in \mathrm{BMO}$ and $i=1,2, \ldots, n$ be fixed. Assume that $M$ is bounded on $\mathcal{M}_{q}^{p}(d x, w)$ and the weighted integral condition (1.3) for $p, q$ and $w$. Then we can extend the commutator $\left[b, R_{i}\right]$ as a bounded linear operator on $\mathcal{M}_{q}^{p}(d x, w)$ :

$$
\left\|\left[b, R_{i}\right] f\right\|_{\mathcal{M}_{q}^{p}(d x, w)} \leq C\|f\|_{\mathcal{M}_{q}^{p}(d x, w)} \quad\left(f \in \mathcal{M}_{q}^{p}(d x, w)\right) .
$$

To prove Theorem 1.3, we will employ the sharp maximal operator arguments. To this end, we generalize the sharp maximal inequality on Lebesgue spaces to our Morrey spaces. Our results related to the sharp maximal inequality improve the well known sharp maximal inequality on Lebesgue spaces in some sense. We recall the local sharp maximal operator introduced in [13, 31].

Definition 1.9 ([10, 18]). Let $f \in L^{0}\left(\mathbb{R}^{n}\right)$ and $Q \in \mathcal{Q}$.

(1) The decreasing rearrangement of $f$ on $\mathbb{R}^{n}$ is defined by

$$
f^{*}(t):=\left\{\rho>0:\left|\left\{x \in \mathbb{R}^{n}:|f(x)|>\rho\right\}\right|<t\right\}, \quad(0<t<\infty) .
$$

(2) The local mean oscillation of $f$ on $Q$ is defined by

$$
\omega_{\lambda}(f ; Q):=\inf _{c \in \mathbb{C}}\left((f-c) \chi_{Q}\right)^{*}(\lambda|Q|), \quad\left(\lambda \in\left(0,2^{-1}\right)\right) .
$$

(3) Assume that the function $f$ is real-valued. The median of $f$ over $Q$ denoted by $m_{f}(Q)$ is a real number satisfying that

$$
\left|\left\{x \in Q: f(x)>m_{f}(Q)\right\}\right|, \quad\left|\left\{x \in Q: f(x)<m_{f}(Q)\right\}\right| \leq \frac{1}{2} .
$$

Note that the median $m_{f}(Q)$ is possibly non-unique.

For $\lambda \in\left(0,2^{-1}\right)$ and $Q_{0} \in \mathcal{Q}$, the dyadic local sharp maximal operator $M_{\lambda ; Q_{0}}^{\sharp, d}$ is defined by

$$
M_{\lambda ; Q_{0}}^{\sharp, d} f(x):=\sup _{Q \in \mathcal{D}\left(Q_{0}\right)} \omega_{\lambda}(f ; Q) \chi_{Q}(x), \quad\left(x \in \mathbb{R}^{n}, f \in L^{0}\left(\mathbb{R}^{n}\right)\right) .
$$

In this paper, we are interested in the following sharp maximal operator:

$$
M_{\lambda}^{\sharp, d} f(x):=\sup _{Q_{0} \in \mathcal{Q}} \sup _{Q \in \mathcal{D}\left(Q_{0}\right)} \omega_{\lambda}(f ; Q) \chi_{Q}(x) \quad\left(x \in \mathbb{R}^{n}, f \in L^{0}\left(\mathbb{R}^{n}\right)\right) .
$$

As is well known, the Fefferman-Stein sharp maximal operator defined by

$$
f^{\sharp, \eta}(x):=\sup _{Q \in \mathcal{Q}}\left(\frac{1}{|Q|} \int_{Q}\left|f(y)-f_{Q}\right|^{\eta} d y\right)^{\frac{1}{\eta}} \chi_{Q}(x),
$$

where $\eta>0$ and $f_{Q}:=\frac{1}{|Q|} \int_{Q} f(x) d x$, is a useful tool to estimate the oscillation of the function $f$. When $\eta=1$, we abbreviate $f^{\sharp, 1}$ to $f^{\sharp}$.

$$
f^{\sharp}(x):=\sup _{Q \in \mathcal{Q}} \frac{1}{|Q|} \int_{Q}\left|f(y)-f_{Q}\right| d y \times \chi_{Q}(x),
$$

There exists a close relation between these two types of the sharp maximal operators provided by Jawerth and Torchinsky in [12]:

$$
M^{(\eta)} M_{\lambda}^{\sharp, d} f(x) \sim_{\eta, \lambda} f^{\sharp, \eta}(x),
$$

for sufficiently small $\lambda$, where $M^{(\eta)}$ denotes the powered Hardy-Littlewood maximal operator defined by

$$
M^{(\eta)} f(x):=\sup _{Q \in \mathcal{Q}}\left(\frac{1}{|Q|} \int_{Q}|f(x)|^{\eta} d x\right)^{\frac{1}{\eta}} \chi_{Q}(x) .
$$

When $\eta=1, M^{(1)}$ is the original Hardy-Littlewood maximal operator.

In 18, Lerner proved the following theorem: 
Theorem $1.10\left([18)\right.$. Let $f \in L^{0}\left(\mathbb{R}^{n}\right)$ and $Q_{0} \in \mathcal{Q}$. Then there exists a sparse family of $\left\{Q_{j}^{k}\right\}_{k \in \mathbb{N}_{0}, j \in J_{k}} \subset \mathcal{D}\left(Q_{0}\right)$ such that for a.e. $x \in Q_{0}$,

$$
\left|f(x)-m_{f}\left(Q_{0}\right)\right| \leq 4 M_{\lambda_{n} ; Q_{0}}^{\sharp d} f(x)+2 \sum_{k \in \mathbb{N}_{0}} \sum_{j \in J_{k}} \omega_{\lambda_{n}}\left(f ; Q_{j}^{k}\right) \chi_{Q_{j}^{k}}(x) .
$$

Here, $\lambda_{n}:=2^{-n-2}$.

Here, we say that the family $\left\{Q_{j}^{k}\right\}_{k \in \mathbb{N}_{0}, j \in J_{k}}$ is a sparse family if the following properties hold:

(1) for each fixed $k \in \mathbb{N}_{0}$, the cubes $\left\{Q_{j}^{k}\right\}_{j \in J_{k}}$ are disjoint;

(2) if $\Omega_{k}:=\bigcup_{j \in J_{k}} Q_{j}^{k}$, then $\Omega_{k+1} \subset \Omega_{k}$;

(3) $\left|\Omega_{k+1} \cap Q_{j}^{k}\right| \leq \frac{1}{2}\left|Q_{j}^{k}\right|$ for all $j \in J_{k}$.

Now, we state our results related to the local sharp maximal operator. Throughout this paper, for $w \in A_{\infty}$, we fix the parameter $\lambda_{w}^{\prime}$ determined by $w$ so that $\lambda_{w}^{\prime}<2^{-1-2^{n+3}[w]_{A_{\infty}}}$ and let $\lambda_{w}:=2^{-n-2} \lambda_{w}^{\prime}$, where

$$
[w]_{A_{\infty}}:=\lim _{r \uparrow \infty}[w]_{A_{r}} .
$$

th-151129-3 Theorem 1.11. Let $0<s \leq q \leq p<\infty$ and $w \in A_{\infty}$.

(1) (Komori-Shirai type) For all $f \in L^{0}\left(\mathbb{R}^{n}\right)$, it holds that

$$
\|f\|_{\mathcal{M}_{q}^{p}(w, w)} \sim_{p, s, q, w}\left\|M_{\lambda_{w}}^{\sharp, d} f\right\|_{\mathcal{M}_{q}^{p}(w, w)}+\|f\|_{\mathcal{M}_{s}^{p}(w, w)} .
$$

(2) (Samko type) Additionally, we assume $w \in \mathcal{B}_{p, q}$. Then for all $f \in L^{0}\left(\mathbb{R}^{n}\right)$, it holds that

$$
\begin{aligned}
\|f\|_{\mathcal{M}_{q}^{p}(d x, w)} \quad \sim_{p, s, q, w} & \left\|M_{\lambda_{w}}^{\sharp, d} f\right\|_{\mathcal{M}_{q}^{p}(d x, w)} \\
& +\sup _{Q \in \mathcal{Q}} \Phi_{p, q, w}(Q)\left(\frac{1}{w(Q)} \int_{Q}|f(x)|^{s} d w(x)\right)^{\frac{1}{s}} .
\end{aligned}
$$

The last term in the right-hand side of (1.15) is not so artificial in view of [?, (44)]. By the Hölder inequality, it is easy to see

$$
\sup _{Q \in \mathcal{Q}} \Phi_{p, q, w}(Q)\left(\frac{1}{w(Q)} \int_{Q}|f(x)|^{s} d w(x)\right)^{\frac{1}{s}} \leq\|f\|_{\mathcal{M}_{q}^{p}(d x, w)}
$$

for all $f \in L^{0}\left(\mathbb{R}^{n}\right)$. This corresponds to the relation:

$$
\|f\|_{\mathcal{M}_{s}^{p}(w, w)} \leq\|f\|_{\mathcal{M}_{q}^{p}(w, w)}
$$

for all $f \in L^{0}\left(\mathbb{R}^{n}\right)$.

In the original sharp maximal inequality, we need to assume some integrability for $f$; see Theorem 3.8 for example. However, in Theorem 1.11, we can apply the sharp maximal inequality for any measurable functions. Note that the same refinement for the unweighted case (but the inhomogeneous setting) is obtained in 26. Theorem 1.3]; see Theorem 3.7 in the present paper for the precise formulation.

If we impose a suitable condition for $f$, then we can recover the original version of the sharp maximal inequality, see Corollaries 1.14 and 3.6 as well.

Theorem 1.12. Let $0<s \leq q \leq p<\infty, w \in A_{\infty}$. 
(1) (Komori-Shirai type) Assume that $f \in L^{0}\left(\mathbb{R}^{n}\right)$ satisfies

160121-2

$$
m_{f}\left(2^{l} Q\right) \rightarrow 0
$$

as $l \rightarrow \infty$ for any $Q \in \mathcal{Q}$ and for some medians $\left\{m_{f}\left(2^{l} Q\right)\right\}_{l \in \mathbb{N}_{0}}$. Then we have that

$$
\|f\|_{\mathcal{M}_{s}^{p}(w, w)} \lesssim_{p, s, w}\left\|M_{\lambda_{w}}^{\sharp, d} f\right\|_{\mathcal{M}_{s}^{p}(w, w)} \leq\left\|M_{\lambda_{w}^{\sharp}}^{\sharp d} f\right\|_{\mathcal{M}_{q}^{p}(w, w)} .
$$

(2) (Samko type) We assume the weighted integral condition (1.3) for $p, q$ and $w$. Then for any $f \in L^{0}\left(\mathbb{R}^{n}\right)$ satisfying (1.18) for any $Q \in \mathcal{Q}$ and for some medians $\left\{m_{f}\left(2^{l} Q\right)\right\}_{l \in \mathbb{N}}$, we have that

151215-1

$$
\|f\|_{\mathcal{M}_{q}^{p}(d x, w)} \lesssim_{p, q, w}\left\|M_{\lambda_{w}}^{\sharp, d} f\right\|_{\mathcal{M}_{q}^{p}(d x, w)} .
$$

Remark 1.13. In the case of the weighted Morrey space $\mathcal{M}_{q}^{p}(d x, w)$ of Samko type, the weighted integral condition (1.3) for $p, q$, and $w$ and the decay condition (1.18) are natural in view of the following example: In fact, if we take $0<q<p<\infty, f_{0} \equiv 1$ and $w_{0}(x):=$ $|x|^{-\frac{q}{p} n} \in A_{\infty} \cap \mathcal{B}_{p, q}$, then we know that $M f_{0} \equiv 1, f_{0}^{\sharp}=0$ and $0<\left\|M f_{0}\right\|_{\mathcal{M}_{q}^{p}\left(d x, w_{0}\right)}=$ $\left\||x|^{-\frac{n}{p}}\right\|_{\mathcal{M}_{q}^{p}(d x, d x)}<\infty$, which implies that the inequality

$$
\|f\|_{\mathcal{M}_{q}^{p}\left(d x, w_{0}\right)} \lesssim\left\|f^{\sharp}\right\|_{\mathcal{M}_{q}^{p}\left(d x, w_{0}\right)}
$$

fails even when $f$ satisfies $M f \in \mathcal{M}_{q}^{p}\left(d x, w_{0}\right)$. One can notice that for $w_{0}(x)=|x|^{-\frac{q}{p} n}$, the weighted integral condition (1.3) for $p, q$ and $w_{0}$ fails to hold; see [22].

We note that for $0<s \leq q \leq p<\infty$,

$$
\sup _{Q \in \mathcal{Q}} \Phi_{p, q, w}(Q)\left(\frac{1}{w(Q)} \int_{Q}|f(x)|^{s} d w(x)\right)^{\frac{1}{s}} \leq\|f\|_{\mathcal{M}_{q}^{p}(d x, w)}
$$

holds by Hölder's inequality. Hence, combining Theorems 1.11 and 1.12, we obtain the following:

Corollary 1.14. Let $0<q \leq p<\infty, w \in A_{\infty}$. Assume that $f \in L^{0}\left(\mathbb{R}^{n}\right)$ satisfies (1.18) for any $Q \in \mathcal{Q}$ and for some medians $\left\{m_{f}\left(2^{l} Q\right)\right\}_{l \in \mathbb{N}_{0}}$. Then

$$
\|f\|_{\mathcal{M}_{q}^{p}(w, w)} \sim_{p, q, w}\left\|M_{\lambda_{w}}^{\sharp, d} f\right\|_{\mathcal{M}_{q}^{p}(w, w)} .
$$

Moreover, if the weighted integral condition (1.3) for $p, q$ and $w$ holds, then we have that

$$
\|f\|_{\mathcal{M}_{q}^{p}(d x, w)} \sim_{p, q, w}\left\|M_{\lambda_{w}}^{\sharp, d} f\right\|_{\mathcal{M}_{q}^{p}(d x, w)} .
$$

The remaining part of this paper is organized as follows: In Section 2 we collect some important estimates. Section 3 is devoted to the proof of Theorems 1.11 and 1.12 related to the sharp maximal inequalities. Here the decomposition by Lerner [18] plays an important role. In Section 4. we prove Theorems 1.3 and 1.8 and then give another application of the sharp maximal inequalities. We take up the boundedness of the commutators.

\section{Preliminaries}

We first recall an important property of the class $\mathcal{B}_{p, q}$ and the relation between (1.7) and $M$.

lm-160626-3 Lemma 2.1 (22, 30]). Let $0<q \leq p<\infty$ and $w$ be a weight.

(1) Then $w \in \mathcal{B}_{p, q}$ if and only if $\left\|\chi_{Q_{0}}\right\|_{\mathcal{M}_{q}^{p}(d x, w)} \sim \Phi_{p, q, w}\left(Q_{0}\right)$ for any $Q_{0} \in \mathcal{Q}$; see (1.2).

(2) We assume $q>1$ further. If $M$ is bounded on $\mathcal{M}_{q}^{p}(d x, w)$, then (1.7) holds. 
We also prove an important relation combining the $\mathcal{B}_{p, q}$ condition and (1.7) as follows:

lm-160113-1 Lemma 2.2. Let $0<q \leq p<\infty$ and $w$ be a weight.

aa

160113-4

$\mathrm{bb}$

$160113-5$

(1) $w$ satisfies (1.7) for all $Q \in \mathcal{Q}$, if and only if $w \in \mathcal{B}_{p, q}$ and

$$
\frac{1}{|Q|} \Phi_{p, q, w}(Q)\left\|w^{-\frac{1}{q}} \chi_{Q}\right\|_{H^{q^{\prime}, n(1-q / p)}} \leq C_{0}
$$

holds for all $Q \in \mathcal{Q}$.

(2) For fixed $Q \in \mathcal{Q}, w$ satisfies (2.1), if and only if

$$
\frac{1}{|Q|} \int_{Q} f(x) d x \leq C_{0} \frac{\left\|f \cdot \chi_{Q}\right\|_{\mathcal{M}_{q}^{p}(d x, w)}}{\Phi_{p, q, w}(Q)}
$$

holds for any $f \geq 0$. Here, the constant $C_{0}$ in (2.2) is the same as the one of (2.1). Moreover, once $w$ satisfies (2.2) for all $Q$ with $C_{0}$ independent of $Q$, then $w$ is in the class $A_{q+1}$. In particular, $w$ is a doubling weight.

Remark 2.3. From Lemma 2.2 particularly, we see that the inequality (1.7) implies $w \in$ $\mathcal{B}_{p, q} \cap A_{q+1}$.

Proof. To show the assertion (11), we have only to prove $w \in \mathcal{B}_{p, q}$ by imposing (1.7) for all $Q \in \mathcal{Q}$. The converse is clear from the definition of $\Phi_{p, q, w}$. To this end, we recall the notion of the sparse family. Fix any $Q_{0} \in \mathcal{D}$ and let us show that

$$
\left\|\chi_{Q_{0}}\right\|_{\mathcal{M}_{q}^{p}(d x, w)} \sim_{n, p, q, w} \Phi_{p, q, w}\left(Q_{0}\right) .
$$

Set $\gamma_{0}:=\frac{w\left(Q_{0}\right)}{\left|Q_{0}\right|}$ and take $a \gg 2^{n}$. Define $\mathcal{D}_{0}:=\left\{Q_{0}\right\}$ and

151215-2

$$
\mathcal{D}_{k}:=\left\{Q \in \mathcal{D}\left(Q_{0}\right): \frac{w(Q)}{|Q|}>a^{k} \gamma_{0}\right\} \quad(k \in \mathbb{N}) .
$$

By $\mathcal{D}_{k}^{*}:=\left\{Q_{j}^{k}\right\}_{j \in J_{k}}$, we denote the maximal subset of $\mathcal{D}_{k}$. The maximality implies that for each $Q_{j}^{k} \in \mathcal{D}_{k}^{*}$,

$$
a^{k} \gamma_{0}<\frac{w\left(Q_{j}^{k}\right)}{\left|Q_{j}^{k}\right|} \leq 2^{n} a^{k} \gamma_{0} .
$$

Then we claim that the family $\left\{Q_{j}^{k}\right\}_{k \in \mathbb{N}_{0}, i \in J_{k}}$ is a sparse family. By the maximality, it is clear that $\left\{Q_{j}^{k}\right\}_{j \in J_{k}}$ is pairwise disjointed. Moreover, for any $Q_{j}^{k+1} \in \mathcal{D}_{k+1}^{*}$, it follows from the definition of $\mathcal{D}_{k+1}$ that $\frac{w\left(Q_{j}^{k+1}\right)}{\left|Q_{j}^{k+1}\right|}>a^{k} \gamma_{0}$ by $a>1$, which implies $Q_{j}^{k+1} \in \mathcal{D}_{k}$. Namely, we have $\Omega_{k+1} \subset \Omega_{k}$, where we denote

$$
\Omega_{k}:=\bigcup_{j \in J_{k}} Q_{j}^{k}
$$

We also have that

$$
\left|Q_{j}^{k} \cap \Omega_{k+1}\right| \leq \frac{2^{n}}{a}\left|Q_{j}^{k}\right|,
$$

for any $k \in \mathbb{N}_{0}$ and $j \in J_{k}$. Indeed, since $\left\{Q_{i}^{k+1}\right\}_{i \in J_{k+1}}$ is disjoint, thanks to (2.4)

$$
\begin{aligned}
&\left|Q_{j}^{k} \cap \Omega_{k+1}\right|=\sum_{i \in J_{k+1}: Q_{j}^{k+1} \subset Q_{j}^{k}}\left|Q_{i}^{k+1}\right| \\
& \leq \sum_{i \in J_{k+1}: Q_{j}^{k+1} \subset Q_{j}^{k}} \frac{1}{a^{k+1} \gamma_{0}} w\left(Q_{i}^{k+1}\right) \\
& \leq \frac{1}{a^{k+1} \gamma_{0}} w\left(Q_{j}^{k}\right) \leq \frac{2^{n}}{a}\left|Q_{j}^{k}\right| . \\
& 8
\end{aligned}
$$


Now, it follows from (2.6) that $\frac{\left|Q_{j}^{k} \backslash \Omega_{k+1}\right|}{\left|Q_{j}^{k}\right|} \geq 2^{-1}$, for all $k \in \mathbb{N}_{0}$ and $j \in J_{k}$. In particular, we focus on the case of $k=0$ to obtain that $\frac{1}{\left|Q_{0}\right|} \int_{Q_{0}} \chi_{Q_{0} \backslash \Omega_{1}}(x) d x \geq 2^{-1}$. Using the dual inequality (1.5) and $a \gg 1$, we see that

$$
\frac{1}{2} \leq \frac{1}{\left|Q_{0}\right|}\left\|\chi_{Q_{0} \backslash \Omega_{1}}\right\|_{\mathcal{M}_{q}^{p}(d x, w)}\left\|w^{-\frac{1}{q}} \chi_{Q_{0}}\right\|_{H^{q^{\prime}, n(1-q / p)}} .
$$

Moreover, it follows from the assumption (1.7) that

$$
\left\|\chi_{Q_{0}}\right\|_{\mathcal{M}_{q}^{p}(d x, w)} \leq C\left\|\chi_{Q_{0} \backslash \Omega_{1}}\right\|_{\mathcal{M}_{q}^{p}(d x, w)} .
$$

Now, let us recall that

$$
\begin{aligned}
& \left\|\chi_{Q_{0} \backslash \Omega_{1}}\right\|_{\mathcal{M}_{q}^{p}(d x, w)}=\sup _{R \in \mathcal{D}\left(Q_{0}\right)}|R|^{\frac{1}{p}}\left(\frac{w\left(R \backslash \Omega_{1}\right)}{|R|}\right)^{\frac{1}{q}}, \\
& \Omega_{1}=\bigcup_{R \in \mathcal{D}_{1}} R, \quad \mathcal{D}_{1}=\left\{R \in \mathcal{D}\left(Q_{0}\right): \frac{w(R)}{|R|}>a \frac{w\left(Q_{0}\right)}{\left|Q_{0}\right|}\right\} .
\end{aligned}
$$

This implies that for any $R \in \mathcal{D}\left(Q_{0}\right) \backslash \mathcal{D}_{1}, w\left(R \backslash \Omega_{1}\right)=0$ and hence,

$$
\left\|\chi_{Q_{0} \backslash \Omega_{1}}\right\|_{\mathcal{M}_{q}^{p}(d x, w)}=\sup _{R \in \mathcal{D}\left(Q_{0}\right) \backslash \mathcal{D}_{1}}|R|^{\frac{1}{p}}\left(\frac{w\left(R \backslash \Omega_{1}\right)}{|R|}\right)^{\frac{1}{q}} \leq a^{\frac{1}{q}} \Phi_{p, q, w}\left(Q_{0}\right) .
$$

In summary, we obtain that

$$
\left\|\chi_{Q_{0}}\right\|_{\mathcal{M}_{q}^{p}(d x, w)} \leq C_{a, n, p, q, w} \Phi_{p, q, w}\left(Q_{0}\right) .
$$

Meanwhile, since the converse inequality is trivial, it follows $\left\|\chi_{Q_{0}}\right\|_{\mathcal{M}_{q}^{p}(d x, w)} \sim \Phi_{p, q, w}\left(Q_{0}\right)$ and hence $w \in \mathcal{B}_{p, q}$.

Next, let us show the assertion (2). Once we suppose (2.1), then we deduce (2.2) immediately from (1.5). Conversely, if we assume (2.2), then we employ (1.6) to obtain

$$
\begin{aligned}
\left\|w^{-\frac{1}{q}} \chi_{Q}\right\|_{H^{q^{\prime}, n(1-q / p)}} & =\sup _{f \geq 0:\left\|f \chi_{Q}\right\|_{\mathcal{M}_{q}^{p}(d x, w)} \leq 1} \int_{Q} f(x) d x \\
& \leq \sup _{f \geq 0:\left\|f \chi_{Q}\right\|_{\mathcal{M}_{q}^{p}(d x, w)} \leq 1} C_{0} \frac{|Q|}{\Phi_{p, q, w}(Q)}\left\|f \chi_{Q}\right\|_{\mathcal{M}_{q}^{p}(d x, w)} \\
& =C_{0} \frac{|Q|}{\Phi_{p, q, w}(Q)},
\end{aligned}
$$

which implies (2.1).

Finally, we shall show that $w$ is in $A_{q+1}$ by imposing (2.2) for all $Q \in \mathcal{Q}$. Note that

$$
\left\|w^{-\frac{1}{q}} \chi_{Q}\right\|_{\mathcal{M}_{q}^{p}(d x, w)}=\left\|\chi_{Q}\right\|_{\mathcal{M}_{q}^{p}(d x, d x)}=|Q|^{\frac{1}{p}} .
$$

With this in mind, by putting $f:=w^{-\frac{1}{q}}$ in (2.2), then it follows that

$$
\frac{1}{|Q|} \int_{Q} w(x)^{-\frac{1}{q}} d x \leq C_{0} \frac{|Q|^{\frac{1}{p}}}{\Phi_{p, q, w}(Q)}=C_{0}\left(\frac{|Q|}{w(Q)}\right)^{\frac{1}{q}},
$$

which implies that $[w]_{A_{q+1}} \leq C_{0}$.

In 21, Nakai quantitatively showed the self-improvement of the integral condition; see 23, Proposition 2.6] as well. 
pr-160115-1 Proposition 2.4 (21]). Assume that a positive function $f:(0, \infty) \rightarrow(0, \infty)$ satisfies the integral condition:

$160115-1$

$$
\int_{1}^{\infty} \frac{1}{f(r s)} \frac{d s}{s} \leq \frac{C_{0}}{f(r)} \quad(r>0)
$$

for some constant $C_{0}>0$. Then for all $\delta \in\left(0, C_{0}^{-1}\right)$, we have

$160115-2$

$$
\int_{1}^{\infty} \frac{s^{\delta}}{f(r s)} \frac{d s}{s} \leq \frac{C_{0}}{1-C_{0} \delta} \frac{1}{f(r)} \quad(r>0) .
$$

Using Proposition 2.4 we can show the self-improvement of the weighted integral condition. We will invoke the following observation in the proof of Theorem 1.8

Im-160115-1 Lemma 2.5. Let $0<q \leq p<\infty$ and $w$ satisfies the weighted integral condition (1.3) for $p, q$ and $w$. Then we have

$$
\int_{1}^{\infty} \frac{\log s}{\Phi_{p, q, w}(s Q)} \frac{d s}{s} \leq \frac{C}{\Phi_{p, q, w}(Q)} \quad(Q \in \mathcal{Q})
$$

or equivalently,

$$
\sum_{k=1}^{\infty} \frac{k}{\Phi_{p, q, w}\left(2^{k} Q\right)} \leq \frac{C}{\Phi_{p, q, w}(Q)} \quad(Q \in \mathcal{Q})
$$

Proof. Fix any cube $Q$. We put $f(s):=\Phi_{p, q, w}(s Q)$ for $s \geq 1$ and $f(s):=\Phi_{p, q, w}(Q)$ for $s \leq 1$. Since we assume the weighted integral condition (1.3) for $p, q$ and $w$, the integral condition for the above $f$ holds. Hence, we see that by Proposition 2.4

$$
\int_{1}^{\infty} \frac{s^{\delta}}{\Phi_{p, q, w}(s Q)} \frac{d s}{s} \leq \frac{C}{\Phi_{p, q, w}(Q)}
$$

for some $\delta>0$. If we notice that $\log s \leq C_{\delta} s^{\delta}$, then we obtain (2.9). Note that the equivalence between (2.9) and (2.10) is clear from this observation.

Next, we collect the fundamental properties of the median and oscillation; see [10] for the details. Let $f \in L^{0}\left(\mathbb{R}^{n}\right), \lambda \in\left(0,2^{-1}\right)$ and $Q \in \mathcal{Q}$. For any median $m_{f}(Q)$, we have that

$$
\left|m_{f}(Q)\right| \leq\left(f \cdot \chi_{Q}\right)^{*}(\lambda|Q|), \quad \lim _{\ell(Q) \rightarrow 0, Q \ni x} m_{f}(Q)=f(x) \quad\left(\text { a.e. } x \in \mathbb{R}^{n}\right) .
$$

See [5, Lemma 2.2] for the details. Moreover, for the oscillation of $f$, we have that

$$
\omega_{\lambda}(f ; Q) \leq\left(\left(f-m_{f}(Q)\right) \chi_{Q}\right)^{*}(\lambda|Q|) \leq 2 \omega_{\lambda}(f ; Q) .
$$

Now, we introduce the notion of $w$-sparse family for $w \in A_{\infty}$. Let $w \in A_{\infty}$ and $\lambda_{w}^{\prime}<$

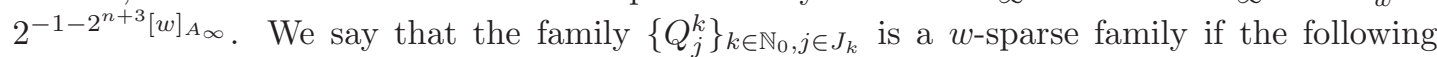
properties hold:

(1) for each fixed $k \in \mathbb{N}_{0}$, the cubes $\left\{Q_{j}^{k}\right\}_{j \in J_{k}}$ are disjoint;

(2) if $\Omega_{k}:=\bigcup_{j \in J_{k}} Q_{j}^{k}$, then $\Omega_{k+1} \subset \Omega_{k}$;

(3) $\left|\Omega_{k+1} \cap Q_{j}^{k}\right| \leq \lambda_{w}^{\prime}\left|Q_{j}^{k}\right|$ for all $j \in J_{k}$.

The only difference from the original definition of the sparse family is the constant $\lambda_{w}^{\prime}$ appearing in the condition (3). That is, if we replace the constant $\lambda_{w}^{\prime}$ by $2^{-1}$ in the condition (3), then the $w$-sparse family turns into the original sparse family introduced in [10. The advantage of $w$-sparse family is as follows: 
lm-151201 Lemma 2.6. Let $w \in A_{\infty}, \lambda_{w}^{\prime}<2^{-1-2^{n+3}[w]_{\infty}}$ and $\left\{Q_{j}^{k}\right\}_{k \in \mathbb{N}_{0}, j \in J_{k}}$ be a w-sparse family. Then there exists a constant $C_{w}>1$ such that

$$
w\left(Q_{j}^{k}\right) \leq C_{w} w\left(Q_{j}^{k} \cap \Omega_{k+1}^{c}\right),
$$

holds for all $k \in \mathbb{N}_{0}$ and $j \in J_{k}$. Here, the constant $C_{w}$ can be written by

$$
C_{w}=\left(1-2\left(\lambda_{w}^{\prime}\right)^{\frac{\varepsilon}{1+\varepsilon}}\right)^{-1}, \quad \varepsilon:=\frac{1}{2^{n+3}[w]_{A_{\infty}}} .
$$

We can find such a property in many papers; see for example [6], and hence, we omit the proof. We will invoke Lemma 2.6 to show Theorem 3.2 which is a crucial part of Theorem 1.11.

Here, we give an observation combining our results and the original sharp maximal inequalities. We will employ the following lemma to show Corollary 3.6.

lm-151201-3 Lemma 2.7. Let $f \in L^{0}\left(\mathbb{R}^{n}\right)$. Assume that $M f \in \mathcal{M}_{q_{0}}^{p_{0}}\left(v_{0}, w_{0}\right)$ for some $0<q_{0} \leq p_{0}<\infty$ and some weights $v_{0}, w_{0}$ satisfying that

$151203-1$

$$
\lim _{l \rightarrow \infty} \Phi_{p_{0}, q_{0}, v_{0}, w_{0}}\left(2^{l} Q\right)=\infty, \quad(Q \in \mathcal{Q}), \quad \Phi_{p_{0}, q_{0}, v_{0}, w_{0}}(Q):=v_{0}(Q)^{\frac{1}{p_{0}}-\frac{1}{q_{0}}} w_{0}(Q)^{\frac{1}{q_{0}}}
$$

Then for any $Q \in \mathcal{Q}$ and any medians $\left\{m_{f}\left(2^{l} Q\right)\right\}_{l \in \mathbb{N}_{0}}$, it holds that

$$
\lim _{l \rightarrow \infty} m_{f}\left(2^{l} Q\right)=0 \text {. }
$$

In particular, we have the following:

(1) If we assume that $M f \in \mathcal{M}_{q_{0}}^{p_{0}}\left(w_{0}, w_{0}\right)$ for some $0<q_{0} \leq p_{0}<\infty$ and $w_{0} \in A_{\infty}$. Then for any $Q \in \mathcal{Q}$ and any medians $\left\{m_{f}\left(2^{l} Q\right)\right\}_{l \in \mathbb{N}_{0}}$, 2.14 holds.

(2) If we assume that $M f \in \mathcal{M}_{q_{0}}^{p_{0}}\left(d x, w_{0}\right)$ for some $0<q_{0} \leq p_{0}<\infty$ and $w_{0} \in A_{\infty}$ satisfying the weighted integral condition (1.3) for $p_{0}, q_{0}$ and $w_{0}$. Then for any $Q \in \mathcal{Q}$ and any medians $\left\{m_{f}\left(2^{l} Q\right)\right\}_{l \in \mathbb{N}_{0}}$, 2.14) holds.

Proof. In view of (2.11), for $\lambda \in\left(0,2^{-1}\right)$, we have that

$$
\left|m_{f}\left(2^{l} Q\right)\right| \leq\left(f \cdot \chi_{2^{l} Q}\right)^{*}\left(\lambda\left|2^{l} Q\right|\right) \leq \frac{1}{\lambda\left|2^{l} Q\right|} \int_{0}^{\infty}\left(f \cdot \chi_{2^{l} Q}\right)^{*}(t) d t \leq \frac{1}{\lambda} \inf _{x \in 2^{l} Q} M f(x) .
$$

With the definition of the norm in mind, by taking $\lambda=4^{-1}$ for example, we notice that

$$
\left|m_{f}\left(2^{l} Q\right)\right| \lesssim \frac{1}{w_{0}\left(2^{l} Q\right)^{\frac{1}{q_{0}}}}\left(\int_{2^{l} Q} M f(x)^{q_{0}} d w_{0}(x)\right)^{\frac{1}{q_{0}}} \leq \frac{\|M f\|_{\mathcal{M}_{q_{0}}^{p_{0}}\left(v_{0}, w_{0}\right)}}{\Phi_{p_{0}, q_{0}, v_{0}, w_{0}}\left(2^{l} Q\right)}
$$

By our assumption, $\|M f\|_{\mathcal{M}_{q_{0}}^{p_{0}}\left(v_{0}, w_{0}\right)}<\infty$. Hence, the assumption (2.13) yields the conclusion (2.14).

Particularly, let us consider the case of $v_{0}=w_{0} \in A_{\infty}$. The condition (2.13) reads $\lim _{l \rightarrow \infty} w_{0}\left(2^{l} Q\right)^{\frac{1}{p_{0}}}=\infty$ for all $Q \in \mathcal{Q}$. Note that $w_{0} \in A_{\infty}$ implies $w_{0} \notin L^{1}(d x)$; see [29. Chapter $1, \S 8.6(\mathrm{a})]$. In fact, by the reverse Hölder inequality, for any $R>0$, we have that

$$
\left(\frac{1}{|Q(R)|} \int_{Q(R)} w_{0}(x)^{1+\varepsilon} d x\right)^{\frac{1}{1+\varepsilon}} \leq \frac{2 w_{0}(Q(R))}{|Q(R)|}
$$

which implies

$$
|Q(R)|^{1-\frac{1}{1+\varepsilon}}\left(\int_{Q(R)} w_{0}(x)^{1+\varepsilon} d x\right)^{\frac{1}{1+\varepsilon}} \leq 2 w_{0}(Q(R))
$$


By tending $R \rightarrow \infty$, we obtain $\left\|w_{0}\right\|_{L^{1}(d x)}=\infty$. Thus, we see that $\lim _{l \rightarrow \infty} w_{0}\left(2^{l} Q\right)^{\frac{1}{p}}=$ $\left\|w_{0}\right\|_{L^{1}(d x)}^{\frac{1}{p}}=\infty$.

In the case of $v_{0}=d x$, the condition (2.13) reads $\lim _{l \rightarrow \infty} \Phi_{p_{0}, q_{0}, w_{0}}\left(2^{l} Q\right)=\infty$ for all $Q \in \mathcal{Q}$. However, this condition follows directly from the weighted integral condition (1.3) for $p_{0}, q_{0}$ and $w_{0}$.

Remark 2.8. The assumption (2.13) is essential in the following sense: If the assumption (2.13) does not hold, then $M f=f \equiv 1$ is in $\mathcal{M}_{q_{0}}^{p_{0}}\left(v_{0}, w_{0}\right)$, but (2.14) fails for any $Q \in \mathcal{Q}$.

To ensure the well definedness of the singular integral operators and commutators on weighted Morrey spaces in Theorems 1.3 and 1.8 we need the self-improvement property of the boundedness of $M$ on $\mathcal{M}_{q}^{p}(d x, w)$. The self-improvement property of $M$ on the Banach function spaces is obtained in [19, Theorem 1.2].

Lm-160113-2 Lemma 2.9. Let $1<q \leq p<\infty$ and $w$ be a weight. Assume that the Hardy-Littlewood maximal operator $M$ is bounded on $\mathcal{M}_{q}^{p}(d x, w)$. Then there exists $r=r(p, q, w)>1$ such that $M^{(r)}$ is also bounded on $\mathcal{M}_{q}^{p}(d x, w)$.

Since the proof of Lemma 2.9 is almost the same as the one of [19, Theorem 1.2], we omit the proof here. We can rephrase the assertion of Lemma 2.9 as follows: Once we obtain the boundedness of $M$ on $\mathcal{M}_{q}^{p}(d x, w)$ for some $1<q \leq p<\infty$ and some weight $w$, then there exists a small $\varepsilon \in(0,1)$ such that $M$ is also bounded on $\mathcal{M}_{q(1-\varepsilon)}^{p(1-\varepsilon)}(d x, w)$. This property yields the following corollary:

cr-160113-4 Corollary 2.10. Assume that the Hardy-Littlewood maximal operator $M$ is bounded on $\mathcal{M}_{q}^{p}(d x, w)$ for some $1<q \leq p<\infty$ and some weight $w$. Then for any $Q \in \mathcal{Q}$ and any $f \in \mathcal{M}_{q}^{p}(d x, w)$, $f \cdot \chi_{Q}$ is in $L^{\frac{1}{1-\varepsilon}}\left(\mathbb{R}^{n}\right)$.

Proof. Let $\varepsilon$ be as above. In this proof, we abbreviate $p(1-\varepsilon)$ and $q(1-\varepsilon)$ by $p_{\varepsilon}$ and $q_{\varepsilon}$ respectively. By the above observation, we know that $M$ is bounded on $\mathcal{M}_{q_{\varepsilon}}^{p_{\varepsilon}}(d x, w)$. By Lemmas 2.1 and 2.2, it follows that

$$
\frac{1}{|Q|} \Phi_{p, q, w}(Q)^{\frac{1}{1-\varepsilon}}\left\|w^{-\frac{1}{q_{\varepsilon}}} \chi_{Q}\right\|_{H_{q_{\varepsilon}^{\prime}, n(1-q / p)}} \lesssim 1
$$

for all $Q \in \mathcal{Q}$. Here, we used a simple fact that $\Phi_{p_{\varepsilon}, q_{\varepsilon}, w}(Q)=\Phi_{p, q, w}(Q)^{\frac{1}{1-\varepsilon}}$. With this in mind, by invoking the dual inequality (1.5), we have that

$$
\begin{aligned}
\int_{\mathbb{R}^{n}}\left|f(x) \chi_{Q}(x)\right|^{\frac{1}{1-\varepsilon}} d x & \leq C\left\||f|^{\frac{1}{1-\varepsilon}} w^{\frac{1}{q_{\varepsilon}}}\right\|_{\mathcal{M}_{q_{\varepsilon}}^{p_{\varepsilon}}(d x, d x)}\left\|w^{-\frac{1}{q_{\varepsilon}}} \chi_{Q}\right\|_{H^{q_{\varepsilon}^{\prime}, n(1-q / p)}} \\
& \leq C\left\||f|^{\frac{1}{1-\varepsilon}}\right\|_{\mathcal{M}_{q_{\varepsilon}}^{p_{\varepsilon}}(d x, w)} \frac{|Q|}{\Phi_{p, q, w}(Q)^{\frac{1}{1-\varepsilon}}} .
\end{aligned}
$$

If we notice that $\left\||f|^{\frac{1}{1-\varepsilon}}\right\|_{\mathcal{M}_{q \varepsilon}^{p_{\varepsilon}}(d x, w)}=\|f\|_{\mathcal{M}_{q}^{p}(d x, w)}^{\frac{1}{1-\varepsilon}}$, then we see that

$$
\left\|f \cdot \chi_{Q}\right\|_{L^{\frac{1}{1-\varepsilon}}\left(\mathbb{R}^{n}\right)} \leq C \frac{|Q|^{1-\varepsilon}}{\Phi_{p, q, w}(Q)}\|f\|_{\mathcal{M}_{q}^{p}(d x, w)}<\infty .
$$


Our first observation is to generalize Theorem 1.10 to the $w$-sparse family setting.

Proposition 3.1. Let $w \in A_{\infty}, \lambda_{w}^{\prime}<2^{-1-2^{n+3}[w]_{A_{\infty}}}$ and $\lambda_{w}:=2^{-n-2} \lambda_{w}^{\prime}$. For $Q_{0} \in \mathcal{Q}$ and $f: Q_{0} \rightarrow \mathbb{R}$, there exists a w-sparse family $\left\{Q_{j}^{k}\right\}_{k \in \mathbb{N}_{0}, j \in J_{k}} \subset \mathcal{D}\left(Q_{0}\right)$ such that for a.e. $x \in \mathbb{R}^{n}$,

$$
\left|f(x)-m_{f}\left(Q_{0}\right)\right| \leq 4 M_{\lambda_{w} ; Q_{0}}^{\sharp, d} f(x)+2 \sum_{k \in \mathbb{N}_{0}} \sum_{j \in J_{k}} \omega_{\lambda_{w}}\left(f ; Q_{j}^{k}\right) \chi_{Q_{j}^{k}}(x) .
$$

Since we need only a slight modification of the proof of Theorem 1.10 to show Proposition 3.1. we omit the proof here. By employing Proposition 3.1 we can show the following local estimate:

th-151129-2 Theorem 3.2. Let $0<q, s<\infty, w, v \in A_{\infty}$ and $Q_{0} \in \mathcal{Q}$. We choose $\lambda_{w}^{\prime}>0$ so that

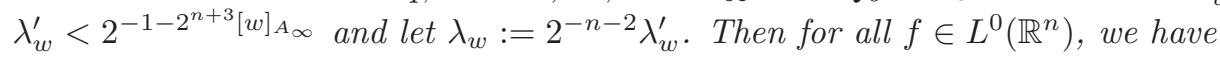

$$
\begin{aligned}
\left(\int_{Q_{0}}|f(x)|^{q} d w(x)\right)^{\frac{1}{q}} \lesssim_{q, s, w, v} & \left(\int_{Q_{0}} M_{\lambda_{w} ; Q_{0}}^{\sharp, d} f(x)^{q} d w(x)\right)^{\frac{1}{q}} \\
& +w\left(Q_{0}\right)^{\frac{1}{q}}\left(\frac{1}{v\left(Q_{0}\right)} \int_{Q_{0}}|f(x)|^{s} d v(x)\right)^{\frac{1}{s}} .
\end{aligned}
$$

Proof. We take a median $m_{f}\left(Q_{0}\right)$ and use the quasi-triangle inequality to get

$$
\left(\int_{Q_{0}}|f(x)|^{q} d w(x)\right)^{\frac{1}{q}} \lesssim_{q}\left(\int_{Q_{0}}\left|f(x)-m_{f}\left(Q_{0}\right)\right|^{q} d w(x)\right)^{\frac{1}{q}}+w\left(Q_{0}\right)^{\frac{1}{q}}\left|m_{f}\left(Q_{0}\right)\right|
$$

For the first term, by applying Proposition 3.1 we obtain that

$$
\begin{gathered}
\left(\int_{Q_{0}}\left|f(x)-m_{f}\left(Q_{0}\right)\right|^{q} d w(x)\right)^{\frac{1}{q}} \\
\lesssim q\left(\int_{Q_{0}} M_{\lambda_{w} ; Q_{0}}^{\sharp, d} f(x)^{q} d w(x)\right)^{\frac{1}{q}}+\left[\int_{Q_{0}}\left(\sum_{k \in \mathbb{N}_{0}} \sum_{j \in J_{k}} \omega_{\lambda_{w}}\left(f ; Q_{j}^{k}\right) \chi_{Q_{j}^{k}}(x)\right)^{q} d w(x)\right]^{\frac{1}{q}} .
\end{gathered}
$$

Now, we focus on

$$
\mathrm{I}:=\left[\int_{Q_{0}}\left(\sum_{k \in \mathbb{N}_{0}} \sum_{j \in J_{k}} \omega_{\lambda_{w}}\left(f ; Q_{j}^{k}\right) \chi_{Q_{j}^{k}}(x)\right)^{q} d w(x)\right]^{\frac{1}{q}}
$$

We recall that $\left\{Q_{j}^{k}\right\}_{k \in \mathbb{N}_{0}, j \in J_{k}}$ is a $w$-sparse family, in particular, $w\left(Q_{j}^{k}\right) \leq C_{w} w\left(Q_{j}^{k} \cap \Omega_{k+1}^{c}\right)$ holds by Lemma 2.6. where $\Omega_{k+1}$ is given by (2.5). This implies that $\chi_{Q_{j}^{k}}(x) \leq C_{w} M_{w}\left[\chi_{Q_{j}^{k} \cap \Omega_{k+1}^{c}}\right](x)$, where the weighted Hardy-Littlewood maximal operator $M_{w}$ is defined by

$$
M_{w} f(x):=\sup _{Q \in \mathcal{Q}} \frac{\chi_{Q}(x)}{w(Q)} \int_{Q}|f(y)| d w(y)
$$


Hence, by taking $\eta>\max \left(1, q^{-1}\right)$ and employing the boundedness of $M_{w}$ on $L^{\eta q}\left(\ell^{\eta}\right)(w)$, it follows that

$$
\begin{aligned}
\mathrm{I} & \lesssim_{w}\left[\int_{Q_{0}}\left(\sum_{k \in \mathbb{N}_{0}} \sum_{j \in J_{k}} \omega_{\lambda_{w}}\left(f ; Q_{j}^{k}\right) M_{w}\left[\chi_{Q_{j}^{k} \cap \Omega_{k+1}^{c}}\right](x)^{\eta}\right)^{q} d w(x)\right]^{\frac{1}{q}} \\
& \lesssim_{q, w}\left[\int_{\mathbb{R}^{n}}\left(\sum_{k \in \mathbb{N}_{0}} \sum_{j \in J_{k}} \omega_{\lambda_{w}}\left(f ; Q_{j}^{k}\right) \chi_{Q_{j}^{k} \cap \Omega_{k+1}^{c}}(x)\right)^{q} d w(x)\right]^{\frac{1}{q}} .
\end{aligned}
$$

If we notice that $\omega_{\lambda_{w}}\left(f ; Q_{j}^{k}\right) \chi_{Q_{j}^{k}}(x) \leq M_{\lambda_{w} ; Q_{0}}^{\sharp, d} f(x)$ and that $\sum_{k \in \mathbb{N}_{0}} \sum_{j \in J_{k}} \chi_{Q^{k_{j}} \cap \Omega_{k+1}^{c}} \leq \chi_{Q_{0}}$ by the disjointness of $\left\{Q_{j}^{k} \cap \Omega_{k+1}^{c}\right\}_{k \in \mathbb{N}_{0}, j \in J_{k}}$, then we see that

$$
\mathrm{I} \lesssim_{q, w}\left(\int_{Q_{0}} M_{\lambda_{w} ; Q_{0}}^{\sharp, d} f(x)^{q} d w(x)\right)^{\frac{1}{q}} .
$$

Next, we evaluate the second term: $w\left(Q_{0}\right)^{\frac{1}{q}}\left|m_{f}\left(Q_{0}\right)\right|$. Since $v \in A_{\infty}$, we can find $u \in(1, \infty)$ such that $v \in A_{u}$. We set $r:=\frac{s}{u}$. By (2.11), for $\lambda \in\left(0,2^{-1}\right)$, we have that

$$
\begin{aligned}
\left|m_{f}\left(Q_{0}\right)\right| & \leq\left(f \cdot \chi_{Q_{0}}\right)^{*}\left(\lambda\left|Q_{0}\right|\right) \\
& \leq\left(\frac{1}{\lambda\left|Q_{0}\right|} \int_{0}^{\lambda\left|Q_{0}\right|}\left(f \cdot \chi_{Q_{0}}\right)^{*}(t)^{r} d t\right)^{\frac{1}{r}} \\
& \lesssim_{r}\left(\frac{1}{\left|Q_{0}\right|} \int_{Q_{0}}|f(x)|^{r} d x\right)^{\frac{1}{r}} .
\end{aligned}
$$

Moreover, by $v \in A_{u}$ and $r u=s$, we see that

$$
\begin{aligned}
\left|m_{f}\left(Q_{0}\right)\right| & \lesssim r \quad[v]_{A_{u}}^{\frac{1}{r u}}\left(\frac{1}{\left|Q_{0}\right|} \int_{Q_{0}}|f(x)|^{r u} d v(x)\right)^{\frac{1}{r u}} \cdot\left(\frac{\left|Q_{0}\right|}{v\left(Q_{0}\right)}\right)^{\frac{1}{r u}} \\
& =[v]_{A_{u}}^{\frac{1}{s}}\left(\frac{1}{v\left(Q_{0}\right)} \int_{Q_{0}}|f(x)|^{s} d v(x)\right)^{\frac{1}{s}} .
\end{aligned}
$$

Thus, we complete the proof.

As a corollary, we obtain the following:

Corollary 3.3. Let $0<q \leq p<\infty, s>0, w_{1}$ be a weight, and $w_{2}, v \in A_{\infty}$. Then for any $f \in L^{0}\left(\mathbb{R}^{n}\right)$, one has

$$
\begin{aligned}
\|f\|_{\mathcal{M}_{q}^{p}\left(w_{1}, w_{2}\right)} \lesssim \lesssim_{q, s, w_{2}, v} & \left\|M_{\lambda_{w}}^{\sharp, d} f\right\|_{\mathcal{M}_{q}^{p}\left(w_{1}, w_{2}\right)} \\
& +\sup _{Q \in \mathcal{Q}} w_{1}(Q)^{\frac{1}{p}}\left(\frac{w_{2}(Q)}{w_{1}(Q)}\right)^{\frac{1}{q}}\left(\frac{1}{v(Q)} \int_{Q}|f(x)|^{s} v(x) d x\right)^{\frac{1}{s}} .
\end{aligned}
$$

In particular, the following two types of weighted sharp maximal inequalities hold:

(1) (Komori-Shirai type: $w_{1}=w_{2}=v=w \in A_{\infty}$ )

$$
\|f\|_{\mathcal{M}_{q}^{p}(w, w)} \lesssim_{s, q, w}\left\|M_{\lambda_{w}^{\sharp}}^{\sharp d} f\right\|_{\mathcal{M}_{q}^{p}(w, w)}+\|f\|_{\mathcal{M}_{s}^{p}(w, w)} .
$$


(2) (Samko type: $w_{1}=d x, w_{2}=v=w \in A_{\infty}$ )

$$
\begin{aligned}
& \|f\|_{\mathcal{M}_{q}^{p}(d x, w)} \\
& \quad \lesssim_{s, q, w} \quad\left\|M_{\lambda_{w}}^{\sharp, d} f\right\|_{\mathcal{M}_{q}^{p}(d x, w)}+\sup _{Q \in \mathcal{Q}} \Phi_{p, q, w}(Q)\left(\frac{1}{w(Q)} \int_{Q}|f(x)|^{s} d w(x)\right)^{\frac{1}{s}} .
\end{aligned}
$$

Next, we consider the converse inequality. To this end, we recall the sufficient conditions for the boundedness of $M$ on weighted Morrey spaces.

Theorem 3.4. Let $1<q \leq p<\infty$ and $w \in A_{q}$.

(1) [15] We have that $\|M f\|_{\mathcal{M}_{q}^{p}(w, w)} \lesssim_{p, q, w}\|f\|_{\mathcal{M}_{q}^{p}(w, w)}$ for all $\left.f \in \mathcal{M}_{q}^{p}(w, w)\right)$.

(2) [22] In addition, we assume $w \in \mathcal{B}_{p, q}$. Then we have that $\|M f\|_{\mathcal{M}_{q}^{p}(d x, w)} \lesssim p, q, w$ $\|f\|_{\mathcal{M}_{q}^{p}(d x, w)}$ for all $f \in \mathcal{M}_{q}^{p}(d x, w)$.

pr-151201-2

$160121-8$

$160121-9$

Proposition 3.5. Let $0<q \leq p<\infty, \lambda \in\left(0,2^{-1}\right)$ and $w \in A_{\infty}$. Then for $f \in L^{0}\left(\mathbb{R}^{n}\right)$, we have that

$$
\left\|M_{\lambda}^{\sharp, d} f\right\|_{\mathcal{M}_{q}^{p}(w, w)} \lesssim_{\lambda, p, q, w}\|f\|_{\mathcal{M}_{q}^{p}(w, w)} .
$$

Moreover, if we assume that $w \in A_{\infty} \cap \mathcal{B}_{p, q}$, then for $f \in L^{0}\left(\mathbb{R}^{n}\right)$, we have that

$$
\left\|M_{\lambda}^{\sharp, d} f\right\|_{\mathcal{M}_{q}^{p}(d x, w)} \lesssim_{\lambda, p, q, w}\|f\|_{\mathcal{M}_{q}^{p}(d x, w)} .
$$

Proof. First, we observe that for any $\eta>0$,

$$
M_{\lambda}^{\sharp, d} f(x) \lesssim_{\lambda, \eta} M^{(\eta)} f(x) .
$$

To see this, let $Q \in \mathcal{Q}$ and $m_{f}(Q)$ be any median. We see that by (2.11),

$$
\begin{aligned}
\omega_{\lambda}(f ; Q) & \leq\left(\left(f-m_{f}(Q)\right) \chi_{Q}\right)^{*}(\lambda|Q|) \\
& \leq\left(f \cdot \chi_{Q}\right)^{*}\left(\frac{\lambda}{2}|Q|\right)+\left(m_{f}(Q) \chi_{Q}\right)^{*}\left(\frac{\lambda}{2}|Q|\right) \leq 2\left(f \cdot \chi_{Q}\right)^{*}\left(\frac{\lambda}{2}|Q|\right) .
\end{aligned}
$$

Moreover, we notice that

$$
\left(f \cdot \chi_{Q}\right)^{*}\left(\frac{\lambda}{2}|Q|\right) \leq\left(\frac{2}{\lambda|Q|} \int_{0}^{\frac{\lambda}{2}|Q|}\left(f \cdot \chi_{Q}\right)^{*}(t)^{\eta} d t\right)^{\frac{1}{\eta}} \leq\left(\frac{2}{\lambda|Q|} \int_{Q}|f(x)|^{\eta} d x\right)^{\frac{1}{\eta}} .
$$

In summary, we conclude that

$$
\begin{aligned}
M_{\lambda}^{\sharp, d} f(x) & \leq 2 \sup _{Q_{0} \in \mathcal{Q}} \sup _{Q \in \mathcal{D}\left(Q_{0}\right)}\left(f \cdot \chi_{Q}\right)^{*}\left(\frac{\lambda}{2}|Q|\right) \chi_{Q}(x) \\
& \leq 2^{1+\frac{1}{\eta}} \lambda^{-\frac{1}{\eta}} \sup _{Q_{0} \in \mathcal{Q}} \sup _{Q \in \mathcal{D}\left(Q_{0}\right)}\left(\frac{1}{|Q|} \int_{Q}|f(x)|^{\eta} d x\right)^{\frac{1}{\eta}} \chi_{Q}(x),
\end{aligned}
$$

which implies (3.3). Next, we choose $u \in(1, \infty)$ such that $w \in A_{u}$ and $\eta:=\frac{q}{u}$. In view of (3.3) and Theorem 3.4, it follows that

$$
\left\|M_{\lambda}^{\sharp, d} f\right\|_{\mathcal{M}_{q}^{p}(w, w)} \lesssim_{\lambda, \eta}\left\|M^{(\eta)} f\right\|_{\mathcal{M}_{q}^{p}(w, w)} \lesssim_{p, q, w}\|f\|_{\mathcal{M}_{q}^{p}(w, w)},
$$

which proves (3.1).

Meanwhile, if we observe that $w \in \mathcal{B}_{p, q}$ is equivalent to $w \in \mathcal{B}_{\frac{p}{n}, \frac{q}{\eta}}$; see [?], then by (3.3) and Theorem 3.4, it follows that

$$
\left\|M_{\lambda}^{\sharp, d} f\right\|_{\mathcal{M}_{q}^{p}(d x, w)} \lesssim_{\lambda, \eta}\left\|M^{(\eta)} f\right\|_{\mathcal{M}_{q}^{p}(d x, w)} \lesssim_{p, q, w}\|f\|_{\mathcal{M}_{q}^{p}(d x, w)},
$$


which proves (3.2).

By combining (1.16), (1.17), Corollary 3.3 and Proposition 3.5, we obtain Theorem 1.11 Next, let us prove Theorem 1.12. We will employ a similar method to the proof of Theorem 3.2

Proof of Theorem 1.12. First, let us show that

$$
\|f\|_{\mathcal{M}_{s}^{p}(w, w)} \lesssim_{p, s, w}\left\|M_{\lambda_{w}}^{\sharp, d} f\right\|_{\mathcal{M}_{s}^{p}(w, w)}
$$

by assuming $\lim _{l \rightarrow \infty} m_{f}\left(2^{l} Q_{0}\right) \rightarrow 0$ for all $Q_{0} \in \mathcal{Q}$. To this end, we fix any $Q_{0} \in \mathcal{Q}$ and calculate that

$$
\begin{gathered}
w\left(Q_{0}\right)^{\frac{1}{p}-\frac{1}{s}}\left(\int_{Q_{0}}|f(x)|^{s} d w(x)\right)^{\frac{1}{s}} \lesssim_{s} w\left(Q_{0}\right)^{\frac{1}{p}-\frac{1}{s}}\left(\int_{Q_{0}}\left|f(x)-m_{f}\left(2^{l} Q_{0}\right)\right|^{s} d w(x)\right)^{\frac{1}{s}} \\
+w\left(Q_{0}\right)^{\frac{1}{p}}\left|m_{f}\left(2^{l} Q_{0}\right)\right| .
\end{gathered}
$$

By the assumption, it follows that

$$
w\left(Q_{0}\right)^{\frac{1}{p}-\frac{1}{s}}\left(\int_{Q_{0}}|f(x)|^{s} d w(x)\right)^{\frac{1}{s}} \lesssim_{s} \limsup _{l \rightarrow \infty} w\left(Q_{0}\right)^{\frac{1}{p}-\frac{1}{s}}\left(\int_{Q_{0}}\left|f(x)-m_{f}\left(2^{l} Q_{0}\right)\right|^{s} d w(x)\right)^{\frac{1}{s}},
$$

and hence, we focus on the quantity: $\left(\int_{Q_{0}}\left|f(x)-m_{f}\left(2^{l} Q_{0}\right)\right|^{s} d w(x)\right)^{\frac{1}{s}}$. We employ Proposition 3.1 again to decompose $f-m_{f}\left(2^{l} Q_{0}\right)$ and obtain that

$$
\left(\int_{Q_{0}}\left|f(x)-m_{f}\left(2^{l} Q_{0}\right)\right|^{s} d w(x)\right)^{\frac{1}{s}} \lesssim s\left(\int_{Q_{0}} M_{\lambda_{w}}^{\sharp, d} f(x)^{s} d w(x)\right)^{\frac{1}{s}}+\mathrm{I},
$$

where we defined

$$
\mathrm{I}:=\left[\int_{Q_{0}}\left(\sum_{k \in \mathbb{N}_{0}} \sum_{j \in J_{k}} \omega_{\lambda_{w}}\left(f ; Q_{j}^{k}\right) \chi_{Q_{j}^{k}}(x)\right)^{s} d w(x)\right]^{\frac{1}{s}} .
$$

Here, we remark that the family $\left\{Q_{j}^{k}\right\}_{k \in \mathbb{N}_{0}, j \in J_{k}} \subset \mathcal{D}\left(2^{l} Q_{0}\right)$ is a $w$-sparse family generated by $2^{l} Q_{0}$. To evaluate I, by considering the suitable dyadic setting, we have only to calculate the following two terms intrinsically:

$$
\begin{aligned}
\mathrm{I}_{a} & :=\left[\int_{Q_{0}}\left(\sum_{k \in \mathbb{N}_{0}} \sum_{\substack{j \in J_{k}: \\
Q_{j}^{k} \subset Q_{0}}} \omega_{\lambda_{w}}\left(f ; Q_{j}^{k}\right) \chi_{Q_{j}^{k}}(x)\right)^{s} d w(x)\right]^{\frac{1}{s}}, \\
\mathrm{I}_{b} & :=\left[\int_{Q_{0}}\left(\sum_{k \in \mathbb{N}_{0}} \sum_{\substack{j \in J_{k}: \\
Q_{j}^{k} \supsetneq Q_{0}}} \omega_{\lambda_{w}}\left(f ; Q_{j}^{k}\right) \chi_{Q_{j}^{k}}(x)\right]^{\frac{1}{s}} d w(x)\right]^{s} .
\end{aligned}
$$

For $\mathrm{I}_{a}$, we employ the same method used in the proof of Theorem 3.2, namely, by observing $\chi_{Q_{j}^{k}} \leq C_{w} M_{w}\left[\chi_{Q_{j}^{k} \cap \Omega_{k+1}^{c}}\right]$ and using the boundedness of $M_{w}$, it follows that

$$
\mathrm{I}_{a} \lesssim_{s, w}\left[\int_{Q_{0}}\left(\sum_{k \in \mathbb{N}_{0}} \sum_{\substack{j \in J_{k}: \\ Q_{j}^{k} \subset Q_{0}}} \omega_{\lambda_{w}}\left(f ; Q_{j}^{k}\right) \chi_{Q_{j}^{k} \cap \Omega_{k+1}^{c}}(x)\right)^{s} d w(x)\right]^{\frac{1}{s}} .
$$


If we notice that the summation is taken over the cubes contained in $Q_{0}$, then the disjointness of $\left\{Q_{j}^{k} \cap \Omega_{k+1}^{c}\right\}_{k \in \mathbb{N}_{0}, j \in J_{k}}$ yields that

$$
\mathrm{I}_{a} \lesssim s, w\left(\int_{Q_{0}} M_{\lambda_{w}}^{\sharp, d} f(x)^{s} d w(x)\right)^{\frac{1}{s}}
$$

Meanwhile, for $\mathrm{I}_{b}$, by recalling that $Q_{j}^{k} \subset 2^{l} Q_{0}$ and the dyadic property, we can rewrite the summation of $\mathrm{I}_{b}$ as follows:

$$
\mathrm{I}_{b} \leq\left[\int_{Q_{0}}\left(\sum_{m=1}^{l} \omega_{\lambda_{w}}\left(f ; Q_{0}^{(m)}\right) \chi_{Q_{0}^{(m)}}(x)\right)^{s} d w(x)\right]^{\frac{1}{s}} .
$$

Here, $Q_{0}^{(m)}$ denotes the dyadic $m$-th ancestor of $Q_{0}$, that is, $Q_{0}^{(m)}$ is a unique dyadic cube with respect to $2^{l} Q_{0}$ whose side length is $2^{m} \ell\left(Q_{0}\right)$ and containing $Q_{0}$. With the relation: $Q_{0} \subset Q_{0}^{(m)}$ in mind, we see that

$$
\begin{aligned}
\mathrm{I}_{b} & \leq w\left(Q_{0}\right)^{\frac{1}{s}} \sum_{m=1}^{l} \omega_{\lambda_{w}}\left(f ; Q_{0}^{(m)}\right) \\
& =w\left(Q_{0}\right)^{\frac{1}{s}} \sum_{m=1}^{l} \frac{1}{w\left(Q_{0}^{(m)}\right)^{\frac{1}{s}}}\left(\int_{Q_{0}^{(m)}} \omega_{\lambda_{w}}\left(f ; Q_{0}^{(m)}\right)^{s} d w(x)\right)^{\frac{1}{s}} \\
& \leq w\left(Q_{0}\right)^{\frac{1}{s}} \sum_{m=1}^{l} \frac{1}{w\left(Q_{0}^{(m)}\right)^{\frac{1}{p}}}\left\|M_{\lambda_{w}}^{\sharp, d} f\right\|_{\mathcal{M}_{s}^{p}(w, w)} .
\end{aligned}
$$

Now, let us show that

$$
\sum_{m=1}^{l} \frac{1}{w\left(Q_{0}^{(m)}\right)^{\frac{1}{p}}} \lesssim_{p, w} \frac{1}{w\left(Q_{0}\right)^{\frac{1}{p}}} .
$$

Since $w \in A_{\infty}$, there exist $L_{w} \in \mathbb{N}$ and $\alpha_{w}>1$ such that

$$
w\left(Q_{0}^{\left(i+L_{w}\right)}\right) \geq \alpha_{w} w\left(Q_{0}^{(i)}\right), \quad\left(i \in \mathbb{N}_{0}, Q_{0} \in \mathcal{D}\right) .
$$

In particular, for $i, j \in \mathbb{N}$, it holds that

$$
w\left(Q_{0}^{\left(i+j L_{w}\right)}\right) \geq \alpha_{w} w\left(Q_{0}^{\left(i+(j-1) L_{w}\right)}\right) \geq \cdots \geq \alpha_{w}^{j} w\left(Q_{0}^{(i)}\right) .
$$

Hence, the left-hand side of (3.8) can be controlled as follows:

$$
\sum_{m=1}^{l} \frac{1}{w\left(Q_{0}^{(m)}\right)^{\frac{1}{p}}} \leq \sum_{i=1}^{L_{w}} \sum_{j=0}^{\left[l / L_{w}\right]+1} \frac{1}{w\left(Q_{0}^{\left(i+j L_{w}\right)}\right)^{\frac{1}{p}}} \leq \sum_{i=1}^{L_{w}} \frac{1}{w\left(Q_{0}^{(i)}\right)^{\frac{1}{p}}} \sum_{j=0}^{\left[l / L_{w}\right]+1} \frac{1}{\alpha_{w}^{\frac{j}{p}}} .
$$

If we recall that $w\left(Q_{0}\right) \leq w\left(Q_{0}^{(i)}\right)$ for any $i \in \mathbb{N}$ and that $\alpha_{w}>1$, then we see that

$$
\sum_{m=1}^{l} \frac{1}{w\left(Q_{0}^{(m)}\right)^{\frac{1}{p}}} \lesssim_{p, w} \frac{L_{w}}{w\left(Q_{0}\right)^{\frac{1}{p}}},
$$

which implies (3.8), since the constant $L_{w}$ and $\alpha_{w}$ depend only on $w$ (and dimension $n$ ). Remark that the implicit constant in (3.8) does not depend on $l \in \mathbb{N}$ as well. Therefore, we obtain the estimate of $\mathrm{I}_{b}$ :

$$
\mathrm{I}_{b} \lesssim_{p, w} w\left(Q_{0}\right)^{-\frac{1}{p}+\frac{1}{s}}\left\|M_{\lambda_{w}}^{\sharp, d} f\right\|_{\mathcal{M}_{s}^{p}(w, w)} .
$$


In total, it follows from (3.6), (3.7) and (3.10) that

$$
\begin{aligned}
\left(\int_{Q_{0}}\left|f(x)-m_{f}\left(2^{l} Q_{0}\right)\right|^{s} d w(x)\right)^{\frac{1}{s}} \lesssim_{p, s, w} & \left(\int_{Q_{0}} M_{\lambda_{w}}^{\sharp, d} f(x)^{s} d w(x)\right)^{\frac{1}{s}} \\
& +w\left(Q_{0}\right)^{-\frac{1}{p}+\frac{1}{s}}\left\|M_{\lambda_{w}}^{\sharp, d} f\right\|_{\mathcal{M}_{s}^{p}(w, w)}
\end{aligned}
$$

and hence, in view of (3.5), we conclude (3.4).

Next, assuming the weighted integral condition (1.3) for $p, q$ and $w$, we prove

$$
\|f\|_{\mathcal{M}_{q}^{p}(d x, w)} \lesssim_{p, q, w}\left\|M_{\lambda_{w}^{\sharp, d}}^{\sharp}\right\|_{\mathcal{M}_{q}^{p}(d x, w)} .
$$

Another inequality:

$$
\sup _{Q \in \mathcal{Q}} \Phi_{p, q, w}(Q)\left(\frac{1}{w(Q)} \int_{Q}|f(x)|^{s} d w(x)\right)^{\frac{1}{s}} \leq\|f\|_{\mathcal{M}_{q}^{p}(d x, w)}
$$

follows from Hölder's inequality directly. We need only mimic the above proof. Fix any $Q_{0} \in \mathcal{D}$ and calculate that

$$
\left|Q_{0}\right|^{\frac{1}{p}-\frac{1}{q}}\left(\int_{Q_{0}}|f(x)|^{q} d w(x)\right)^{\frac{1}{q}} \lesssim_{q} \limsup _{l \rightarrow \infty}\left|Q_{0}\right|^{\frac{1}{p}-\frac{1}{q}}\left(\int_{Q_{0}}\left|f(x)-m_{f}\left(2^{l} Q_{0}\right)\right|^{q} d w(x)\right)^{\frac{1}{q}} .
$$

Again, Proposition 3.1. Lerner's decomposition formula reduces the matters to show the inequality: $\mathrm{II}_{a}+\mathrm{II}_{b} \lesssim_{p, q, w}\left\|M_{\lambda_{w}}^{\sharp, d} f\right\|_{\mathcal{M}_{q}^{p}(d x, w)}$, where,

$$
\begin{aligned}
& \mathrm{II}_{a}:=\left|Q_{0}\right|^{\frac{1}{p}-\frac{1}{q}}\left[\int_{Q_{0}}\left(\sum_{\substack{k \in \mathbb{N}_{0} \\
Q_{j} \subset Q_{0}}} \sum_{\substack{j \in J_{k}: \\
Q_{j}^{k}}} \omega_{\lambda_{w}}\left(f ; Q_{j}^{k}\right) \chi_{Q_{j}^{k}}(x)\right]^{\frac{1}{s}} d w(x)\right]^{s}
\end{aligned}
$$

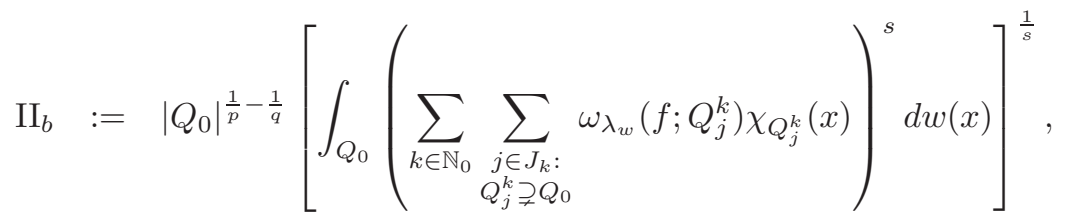

and $\left\{Q_{j}^{k}\right\}_{k \in \mathbb{N}_{0}, j \in J_{k}} \subset \mathcal{D}\left(2^{l} Q_{0}\right)$ is a $w$-sparse family generated by $2^{l} Q_{0}$. For $\mathrm{II}_{a}$, we may apply the same argument used to evaluate $I_{a}$; see (3.7) to obtain that

$$
\mathrm{II}_{a} \lesssim q, w\left|Q_{0}\right|^{\frac{1}{p}-\frac{1}{q}}\left(\int_{Q_{0}} M_{\lambda_{w}}^{\sharp, d} f(x)^{q} d w(x)\right)^{\frac{1}{q}} \leq\left\|M_{\lambda_{w}}^{\sharp, d} f\right\|_{\mathcal{M}_{q}^{p}(d x, w)} .
$$

For $\mathrm{II}_{b}$, we employ the weighted integral condition (1.3) for $p, q$ and $w$ instead of the $A_{\infty}$ condition. As before, using the dyadic $m$-ancestor of $Q_{0}$, we see that

$$
\begin{aligned}
\mathrm{II}_{b} & \leq\left|Q_{0}\right|^{\frac{1}{p}-\frac{1}{q}}\left(\int_{Q_{0}}\left(\sum_{m=1}^{l} \omega_{\lambda_{w}}\left(f ; Q_{0}^{(m)}\right) \chi_{Q_{0}^{(m)}}(x)\right)^{q} d w(x)\right)^{\frac{1}{q}} \\
& =\Phi_{p, q, w}\left(Q_{0}\right) \sum_{m=1}^{l} \omega_{\lambda_{w}}\left(f ; Q_{0}^{(m)}\right) \\
& \leq \Phi_{p, q, w}\left(Q_{0}\right) \sum_{m=1}^{l} \frac{1}{w\left(Q_{0}^{(m)}\right)^{\frac{1}{q}}}\left(\int_{Q_{0}^{(m)}} M_{\lambda_{w}^{\sharp, d}}^{\sharp} f(x)^{q} d w(x)\right)^{\frac{1}{q}} .
\end{aligned}
$$


From the definition of the norm of $\mathcal{M}_{q}^{p}(d x, w)$, and the weighted integral condition (1.3) it follows that

$$
\mathrm{II}_{b} \leq \Phi_{p, q, w}\left(Q_{0}\right) \sum_{m=1}^{l} \frac{1}{\Phi_{p, q, w}\left(Q_{0}^{(m)}\right)}\left\|M_{\lambda}^{\sharp, d} f\right\|_{\mathcal{M}_{q}^{p}(d x, w)} \lesssim_{p, q, w}\left\|M_{\lambda}^{\sharp, d} f\right\|_{\mathcal{M}_{q}^{p}(d x, w)} .
$$

By invoking Lemma 2.7 in Chapter 2 and (1.14), we obtain the direct analogy from original type of the sharp maximal inequality :

Corollary 3.6. Let $0<q \leq p<\infty$ and $w \in A_{\infty}$.

(1) (Komori-Shirai type) For any $f \in L^{0}\left(\mathbb{R}^{n}\right)$ satisfying $M f \in \mathcal{M}_{q_{0}}^{p_{0}}\left(w_{0}, w_{0}\right)$ for some $0<q_{0} \leq p_{0}<\infty$ and $w_{0} \in A_{\infty}$, we have that

$$
\|f\|_{\mathcal{M}_{q}^{p}(w, w)} \sim_{p, q, w}\left\|M_{\lambda_{w}}^{\sharp, d} f\right\|_{\mathcal{M}_{q}^{p}(w, w)} \lesssim \lambda_{w}\left\|f^{\sharp}\right\|_{\mathcal{M}_{q}^{p}(w, w)} .
$$

(2) (Samko type) For any $f \in L^{0}\left(\mathbb{R}^{n}\right)$ satisfying $M f \in \mathcal{M}_{q_{0}}^{p_{0}}\left(d x, w_{0}\right)$ for some $0<q_{0} \leq$ $p_{0}<\infty$ and $w_{0}$ satisfying the weighted integral condition (1.3) for $p_{0}, q_{0}$ and $w_{0}$, we have that

$$
\|f\|_{\mathcal{M}_{q}^{p}(d x, w)} \sim_{p, q, w}\left\|M_{\lambda_{w}}^{\sharp, d} f\right\|_{\mathcal{M}_{q}^{p}(d x, w)} \lesssim \lambda_{w}\left\|f^{\sharp}\right\|_{\mathcal{M}_{q}^{p}(d x, w)} .
$$

Let us compare the above results with some recent researches. Sawano and Tanaka in 26] proved the following refinement of sharp maximal inequality:

Theorem 3.7. 26, Theorem 1.3] Let $1<q \leq p<\infty$. Then for any $f \in L^{0}\left(\mathbb{R}^{n}\right)$, we have that

$$
\|M f\|_{\mathcal{M}_{q}^{p}(d x, d x)} \lesssim_{p, q}\left\|f^{\sharp}\right\|_{\mathcal{M}_{q}^{p}(d x, d x)}+\|f\|_{\mathcal{M}_{1}^{p}(d x, d x)} .
$$

In view of (1.14) and Theorem 3.4 Theorem 1.11 improves and generalizes Theorem 3.7 Meanwhile, there exists a weighted result for the sharp maximal inequality obtained by KomoriFuruya in [14:

Theorem 3.8. 14, Theorem 12] Let $1<q_{0} \leq q \leq p<\infty$ and $w \in A_{\infty}$. Then for $f \in L^{0}\left(\mathbb{R}^{n}\right)$ satisfying $M f \in \mathcal{M}_{q_{0}}^{p}(w, w)$, it holds that

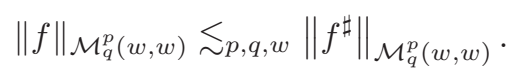

We can see easily that Corollaries 1.14 and 3.6 improve and generalize Theorem 3.8

\section{Proof of Theorems 1.3 and 1.8 including Another application}

We will show Theorem 1.3 with more general settings. Recall the definition of the singular integral operators. A singular integral operator $T$ is a bounded linear operator on the unweighted $L^{2}$ space, for which there exists a kernel $K$ on $\mathbb{R}^{n} \times \mathbb{R}^{n}$ satisfying the following conditions:

(1) (Size condition) There exists $C>0$ such that $|K(x, y)| \leq \frac{C}{|x-y|^{n}}$ for any $x \neq y$.

(2) (Hörmander condition) For some $\theta \in(0,1]$,

$$
|K(x+h, y)-K(x, y)|+|K(x, y+h)-K(x, y)| \leq \frac{C|h|^{\theta}}{|x-y|^{n+\theta}}, \quad \text { if }|x-y|>2|h| .
$$


(3) If $f \in L_{\mathrm{c}}^{\infty}$, the set of all compactly supported $L^{\infty}$-functions, then

$$
T f(x)=\int_{\mathbb{R}^{n}} K(x, y) f(y) d y \quad(x \notin \operatorname{supp}(f)) .
$$

As Coifman and Fefferman showed in [4, Theorems I and III], $T$ is well defined as a bounded operator on $L^{q}(w)$, when $w \in A_{q}$ with $1<q<\infty$.

We need to recall some examples of the singular integral operators.

Definition 4.1 (3]). A singular integral operator $T$ is called a genuine singular integral operator if there exist constants $C, \theta>0$ and $R \in O(n)$ such that for any $x, y \in \mathbb{R}^{n}$ satisfying $x-y \in V_{\theta, R}:=R\left\{u=\left(u^{\prime}, u_{n}\right) \in \mathbb{R}^{n}:\left|u^{\prime}\right|<\theta\left|u_{n}\right|\right\}$, the integral kernel $K$ satisfies

$$
K(x, y) \geq \frac{C}{|x-y|^{n}},
$$

where $O(n)$ denotes the set of all orthogonal matrices in $\mathbb{R}^{n}$.

A typical example of the genuine singular integral operators is the Riesz transform. Our aim in this section is to show the following theorem:

Theorem 4.2. Let $1<q \leq p<\infty$ and $w$ be a weight.

(1) Assume that $M$ is bounded on $\mathcal{M}_{q}^{p}(d x, w)$ and the weighted integral condition (1.3) for $p, q$ and $w$. Then we can extend any singular integral operator $T_{0}$ initially defined on $L_{\mathrm{c}}^{\infty}$ to a bounded linear operator $T$ on $\mathcal{M}_{q}^{p}(d x, w)$ :

$$
\|T f\|_{\mathcal{M}_{q}^{p}(d x, w)} \leq C\|f\|_{\mathcal{M}_{q}^{p}(d x, w)} \quad\left(f \in \mathcal{M}_{q}^{p}(d x, w)\right) .
$$

(2) As a converse assertion, we have the following:

(a) If the Riesz transform $R_{i}$ is bounded on $\mathcal{M}_{q}^{p}(d x, w)$ for some $i=1, \ldots, n$, then $w$ satisfies (1.7).

(b) Assume that $w \in \mathcal{B}_{p, q}$ satisfies the doubling condition. If there exists a genuine singular integral operator $T$ which is bounded on $\mathcal{M}_{q}^{p}(d x, w)$, then the weighted integral condition (1.3) for $p, q$ and $w$ holds.

Once we prove Theorem 4.2 then we obtain Theorem 1.3 as a corollary. To see this, we admit Theorem 4.2 for a moment. If the Riesz transform $R_{i}$ is bounded on $\mathcal{M}_{q}^{p}(d x, w)$, then (1.7) holds by the assertion (2)-(a) in Theorem 4.2. Then by Lemma 2.2 or Remark 2.3, we see that $w \in \mathcal{B}_{p, q} \cap A_{q+1}$, particularly, $w$ is doubling. Here, we recall that the Riesz transform $R_{i}$ is an example of the genuin singular integral operators. Hence, we may apply the assertion (2)-(b) in Theorem (4.2) to obtain the weighted integral condition (1.3) for $p, q$ and $w$. This means the assertion (2) in Theorem 1.3 The assertion (1) in Theorem 1.3 is clear from the assertion (1) in Theorem 4.2

Since the proof of the assertion (1) of Theorem 4.2 is easier than the one of Theorem 1.8 , we prove only the assertion (2) of Theorem 4.2 here.

Proof of Theorem 4.2. (2)-(a). Assume that $R_{i}$ is bounded on $\mathcal{M}_{q}^{p}(d x, w)$ for some $i=1, \ldots, n$. To show (1.7), we fix any $Q \in \mathcal{Q}$ and take any $f \geq 0$ such that $f \cdot \chi_{Q} \in \mathcal{M}_{q}^{p}(d x, w)$. We focus on the cube $\widetilde{Q}:=Q+2 \ell(Q) e_{i}$. Here, $e_{i}$ denotes the $i$-th elementary vector. If we notice that for any $x \in \widetilde{Q}$, it holds that $x_{i}-y_{i} \geq \ell(Q)$ and that $|x-y| \leq C \ell(Q)$, then we have the pointwise estimate

$$
\left|R_{i}\left[f \cdot \chi_{Q}\right](x)\right|=\int_{Q} f(y) \frac{x_{i}-y_{i}}{|x-y|^{n+1}} d y \geq C \frac{1}{|Q|} \int_{Q} f(y) d y .
$$


Hence it follows from the boundedness of $R_{i}$ that

$$
\frac{1}{|Q|} \int_{Q} f(y) d y \leq \frac{C}{\left\|\chi_{\widetilde{Q}}\right\|_{\mathcal{M}_{q}^{p}(d x, w)}}\left\|R_{i}\left[f \cdot \chi_{Q}\right] \chi_{\widetilde{Q}}\right\|_{\mathcal{M}_{q}^{p}(d x, w)} \leq C \frac{\left\|f \cdot \chi_{Q}\right\|_{\mathcal{M}_{q}^{p}(d x, w)}}{\left\|\chi_{\widetilde{Q}}\right\|_{\mathcal{M}_{q}^{p}(d x, w)}}
$$

Namely, we have that for any $f \geq 0$ such that $f \cdot \chi_{Q} \in \mathcal{M}_{q}^{p}(d x, w)$,

160114-7

160114-8

160114-9

160114-10

$$
\frac{1}{|Q|} \int_{Q} f(y) d y \cdot\left\|\chi_{\widetilde{Q}}\right\|_{\mathcal{M}_{q}^{p}(d x, w)} \leq C\left\|f \cdot \chi_{Q}\right\|_{\mathcal{M}_{q}^{p}(d x, w)}
$$

Now, we take any $g \geq 0$ such that $\left\|g \cdot \chi_{\widetilde{Q}}\right\|_{\mathcal{M}_{q}^{p}(d x, w)} \leq 1$ and put $f=\left|R_{i}\left[g \cdot \chi_{\widetilde{Q}}\right]\right|$ as in (4.1) to obtain that

$$
\frac{1}{|Q|} \int_{Q}\left|R_{i}\left[g \cdot \chi_{\widetilde{Q}}\right](x)\right| d x \cdot\left\|\chi_{\widetilde{Q}}\right\|_{\mathcal{M}_{q}^{p}(d x, w)} \leq C\left\|g \cdot \chi_{\widetilde{Q}}\right\|_{\mathcal{M}_{q}^{p}(d x, w)} \leq C .
$$

Here, we again used the boundedness of $R_{i}$. Meanwhile, if we go through a similar argument as before, we notice

$$
\frac{1}{|\widetilde{Q}|} \int_{\widetilde{Q}} g(y) d y \leq C \inf _{x \in Q}\left|R_{i}\left[g \cdot \chi_{\widetilde{Q}}\right](x)\right| \leq C \frac{1}{|Q|} \int_{Q}\left|R_{i}\left[g \cdot \chi_{\widetilde{Q}}\right](x)\right| d x .
$$

By combining (4.2) and (4.3), it follows that for any $g \geq 0$ such that $\left\|g \cdot \chi_{\widetilde{Q}}\right\|_{\mathcal{M}_{q}^{p}(d x, w)} \leq 1$,

$$
\frac{1}{|\widetilde{Q}|} \int_{\widetilde{Q}} g(y) d y \cdot\left\|\chi_{\widetilde{Q}}\right\|_{\mathcal{M}_{q}^{p}(d x, w)} \leq C
$$

Moreover using (1.6), we see that

$$
\frac{1}{|\widetilde{Q}|}\left\|\chi_{\widetilde{Q}}\right\|_{\mathcal{M}_{q}^{p}(d x, w)}\left\|w^{-\frac{1}{q}} \chi_{\widetilde{Q}}\right\|_{H^{q^{\prime}, n(1-q / p)}}=\frac{\left\|\chi_{\widetilde{Q}}\right\|_{\mathcal{M}_{q}^{p}(d x, w)}}{|\widetilde{Q}|} \sup _{\left\|g \chi_{\widetilde{Q}}\right\|_{\mathcal{M}_{q}^{p}(d x, w)} \leq 1} \int_{\widetilde{Q}} g(y) d y \leq C,
$$

which implies (1.7).

Next, we prove the assertion (2)-(b) in Theorem 4.2. That is, we show the weighted integral condition (1.3) for $p, q$ and $w$ by assuming that $w \in \mathcal{B}_{p, q}$ satisfies the doubling condition and that a certain genuine singular integral operator $T$ is bounded on $\mathcal{M}_{q}^{p}(d x, w)$. To this end, we recall the equivalent condition of the weighted integral condition (1.3).

lm-151222-1 Lemma 4.3 ([22]). Let $0<q \leq p<\infty$ and $w \in \mathcal{B}_{p, q}$ satisfies the doubling condition. Then the following are equivalent:

(1) The weighted integral condition for $p, q$ and $w$ holds.

(2) There exists $c>1$ such that

$$
2 \Phi_{p, q, w}(Q) \leq \Phi_{p, q, w}(c Q)
$$

for any $Q \in \mathcal{Q}$.

The idea of the following proof goes back to [8, Theorem 6.9].

Proof of Theorem 4.2-(2)-(b). By assuming that the weighted integral condition (1.3) for $p, q$ and $w$ fails to hold, let us obtain the contradiction. With Lemma 4.3 in mind, we assume that for any $m \in \mathbb{N}$, there exists $Q_{m} \in \mathcal{Q}$ such that $2 \Phi_{p, q, w}\left(Q_{m}\right)>\Phi_{p, q, w}\left(2^{m} Q_{m}\right)$. We take a genuine singular integral operator $T$ and denote the cone $V_{\theta, R}$ by $V$. Set

$$
f_{m}(y):=\chi_{V-c\left(Q_{m}\right)}(-y) \chi_{2^{m-1} Q_{m} \backslash 2^{6} Q_{m}}(y),
$$

for $m \geq 7$. Since $V$ is a cone, we may take another cube $R_{m}$ so that $R_{m} \subset\left(V+c\left(Q_{m}\right)\right) \cap$ $\left(2^{4} Q_{m} \backslash Q_{m}\right), Q_{m} \subset C_{V} R_{m}$ for some $C_{V}>0$ and that $\left|R_{m}\right| \sim_{V}\left|Q_{m}\right|$. Note that for any 
$x \in R_{m}$ and any $y \in 2^{m-1} Q_{m} \backslash 2{ }^{6} Q_{m}$ such that $-y \in V-c\left(Q_{m}\right)$, we have $x-y \in V$ and $\ell\left(Q_{m}\right) \lesssim|x-y| \lesssim 2^{m} \ell\left(Q_{m}\right)$. Hence, it follows that for any $x \in R_{m}$,

$$
\begin{aligned}
T f_{m}(x) & =\int_{\mathbb{R}^{n}} K(x, y) \chi_{V-c\left(Q_{m}\right)}(-y) \chi_{2^{m-1} Q_{m} \backslash 2^{6} Q_{m}}(y) d y \\
& \sim \int_{\left\{x-y \in V: \ell\left(Q_{m}\right)<|x-y| \leq 2^{m} \ell\left(Q_{m}\right)\right\}} K(x, y) f_{m}(y) d y \\
& \gtrsim \int_{\left\{\ell\left(Q_{m}\right)<|x-y| \leq 2^{m} \ell\left(Q_{m}\right)\right\}} \frac{d y}{|x-y|^{n}} \\
& \sim m .
\end{aligned}
$$

This implies that $m \cdot \chi_{R_{m}}(x) \lesssim T f_{m}(x)$. Meanwhile, if we recall that $Q_{m} \subset C_{V} R_{m},\left|Q_{m}\right| \sim\left|R_{m}\right|$ and $w$ is a doubling weight, then we see that

$$
\Phi_{p, q, w}\left(Q_{m}\right) \lesssim\left|R_{m}\right|^{\frac{1}{p}}\left(\frac{w\left(C_{V} R_{m}\right)}{\left|R_{m}\right|}\right)^{\frac{1}{q}} \lesssim \Phi_{p, q, w}\left(R_{m}\right) \leq\left\|\chi_{R_{m}}\right\|_{\mathcal{M}_{q}^{p}(d x, w)} .
$$

Hence, the boundedness of $T$ yields

$$
\Phi_{p, q, w}\left(Q_{m}\right) \lesssim \frac{1}{m}\left\|T f_{m}\right\|_{\mathcal{M}_{q}^{p}(d x, w)} \lesssim \frac{1}{m}\left\|\chi_{2^{m} Q_{m}}\right\|_{\mathcal{M}_{q}^{p}(d x, w)} .
$$

Moreover, by $w \in \mathcal{B}_{p, q}$ and $\Phi_{p, q, w}\left(2^{m} Q_{m}\right)<2 \Phi_{p, q, w}\left(Q_{m}\right)$, we see that

$$
\Phi_{p, q, w}\left(Q_{m}\right) \lesssim \frac{1}{m} \Phi_{p, q, w}\left(2^{m} Q_{m}\right) \leq \frac{2}{m} \Phi_{p, q, w}\left(Q_{m}\right),
$$

which implies $1 \lesssim m^{-1}$ holds for any $m \in \mathbb{N}$. This is a contradiction.

Next, we turn into the proof of Theorem 1.8 Again, we prove Theorem 1.8 with more general setting as follows.

th-160626-2 Theorem 4.4. Let $1<q \leq p<\infty, b \in \mathrm{BMO}$ and $T$ be a singular integral operator. Assume that $M$ is bounded on $\mathcal{M}_{q}^{p}(d x, w)$ and the weighted integral condition (1.3) for $p, q$ and $w$. Then we can extend the commutator $[b, T]$ as a bounded linear operator on $\mathcal{M}_{q}^{p}(d x, w)$ :

$$
\|[b, T] f\|_{\mathcal{M}_{q}^{p}(d x, w)} \leq C\|f\|_{\mathcal{M}_{q}^{p}(d x, w)} \quad\left(f \in \mathcal{M}_{q}^{p}(d x, w)\right) .
$$

To show Theorem [4.4, we first extend the definition of the commutator $[b, T]$ as a linear operator defined on $\mathcal{M}_{q}^{p}(d x, w)$. We remark that once we assume the boundedness of $M$ on $\mathcal{M}_{q}^{p}(d x, w)$, then $w \in A_{\infty}$ automatically by Lemma 2.2 Let $b \in \mathrm{BMO}$ and $T$ be a singular integral operator. Moreover, we take $\varepsilon \in(0,1)$ as in Corollary 2.10. For $f \in \mathcal{M}_{q}^{p}(d x, w)$ and $x \in \mathbb{R}^{n}$, we define

$$
[b, T] f(x):=[b, T]_{0}\left(f \cdot \chi_{2 Q}\right)(x)+\int_{\mathbb{R}^{n} \backslash 2 Q}(b(x)-b(y)) K(x, y) f(y) d y,
$$

where $Q$ is any cube containing the point $x$ and $[b, T]_{0}$ denotes the commutator as a bounded linear operator on $L^{\frac{1}{1-\varepsilon}}\left(\mathbb{R}^{n}\right)$. It is easy to check that the definition of $[b, T] f(x)$ does not depend on the choice of the cube $Q$ which contains $x$. In addition, since we know that $f \cdot \chi_{Q} \in L^{\frac{1}{1-\varepsilon}}\left(\mathbb{R}^{n}\right)$ for any $f \in \mathcal{M}_{q}^{p}(d x, w)$ and $Q \in \mathcal{Q}$ by Corollary 2.10 , the first term: $[b, T]_{0}\left(f \cdot \chi_{2 Q}\right)(x)$ is well defined. For the second term, we have the following lemma:

lm-160114-9 Lemma 4.5. Let $1<q \leq p<\infty$ and assume the boundedness of $M$ on $\mathcal{M}_{q}^{p}(d x, w)$ and the weighted integral condition (1.3) for $p, q$ and $w$. Then for any $x \in \mathbb{R}^{n}$ and any $Q \in \mathcal{Q}$ containing $x$, we have that

$$
\int_{\mathbb{R}^{n} \backslash 2 Q}|(b(x)-b(y)) K(x, y) f(y)| d y \leq C \frac{\|f\|_{\mathcal{M}_{q}^{p}(d x, w)}}{\Phi_{p, q, w}(Q)}\left(\|b\|_{\mathrm{BMO}}+\left|b(x)-b_{Q}\right|\right),
$$


where $b_{Q}$ denotes the mean value of $b$ on $Q$ with respect to the Lebesgue measure, that is, $b_{Q}:=\frac{1}{|Q|} \int_{Q} b(x) d x$.

Proof. It follows from the size condition of the kernel $K$ that

$$
\int_{\mathbb{R}^{n} \backslash 2 Q}|(b(x)-b(y)) K(x, y) f(y)| d y \leq C(\mathrm{I}+\mathrm{II}),
$$

where we denined

$$
\mathrm{I}:=\int_{\mathbb{R}^{n} \backslash 2 Q} \frac{\left|b(x)-b_{Q}\right|}{|x-y|^{n}}|f(y)| d y, \quad \mathrm{II}:=\int_{\mathbb{R}^{n} \backslash 2 Q} \frac{\left|b(y)-b_{Q}\right|}{|x-y|^{n}}|f(y)| d y .
$$

We first evaluate the second term II. Since we assume the boundedness of $M$ on $\mathcal{M}_{q}^{p}(d x, w)$, we can choose $\varepsilon \in(0,1)$ such that $M^{\left(\frac{1}{1-\varepsilon}\right)}$ is also bounded on $\mathcal{M}_{q}^{p}(d x, w)$ by Lemma 2.9. Then we have

$$
\begin{aligned}
\mathrm{II} & \leq \sum_{l=1}^{\infty} \frac{C}{\left|2^{l} Q\right|} \int_{2^{l} Q}\left|b(y)-b_{Q}\right||f(y)| d y \\
& \leq C \sum_{l=1}^{\infty}\left(\frac{1}{\left|2^{l} Q\right|} \int_{2^{l} Q}\left|b(y)-b_{Q^{\mid}}\right|^{\frac{1}{\varepsilon}} d y\right)^{\varepsilon}\left(\frac{1}{\left|2^{l} Q\right|} \int_{2^{l} Q}|f(y)|^{\frac{1}{1-\varepsilon}} d y\right)^{1-\varepsilon} \\
& \leq C \sum_{l=1}^{\infty}\left(\frac{1}{\left|2^{l} Q\right|} \int_{2^{l} Q}\left|b(y)-b_{Q}\right|^{\frac{1}{\varepsilon}} d y\right)^{\varepsilon} \frac{\left\|\chi_{2^{l} Q}\right\|_{\mathcal{M}_{q}^{p}(d x, w)}}{\Phi_{p, q, w}\left(2^{l} Q\right)} \inf _{x \in 2^{l} Q} M^{\left(\frac{1}{1-\varepsilon}\right)} f(x) \\
& \leq C \sum_{l=1}^{\infty}\left(\frac{1}{\left|2^{l} Q\right|} \int_{2^{l} Q}\left|b(y)-b_{Q}\right|^{\frac{1}{\varepsilon}} d y\right)^{\varepsilon} \frac{\|f\|_{\mathcal{M}_{q}^{p}(d x, w)}}{\Phi_{p, q, w}\left(2^{l} Q\right)} .
\end{aligned}
$$

Now, we focus on the term related to the function $b$. It is easy to see that $\left|b_{2^{l} Q}-b_{Q}\right| \leq$ $C l\|b\|_{\text {Bмo. Hence, we have that }}$

$$
\begin{aligned}
\left(\frac{1}{\left|2^{l} Q\right|} \int_{2^{l} Q}\left|b(y)-b_{Q}\right|^{\frac{1}{\varepsilon}} d y\right)^{\varepsilon} & \leq\left(\frac{1}{\left|2^{l} Q\right|} \int_{2^{l} Q}\left|b(y)-b_{2^{l} Q}\right|^{\frac{1}{\varepsilon}} d y\right)^{\varepsilon}+\left|b_{2^{l} Q}-b_{Q}\right| \\
& \leq C(1+l)\|b\|_{\text {BMO }}
\end{aligned}
$$

for all $l \in \mathbb{N}$. It thus follows from Lemma 2.5 that

$$
\mathrm{II} \leq C\|b\|_{\mathrm{BMO}}\|f\|_{\mathcal{M}_{q}^{p}(d x, w)} \sum_{l=1}^{\infty} \frac{l}{\Phi_{p, q, w}\left(2^{l} Q\right)} \leq C\|b\|_{\mathrm{BMO}} \frac{\|f\|_{\mathcal{M}_{q}^{p}(d x, w)}}{\Phi_{p, q, w}(Q)}
$$

Next, we focus on the first term I. We employ (1.5), (1.7) and the weighted integral condition (1.3) for $p, q$ and $w$ to obtain that

$$
\begin{aligned}
I & \leq C\left|b(x)-b_{Q}\right|\left(\sum_{l=1}^{\infty} \frac{1}{\left|2^{l} Q\right|} \int_{2^{l} Q}|f(y)| d y\right) \\
& \leq C\left|b(x)-b_{Q}\right|\left(\sum_{l=1}^{\infty} \frac{1}{\left|2^{l} Q\right|}\left\|w^{-\frac{1}{q}} \chi_{2^{l} Q}\right\|_{H^{q^{\prime}, n(1-q / p)}}\|f\|_{\mathcal{M}_{q}^{p}(d x, w)}\right) \\
& \leq C\left|b(x)-b_{Q}\right| \cdot\|f\|_{\mathcal{M}_{q}^{p}(d x, w)} \sum_{l=1}^{\infty} \frac{1}{\Phi_{p, q, w}\left(2^{l} Q\right)} \\
& \leq C\left|b(x)-b_{Q}\right| \frac{\|f\|_{\mathcal{M}_{q}^{p}(d x, w)}}{\Phi_{p, q, w}(Q)} .
\end{aligned}
$$

Hence, we complete the proof of Lemma 4.5.

Additionally, we need the following simple observation. 
lm-160115-4 Lemma 4.6. Let $0<q<\infty, w \in A_{\infty}$ and $b \in$ BMO. Then for any cube $Q$, we have that

$$
\left(\frac{1}{w(Q)} \int_{Q}\left|b(x)-b_{Q}\right|^{q} w(x) d x\right)^{\frac{1}{q}} \leq C\|b\|_{\mathrm{BMO}}
$$

For the proof of Lemma 4.6, see [7] for example. Using Theorem 1.12 and Lemmas 4.5 and 4.6. let us complete the proof of Theorem 4.4.

Proof of Theorem 4.4. Fix any $f \in \mathcal{M}_{q}^{p}(d x, w)$ and any cube $Q$ and set

$$
\begin{aligned}
\mathrm{I} & :=|Q|^{\frac{1}{p}-\frac{1}{q}}\left(\int_{Q}\left|[b, T]_{0}\left(f \cdot \chi_{2 Q}\right)(x)\right|^{q} w(x) d x\right)^{\frac{1}{q}}, \\
\mathrm{II} & :=|Q|^{\frac{1}{p}-\frac{1}{q}}\left(\int_{Q}\left|\int_{\mathbb{R}^{n} \backslash 2 Q}(b(x)-b(y)) K(x, y) f(y) d y\right|^{q} w(x) d x\right)^{\frac{1}{q}} .
\end{aligned}
$$

Here, $[b, T]_{0}$ denotes the commutator in the sense of a bounded linear operator on $L^{\frac{1}{1-\varepsilon}}\left(\mathbb{R}^{n}\right)$ as before. All we have to do is to show that I, II $\leq C\|f\|_{\mathcal{M}_{q}^{p}(d x, w)}$. For the first term, we notice that $[b, T]_{0}\left(f \cdot \chi_{2 Q}\right) \in L^{\frac{1}{1-\varepsilon}}\left(\mathbb{R}^{n}\right)$, particularly,

$$
\lim _{l \rightarrow \infty} m_{[b, T]_{0}\left(f \cdot \chi_{2 Q}\right)}\left(2^{l} Q\right)=0
$$

holds for all $Q \in \mathcal{Q}$ and all medians. Hence, we may apply Theorem 1.12 to obtain that

$$
\mathrm{I} \leq\left\|[b, T]_{0}\left(f \cdot \chi_{2 Q}\right)\right\|_{\mathcal{M}_{q}^{p}(d x, w)} \leq C\left\|\left\{[b, T]_{0}\left(f \cdot \chi_{2 Q}\right)\right\}^{\sharp}\right\|_{\mathcal{M}_{q}^{p}(d x, w)} .
$$

Since we have the pointwise estimate; see [7, Lemma 3.5.5]:

$$
\left\{[b, T]_{0}\left(f \cdot \chi_{2 Q}\right)\right\}^{\sharp}(x) \leq C\|b\|_{\mathrm{BMO}}\left(M^{(\eta)}\left[f \cdot \chi_{2 Q}\right](x)+M^{2}\left[f \cdot \chi_{2 Q}\right](x)\right)
$$

for any $\eta>1$, by taking $\eta:=\frac{1}{1-\varepsilon}>1$, it follows that

$$
\mathrm{I} \leq C\|b\|_{\mathrm{BMO}}\|f\|_{\mathcal{M}_{q}^{p}(d x, w)} .
$$

Here, we also used the boundedness of $M^{\left(\frac{1}{1-\varepsilon}\right)}$ on $\mathcal{M}_{q}^{p}(d x, w)$.

For the second term, we employ Lemma 4.5 to obtain that

$$
\begin{aligned}
\mathrm{II} & \leq C\left(\|b\|_{\mathrm{BMO}}\|f\|_{\mathcal{M}_{q}^{p}(d x, w)}+\frac{\|f\|_{\mathcal{M}_{q}^{p}(d x, w)}}{\Phi_{p, q, w}(Q)}|Q|^{\frac{1}{p}-\frac{1}{q}}\left(\int_{Q}\left|b(x)-b_{Q}\right|^{q} w(x) d x\right)^{\frac{1}{q}}\right) \\
& =C\left(\|b\|_{\mathrm{BMO}}\|f\|_{\mathcal{M}_{q}^{p}(d x, w)}+\|f\|_{\mathcal{M}_{q}^{p}(d x, w)}\left(\frac{1}{w(Q)} \int_{Q}\left|b(x)-b_{Q}\right|^{q} w(x) d x\right)^{\frac{1}{q}}\right) .
\end{aligned}
$$

By virtue of Lemma 4.6, we see that II $\leq C\|b\|_{\text {BMO }}\|f\|_{\mathcal{M}_{q}^{p}(d x, w)}$.

Here, we recall a result of the commutators on weighted Morrey spaces $\mathcal{M}_{q}^{p}(w, w)$ of KomoriShirai type to compare this result with our results.

Theorem 4.7 ([15]). Let $1<q \leq p<\infty, w \in A_{q}$.

(1) Any singular integral operator $T$ is bounded on $\mathcal{M}_{q}^{p}(w, w)$.

(2) Assume that $b \in \mathrm{BMO}$. Then the commutator $[b, T]$ generated by a singular integral operator $T$ with respect to $b$ is bounded on $\mathcal{M}_{q}^{p}(w, w)$. 
As another application of Theorem 1.11 we consider the boundedness of $M$ on the Köthe dual space of $\mathcal{M}_{q}^{p}(d x, w)$. In [17, Lerner connected the boundedness of maximal operator $M$ and the sharp maximal inequality in the framework of Banach function spaces. Here, we do not give the definitions of the Banach function space $X$ and its Köthe dual space $X^{\prime}$. We refer [2] for these definitions.

lerner Proposition 4.8. 17, Corollary 4.3] Let $X$ be a Banach function space on which $M$ is bounded. Then $M$ is bounded on $X^{\prime}$ if and only if there exists $c>0$ such that for any $f \in L^{0}\left(\mathbb{R}^{n}\right)$ such that $\lim _{R \rightarrow \infty} f^{*}(R)=0$,

$$
\|f\|_{X} \leq c\left\|f^{\sharp}\right\|_{X} .
$$

Unfortunately, the Morrey spaces are not Banach function spaces in general; see 28, Example 3.3]. Nevertheless, we still have an analogy of Proposition 4.8 in the framework of "ball Banach function spaces". We refer [28] for the motivation of the notion of ball Banach function spaces and its definitions. We may define the Köthe dual space $X^{\prime}$ of the ball Banach function spaces $X$ by the same way as the one of the Banach function space. We also note that $\mathcal{M}_{q}^{p}(d x, w)$ is the ball Banach function spaces as long as $M$ is bounded on $\mathcal{M}_{q}^{p}(d x, w)$.

By a similar argument as in [17, We obtain the Proposition 4.8 for the ball Banach function spaces version.

pr-151209-1 Proposition 4.9. Let $X$ be a ball Banach function space on which $M$ is bounded. Then $M$ is bounded on $X^{\prime}$ if and only if there exists $c>0$ such that for any $f \in L^{0}\left(\mathbb{R}^{n}\right)$ satisfying

$$
\lim _{R \rightarrow \infty} f^{*}(R)=0,
$$

it holds that

$$
\|f\|_{X} \leq c\left\|f^{\sharp}\right\|_{X} .
$$

rm-151209-1 Remark 4.10. The assumption (4.5) in Proposition 4.9 implies (1.18) in Theorem 1.12. In fact, if one assumes (4.5), then we have that

$$
\left|m_{f}\left(2^{l} Q\right)\right| \leq\left(f \cdot \chi_{2^{l} Q}\right)^{*}\left(2^{-2}\left|2^{l} Q\right|\right) \leq f^{*}\left(2^{-2}\left|2^{l} Q\right|\right) \rightarrow 0 .
$$

As the example of $f$, where $f: \mathbb{R} \rightarrow \mathbb{R}$ is given by,

$$
f=\sum_{j=3}^{\infty} \chi_{[j !, j !+1]}
$$

shows that the assumption (4.5) is stronger than (1.18).

Thanks to Proposition 4.9 and Remark 4.10 the inequality 4.6) for any $f \in L^{0}\left(\mathbb{R}^{n}\right)$ satisfying (1.18) implies the boundedness of $M$ on $X^{\prime}$. Particularly, we proved (4.6) with $X=\mathcal{M}_{q}^{p}(d x, w)$ under the suitable conditions in Corollary 1.14. On the other hand, the second author and Tanaka obtained the characterization of the Köthe dual space of $\mathcal{M}_{q}^{p}(d x, w)$ in 27, 28]. For $\sigma(x)=w(x)^{-\frac{q^{\prime}}{q}}$, we denote all measurable functions $f$ for which norm

$$
\|f\|_{H^{q^{\prime}, n(1-q / p)}(d x, \sigma)}:=\left\|w^{-\frac{1}{q}} f\right\|_{H^{q^{\prime}, n(1-q / p)}}
$$

is finite by $H^{q^{\prime}, n(1-q / p)}(d x, \sigma)$. Then we have that $\mathcal{M}_{q}^{p}(d x, w)^{\prime}=H^{q^{\prime}, n(1-q / p)}(d x, \sigma)$ when $1<q \leq p<\infty$; see [27, 28. As a result, we obtain the boundedness of $M$ on the Köthe dual space $H^{q^{\prime}, n(1-q / p)}(d x, \sigma)$ under the certain conditions:

th-160117-1 Theorem 4.11. Let $1<q \leq p<\infty$ and assume the boundedness of $M$ on $\mathcal{M}_{q}^{p}(d x, w)$ and the weighted integral condition (1.3) for $p, q$ and $w$. Then $M$ is also bounded on $H^{q^{\prime}, n(1-q / p)}(d x, \sigma)$ with $\sigma(x)=w(x)^{-\frac{q^{\prime}}{q}}$ :

$$
\|M f\|_{H^{q^{\prime}, n(1-q / p)}(d x, \sigma)} \leq C\|f\|_{H^{q^{\prime}, n(1-q / p)}(d x, \sigma)} \quad\left(f \in H^{q^{\prime}, n(1-q / p)}(d x, \sigma)\right) .
$$


Rerated to Theorem 4.11, Ho 9] characterized the BMO norm in the context of general function space including Lebesgue spaces. Let us overview the Ho's result. Given a Banach function space $X$ equipped with a norm $\|\cdot\|_{X}$, we define the generalized BMO norm

$$
\|b\|_{\mathrm{BMO}_{X}}:=\sup _{Q \in \mathcal{Q}} \frac{1}{\left\|\chi_{Q}\right\|_{X}}\left\|\left(b-b_{Q}\right) \chi_{Q}\right\|_{X} .
$$

In 9], Ho proved that if $M$ is bounded on the associate space $X^{\prime}$, then $\|b\|_{\mathrm{BMO}_{X}}$ and $\|b\|_{\mathrm{BMO}}$ are equivalent.

We can generalize Ho's result [9] to the ball Banach function spaces as before. Hence, by combining Theorem 4.11 with the idea of Ho, we obtain the following:

Corollary 4.12. Let $1<q \leq p<\infty$ and assume the boundedness of $M$ on $\mathcal{M}_{q}^{p}(d x, w)$ and the weighted integral condition (1.3) for $p, q$ and $w$. Define a generalized BMO norm with respect to the weighted Morrey space of Samko's type

$$
\|b\|_{\mathrm{BMO}_{\mathcal{M}_{q}^{p}(d x, w)}}:=\sup _{Q \in \mathcal{Q}} \frac{1}{\Phi_{p, q, w}(Q)}\left\|\left(b-b_{Q}\right) \chi_{Q}\right\|_{\mathcal{M}_{q}^{p}(d x, w)} .
$$

Then the norm equivalence

$$
\|b\|_{\mathrm{BMO}_{\mathcal{M}_{q}^{p}(d x, w)}} \sim\|b\|_{\mathrm{BMO}}
$$

holds for all $b \in \mathrm{BMO}$.

\section{REFERENCES}

[1] D. R. Adams, J. Xiao, Morrey spaces in harmonic analysis, Ark. Mat., 50 (2012), no. 2, 201-230.

[2] C. Bennett, R. Sharpley, Interpolation of Operators, Academic Press, 1988.

[3] V. I. Burenkov, V. S. Guliyev, A. Serbetci and T. V. Tararykova, Necessary and sufficient condition for the boundedness of genuine singular integral operators in local Morrey-Type spaces, Eurasian Math. J. 1 , no. $1,32-53,2010$.

[4] R. R. Coifman and C. Fefferman, Weighted norm inequalities for maximal functions and singular integrals, Studia Math., 51 241-250, 1974

[5] N. Fujii, A condition for a two-weight norm inequality for singular integral operators, Studia Math., 98 (3) (1991), 175-190.

[6] J. Garcia-Cuerva, J. L. Rubio de Francia, Weighted Norm Inequalities and Related Topics, North-Holland, Math. Stud., 116 (1985).

[7] L. Grafakos, Moderen Fourier Analysis, Third Edition, Springer, GTM250.

[8] D. I. Hakim, E. Nakai and Y. Sawano, Generalized fractional maximal operators and vector-valued inequalities on generalized Orlicz-Morrey spaces, Revista Matemática Complutense, (2015), 1-32.

[9] K.-P. Ho, Atomic decomposition of Hardy spaces and characterization of BMO via Banach function spaces, Anal. Math. 38 (2012), 173-185.

[10] T. Hytönen, Weighted norm inequalities, Lecture notes of a course at the University of Helsinki, Winter 2011, http://wiki-app.it.helsinki.fi/download/attachments/64424417/weighted.pdf

[11] T. Hytönen, The sharp weighted bound for general Calderón-Zygmund operators, Ann. Math. 175(3), (2012), 1473-1506.

[12] B. Jawerth and A. Torchinsky, Local sharp maximal functions, J. Approx. Theory, 43 (1965), 231-270.

[13] F. John, Quasi-isometric mappings, Seminari 1962-1963 di Analisi, Algebra, Geometria e Topologia, Ist Nazarene Alta Matematica 2 (Edizioni Cremonese, Rome, 1965), 462-473.

[14] Y. Komori-Furuya, Local Good- $\lambda$ Estimate for the Sharp Maximal Function and Weighted Morrey Space, Journal of Function Spaces Volume 2015 (2015), Article ID 651825, 4 pages http://dx.doi.org/10.1155/2015/651825

[15] Y. Komori and S. Shirai, Weighted Morrey spaces and a singular integral operator, Math. Nachr. 282 (2009), no. 2, 219-231.

[16] A.K. Lerner, On weighted estimates of non-increasing rearrangements, East J. Approx. 4 (1998), 277-290.

[17] A.K. Lerner, Some remarks on the Fefferman-Stein inequality, J. Anal. Math. 112 (2010), 329-349.

[18] A.K. Lerner, On an estimate of Calderön-Zygmund operators by dyadic positive operators, J. Anal. Math. 121 (2013), 141-161.

[19] A.K. Lerner, S. Ombrosi, A boundedness criterion for general maximal operators, Publ. Mat., 54 (2010) no. $1,53-71$. 
[20] B. Muckenhoupt, R. L. Wheeden, Weighted bounded mean oscillation and the Hilbert transform, Studia Math., 54 (1976), 221-237.

[21] E. Nakai, Hardy-Littlewood maximal operator, singular integral operators and the Riesz potentials on generalized Morrey spaces, Math. Nachr. 166 (1994), 95-103.

[22] S. Nakamura, Generalized weighted Morrey spaces and classical operators, Math. Nachr., online.

[23] S. Nakamura, T. Noi and Y. Sawano, Generalized Morrey spaces and trace operator, Sci. China Math. 59 (2016), no. 2, 281-336.

[24] N Samko, Weighted Hardy and singular operators in Morrey spaces, J. Math. Anal. Appl. 350 (2009), $56-72$.

[25] C. Pérez, Endpoint estimates for commutators of singular integral operators, J. Funct. Anal., 128 (1995), 163-185.

[26] Y. Sawano and H. Tanaka, Sharp maximal inequalities and commutators on Morrey spaces with nondoubling measures, Taiwanese J. Math. 11 (2007), no. 4, 1091-1112.

[27] Y. Sawano and H. Tanaka, Fatou property of predual Morrey spaces with non-doubling measures, Int. J. Appl. Math. 27 (2014), no. 3, 283-296.

[28] Y. Sawano, H. Tanaka, The Fatou Property of Block spaces, J. Math. Sci. Univ. Tokyo, 22 (2015), 663-683.

[29] E.M. Stein, Harmonic Analysis: Real-Variable Methods, Orthogonality, and Oscillatory Integrals, Princeton Univ. Press, 1993.

[30] H. Tanaka, Two-weight norm inequalities on Morrey spaces, Ann. Acad. Sci. Fenn. Math. 40 (2015), $773-791$.

[31] J.O. Strömberg, Bounded mean oscillation with Orlicz norms and duality of Hardy spaces, Indiana Univ. Math. J. 28 (1979), 511-544. 